\title{
AN OPTICAL SURVEY OF THE PARTIALLY EMBEDDED YOUNG CLUSTER IN NGC 7129
}

\author{
S. E. Dahm ${ }^{1}$ and L. A. Hillenbrand ${ }^{2}$ \\ ${ }^{1}$ W. M. Keck Observatory, Kamuela, HI 96743, USA; sdahm@keck.hawaii.edu \\ ${ }^{2}$ California Institute of Technology, USA \\ Received 2015 February 12; accepted 2015 March 19; published 2015 May 27
}

\begin{abstract}
NGC 7129 is a bright reflection nebula located in the molecular cloud complex near $l=105: 4, b=+9: 9$, about $1.15 \mathrm{kpc}$ distant. Embedded within the reflection nebula is a young cluster dominated by a compact grouping of four early-type stars: $\mathrm{BD}+65^{\circ} 1638(\mathrm{~B} 3 \mathrm{~V}), \mathrm{BD}+65^{\circ} 1637$ (B3e), SVS 13 (B5e), and $\mathrm{LkH} \alpha 234$ (B8e). About 80 $\mathrm{H} \alpha$ emission sources brighter than $V \sim 23$ are identified in the region, many of which are presumably $\mathrm{T}$ Tauri star members of the cluster. We also present deep $(V \sim 23)$, optical $\left(V R_{C} I_{C}\right)$ photometry of a field centered on the reflection nebula and spectral types for more than 130 sources determined from low dispersion, optical spectroscopy. The narrow pre-main sequence evident in the color-magnitude diagram suggests that star formation was rapid and coeval. A median age of about $1.8 \mathrm{Myr}$ is inferred for the $\mathrm{H} \alpha$ and literature-identified X-ray emission sources having established spectral types, using pre-main sequence evolutionary models. Our interpretation of the structure of the molecular cloud and the distribution of young stellar objects is that $\mathrm{BD}+65^{\circ} 1638$ is primarily responsible for evacuating the blister-like cavity within the molecular cloud. $\mathrm{LkH} \alpha 234$ and several embedded sources evident in near-infrared adaptive optics imaging have formed recently within the ridge of compressed molecular gas. The compact cluster of low-mass stars formed concurrently with the early-type members, concentrated within a central radius of $\sim 0.7 \mathrm{pc}$. Star formation is simultaneously occurring in a semi-circular arc some $\sim 3 \mathrm{pc}$ in radius that outlines remaining dense regions of molecular gas. High dispersion, optical spectra are presented for $\mathrm{BD}+65^{\circ} 1638, \mathrm{BD}+65^{\circ} 1637$, SVS $13, \mathrm{LkH} \alpha 234$, and V350 Cep. These spectra are discussed in the context of the circumstellar environments inferred for these stars.
\end{abstract}

Key words: open clusters and associations: individual (NGC 7129) - stars: formation - stars: pre-main sequence

Supporting material: machine-readable and VO table

\section{INTRODUCTION}

The bright reflection nebula NGC $7129\left(l=105^{\circ}, b=\right.$ $+9 \circ 9)$ is illuminated by the early-type members of a young stellar cluster emerging from a molecular cloud complex composed of the dark nebulae Lynds 1181 (L1181), L1183, and TDS 395 (Taylor et al. 1987; Yonekura et al. 1997). Signatures of star formation activity are present throughout the region including two dozen Herbig-Haro $(\mathrm{HH})$ objects, two large molecular outflows, numerous nebulous filaments, and embedded infrared sources. The cluster is dominated by a tight grouping of B-type stars that includes $\mathrm{BD}+65^{\circ} 1638$ (B3), BD $+65^{\circ} 1637$ (B3e), SVS 13 (B5e), and the Herbig Be star LkH $\alpha$ 234 (B8e). An elongated cavity one parsec in diameter has been carved out of L1181, presumably by the stellar winds and ultraviolet flux emanating from the central grouping of B-stars. Shown in Figure 1 is a three-color $\left(V R_{C} I_{C}\right.$-band) image of the reflection nebula obtained by G. H. Herbig in 1999 October using the University of Hawaii (UH) $2.2 \mathrm{~m}$ telescope on Mauna Kea. The image clearly depicts the dense molecular ridge to the east and south, the evacuated region around the four luminous central stars, and the emerging young cluster lying within the reflection nebula. The distance of NGC 7129 was estimated to be $\sim 1 \mathrm{kpc}$ by Racine (1968), but more recent estimates include $1.26 \mathrm{kpc}$ (Shevchenko \& Yakubov 1989) and $1.15 \mathrm{kpc}$ (Straižys et al. 2014), which is adopted in this analysis. Kun et al. (2008) present a comprehensive literature review of the Cepheus star-forming region including NGC 7129 and its embedded cluster. Below we summarize only pertinent studies that are directly relevant to the present survey of the young stellar population.
NGC 7129 and its associated molecular clouds have been studied extensively at millimeter wavelengths by Loren (1975, 1977), Bechis et al. (1978), Bertout (1987), and Miskolczi et al. (2001). These ${ }^{12} \mathrm{CO}$ and ${ }^{13} \mathrm{CO}$ surveys have identified multiple density enhancements in the region, a semi-circular ridge of $\mathrm{CO}$ emission to the north, east, and south of the reflection nebula, and the large evacuated cavity centered near $\mathrm{BD}+65^{\circ} 1637$. In the infrared, Bechis et al. (1978) detected two $100 \mu \mathrm{m}$ sources in the area: $\mathrm{LkH} \alpha 234$ and far-infrared source 2 (FIRS 2), which has no optical counterpart, located some $2^{\prime}$ south of BD $+65^{\circ} 1638$. At least two molecular outflows have been identified in the region (Bechis et al. 1978; Edwards \& Snell 1983; Liseau \& Sandell 1983); one centered on FIRS 2 and the other originating near $\mathrm{LkH} \alpha 234$. Yonekura et al. (1997) estimate the total mass of the molecular cloud group associated with NGC 7129 to be $\sim 2900 M_{\odot}$.

A single-channel $2.2 \mu \mathrm{m}$ survey of the region was carried out by Strom et al. (1976), hereafter SVS, in a search for an embedded population of newly formed stars. Although none were identified, the detection limit of the survey was marginally close to the expected brightness of an average T Tauri star. Cohen \& Schwartz (1983) identified four new infrared sources in the region, one of which was V350 Cep, an M2-type T Tauri star that brightened dramatically between 1970 and 1976. Ibryamov et al. (2014) present a photometric study for this source that includes a long-term light curve constructed from archival data.

Gutermuth et al. (2004) used the 2MASS, the FLAMINGOS spectrograph on the Multiple Mirror Telescope, and the Infrared Array Camera (IRAC) on board the Spitzer Space Telescope to complete an extensive $1.2-8.0 \mu \mathrm{m}$ survey of the cluster, ultimately determining a disk fraction of $54 \% \pm 14 \%$ 


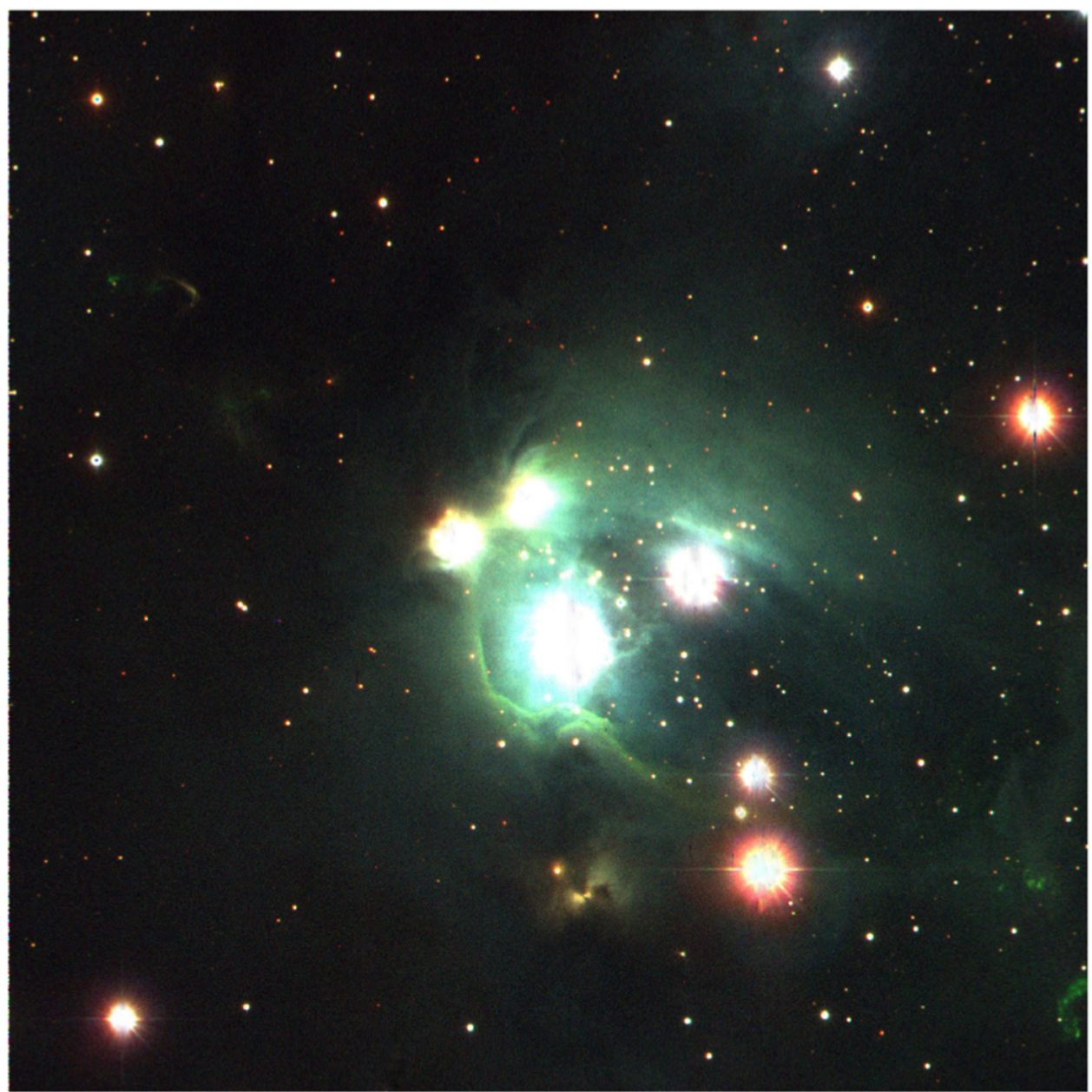

Figure 1. $V R_{C} I_{C}$-band three-color image of NGC 7129 and the L1181 molecular cloud obtained by G. H. Herbig in 1999 October (orientation: north up, east left). This $7.5 \times 7.5$ field is centered on the grouping of early-type stars within the reflection nebula that includes BD+65 1638 (south), $\mathrm{BD}+65^{\circ} 1637$ (west), LkH $\alpha 234$ (east) and SVS 13 (north). LkH $\alpha 234$ lies within the dense ridge of molecular gas that extends several arcminutes and outlines the evacuated region of the molecular cloud. To the northeast, just over half the distance from the cluster center to the corner, is the ghostly outline of HH 105, and to the southwest, near the edge of the field of view, HH 103 which is likely associated with FIRS 2 or V392 Cep (RNO 138). FIRS 2, undetected at optical wavelengths, lies near the center of the bottom edge of the field of view, just south of V392 Cep.

within the cluster core (defined as a $0.5 \mathrm{pc}$ radius arc extending from the peak of the local stellar surface density). From a star count analysis, Gutermuth et al. (2004) estimate that there are about 80 cluster members within the core, down to a limiting magnitude of $K_{S}=16 \mathrm{mag}$, or approximately $0.055 M_{\odot}$ for unattenuated sources. In the peripheral areas Gutermuth et al. (2004) estimate that the extended cluster population is roughly equal to that of the core.

Muzerolle et al. (2004) present $24 \mu \mathrm{m}$ Multi-band Imaging Photometer for Spitzer (MIPS) imaging of NGC 7129 and provide a census of the cluster population outside of the reflection nebula. Of the 36 sources detected at $24 \mu \mathrm{m}$ and in at least three IRAC passbands, 13 were classified as Class 0/I protostars with envelopes including FIRS 2 (Class 0), another 18 as Class II sources (classical T Tauri stars, CTTS) with relatively low infrared luminosity, and six were classified as Class III sources with emission consistent with that of pure stellar photospheres. The majority of these sources are located in an arc sweeping north-east to south of the reflection nebula, effectively tracing the densest regions of the remnant molecular cloud. Muzerolle et al. (2004) conclude that star formation is not concentrated in the cluster core, but rather dispersed over a substantial area, some $\sim 3 \mathrm{pc}$ in scale.
Stelzer \& Scholz (2009) combined the mid-infrared Spitzer surveys of Gutermuth et al. (2004) and Muzerolle et al. (2004) with a shallow $22 \mathrm{ks}$ Chandra ACIS X-ray observation of the region. From the Spitzer infrared excess sources and the 59 Chandra X-ray detections, they define a sample of 26 Class II and 25 Class III cluster members, estimated to be complete to $\sim 0.5 M_{\odot}$. The disk fraction of the least biased sub-sample composed of lightly extincted sources is $\sim 33_{-19}^{+24} \%$.

While NGC 7129 has been studied extensively at millimeter, micron, and X-ray wavelengths, it remains poorly characterized in the optical. Hartigan \& Lada (1985) obtained $V R_{C} I_{C}$ and narrowband $\mathrm{H} \alpha$ CCD imaging of two regions in NGC 7129 which include most sources from the infrared surveys of Cohen \& Schwartz (1983) and SVS. $V R_{C} I_{C}$ photometry is provided for about 30 sources in these two fields. Magakian et al. (2004) identified $22 \mathrm{H} \alpha$ emission stars in the central and northeast cluster regions, complete to $V \sim 20$ using slitless spectroscopy. $V R I$-band photometry for about 100 sources was also obtained, but not provided in the published analysis. Magakian et al. (2004) concluded that most of the detected $\mathrm{H} \alpha$ emission sources were $\mathrm{T}$ Tauri stars. Vilnius seven-color photometry down to $V=18.8$ is provided by Straižys et al. (2014) for 159 


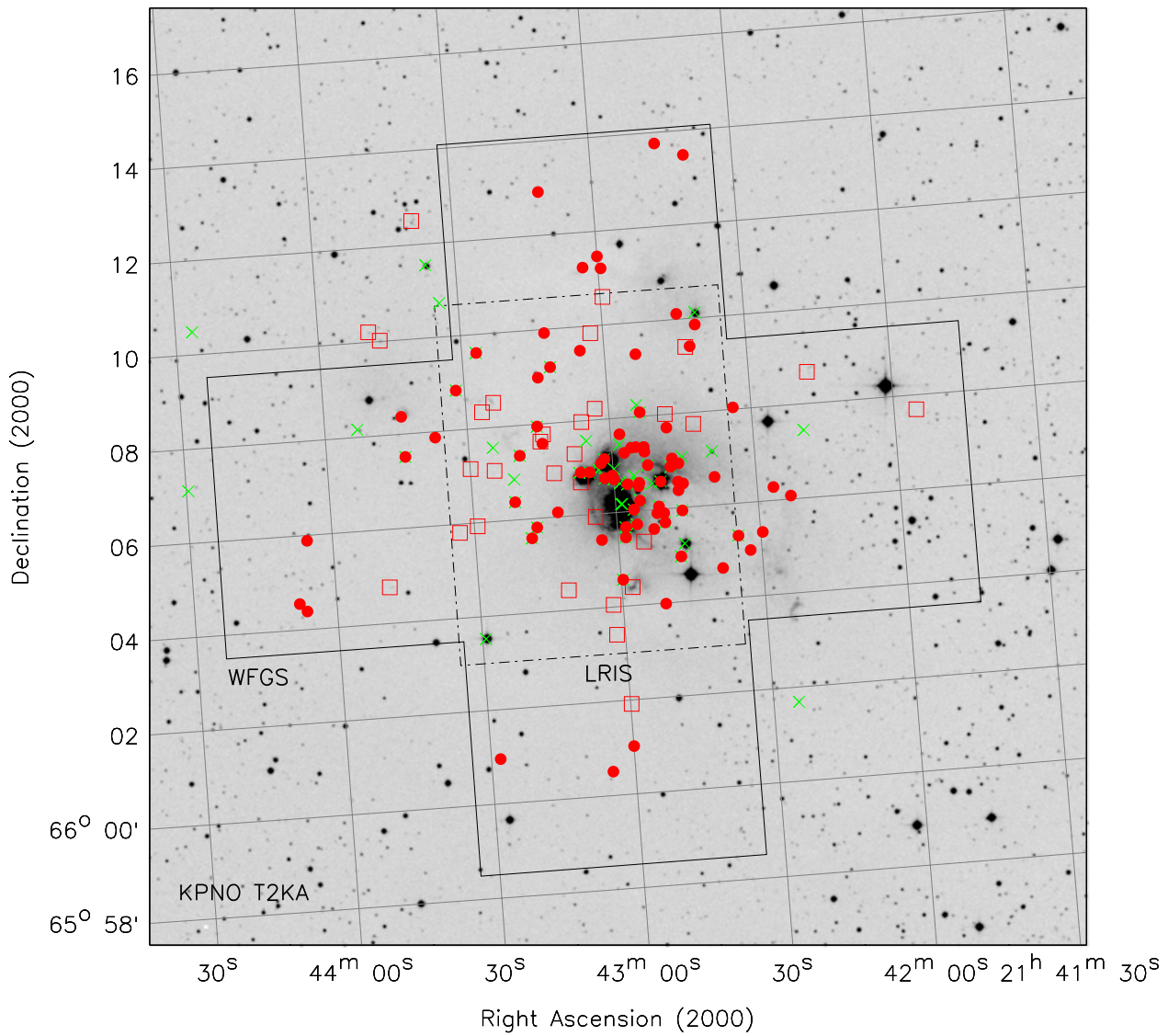

Figure 2. $20^{\prime} \times 20^{\prime}$ field of the red DSS image of NGC 7129. The $150 \operatorname{arcmin}^{2}$ area surveyed with the WFGS and the region imaged with LRIS are outlined. The KPNO T2KA imaging data encompasses the entire field of view. Filled red circles mark the positions of $\mathrm{H} \alpha$ emission sources, green crosses represent X-ray detected sources from the Chandra ACIS observation, and open red squares show the positions of Class 0/I/II sources that were not detected in X-rays by Stelzer \& Scholz (2009) or by the $\mathrm{H} \alpha$ emission survey presented in this work.

stars in the cluster region. The photometric data was used to classify about half of the detected sources, assigning spectral types, luminosity classes and deriving extinction estimates. The extinction across a $23^{\prime} \times 23^{\prime}$ area varies between 0.6 and $2.8 \mathrm{mag}$, but in the densest parts of the molecular cloud, $A_{V}$ approaches $\sim 13$ mag. Straižys et al. (2014) determine the age of the cluster to be between 0.2 and 3 Myr by plotting six earlytype cluster members on the HR diagram and using the evolutionary models of Palla (2005).

From the literature described above, some 90 pre-main sequence candidates have been identified in the cluster region spanning an area $\sim 10^{\prime}$ in diameter or $\sim 3.3 \mathrm{pc}$ in physical scale. These sources were detected using a number of youth discriminants including $\mathrm{H} \alpha$ emission (Hartigan \& Lada 1985; Magakian et al. 2004), X-ray emission (Stelzer \& Scholz 2009), and infrared excess (Gutermuth et al. 2004; Muzerolle et al. 2004; Stelzer \& Scholz 2009). No comprehensive optical photometric and spectroscopic census of the young cluster population, however, has been published to date.

In this paper we present deep $(V \sim 23) V R_{C} I_{C}$-band photometry for a field roughly centered on the reflection nebula. Results from a slitless grism, $\mathrm{H} \alpha$ spectroscopic survey of a $\sim 125 \mathrm{arcmin}^{2}$ area of the cluster are discussed. Spectral types are provided for $\sim 130$ stars in the region, determined from low-dispersion, multi-object spectroscopy. We also present high dispersion $(R \sim 45,000)$, optical spectra for $\mathrm{BD}+65^{\circ} 1638, \mathrm{BD}+65^{\circ} 1637, \mathrm{LkH} \alpha 234, \mathrm{SVS} 13$, and V350 Cep. Finally near-infrared, adaptive optics imaging of $\mathrm{LkH} \alpha$
234 is examined and compared with archived Spitzer IRAC data.

This paper is organized as follows: in Section 2 we discuss the observations made by the authors and by G. H. Herbig in support of this investigation. In Section 3 we present the highdispersion spectra of the early-type stars and of V350 Cep; in Section 4 the stellar population of NGC 7129 is examined to include the early-type stars, the $\mathrm{H} \alpha$ emission sources, and the Xray detected sources. We present in tabular form photometry and spectral types determined at optical wavelengths for two subsets of stars: (1) those exhibiting $\mathrm{H} \alpha$ and/or X-ray emission, and (2) others in the field, some of which exhibit infrared excess. In Section 5 we discuss the color-magnitude diagram of the premain sequence population, the average extinction suffered by those stars with assigned spectral types, the ages and masses of the cluster members, and the infrared color-color diagrams. In Section 6 we discuss the progression of star formation in the parent molecular clouds as well as the star-forming history in the region. We examine the source of the molecular outflow near $\mathrm{LkH} \alpha 234$ and the origin of the photo-dissociation region (PDR) enveloping the early-type stars.

\section{OBSERVATIONS}

\subsection{Optical Photometry}

Three epochs of optical imaging data were obtained of the NGC 7129 region between 1993 and 1999. Extinction corrections and transformation to the Landolt (1992) system were 

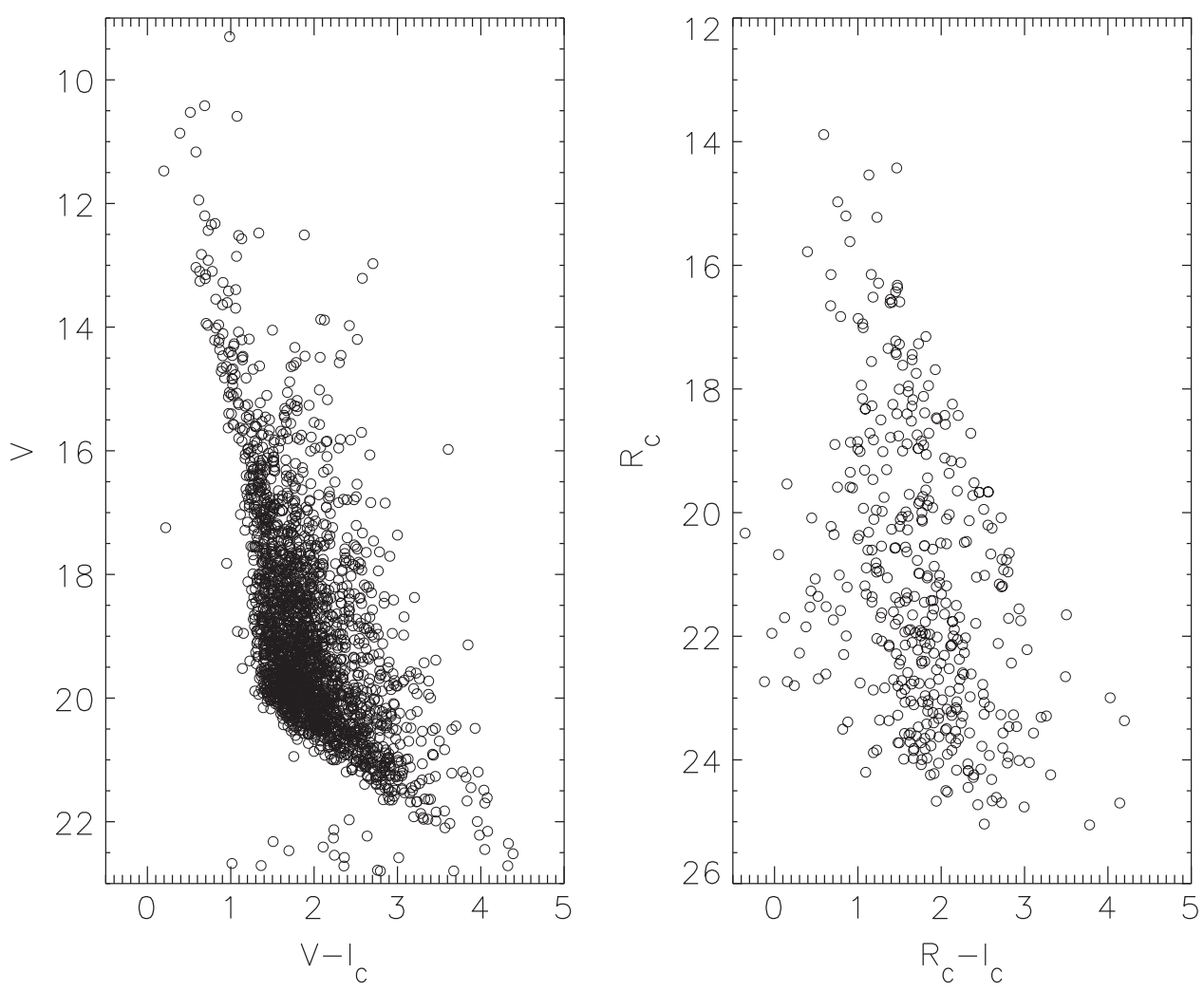

Figure 3. (Left panel) the observed $V-I_{C}, V$ color-magnitude diagram for all $\sim 2500$ sources detected in the KPNO 1993 CCD imaging survey of a $23^{\prime} \times 23^{\prime}$ region. (Right panel) the $R_{C}-I_{C}, R_{C}$ color-magnitude diagram for all sources detected in the Keck LRIS $8^{\prime} \times 6^{\prime}$ imaging survey of the core of NGC 7129 . No corrections for interstellar reddening have been applied to the photometry.

achieved for all three sets by observing several standard fields periodically throughout the night at various air masses. The photometric calibrations included zero point, airmass, and color terms except when indicated below.

The first and most extensive survey consisted of $V R_{C} I_{C}$-band CCD imaging obtained using the T2KA CCD at the KPNO $0.9 \mathrm{~m}$ telescope in 1993 June by LAH and S. Strom. The T2KA detector is a $2048 \times 2048 \mathrm{CCD}$ with $24 \mu \mathrm{m}$ pixels yielding a platescale of 0.69 pixel $^{-1}$. Twilight flats were obtained at the beginning and end of the nights in all filters to remove pixel to pixel variations in response. Exposure times were 3, 30, and $300 \mathrm{~s}$ in all passbands. The optical images span a region $23^{\prime} \times 23^{\prime}$ in area with a photometric completeness limit of $V \sim$ 18.5. The faintest sources evident on the CCD images are about three magnitudes fainter. The region imaged by the KPNO T2KA CCD encompasses the $20^{\prime} \times 20^{\prime}$ red DSS image of the region shown in Figure 2. The T2KA CCD images were reduced using standard tasks and procedures available in the Image Reduction and Analysis Facility (IRAF). Point-spread function (PSF) fitting photometry was performed using the DAOPHOT package of IRAF. The $V-I_{C}, V$ color-magnitude diagram for all $\sim 2500$ sources with photometry available from the T2KA CCD imaging is shown in Figure 3 (left panel). We provide a supplemental on-line catalog with the J2000 coordinates, optical $\left(V, V-R_{C}\right.$, and $\left.R_{C}-I_{C}\right)$ photometry, and photometric errors for all $\sim 2500$ sources.

The second epoch of $R_{C} I_{C}$-band photometry was obtained using the Low Resolution Imaging Spectrograph (LRIS) on Keck II in 1999 June by LAH. The $8^{\prime} \times 6^{\prime}$ LRIS images were centered on $\mathrm{LkH} \alpha 234$ (Figure 2) and reveal dozens of faint cluster members within the compact grouping of early-type stars. Exposure times ranged from 3 to $600 \mathrm{~s}$ to ensure that the majority of sources were unsaturated. Tasks within the PHOT package of IRAF were used to carry out aperture photometry. Photometric calibration was achieved using zero point and airmass terms only, i.e., no color terms were applied. Shown in Figure 3 (right panel) is the $R_{C}-I_{C}, R_{C}$ color-magnitude diagram for all sources in the LRIS field of view with no allowance being made for interstellar reddening.

Finally $B V R_{C} I_{C}$ photometry of the NGC 7129 region was obtained in 1999 October at the $f / 10$ focus of the UH $2.2 \mathrm{~m}$ telescope on Mauna Kea by G. H. Herbig. The detector was a Tektronix $2048 \times 2048 \mathrm{CCD}$ with $24 \mu \mathrm{m}$ pixels. The field was imaged in all filters during photometric conditions and in average seeing conditions for Mauna Kea, 0.75 . The plate scale was 0.22 pixel $^{-1}$, and the imaged field was approximately $7.5 \times 7.5$ in area. Three exposures were obtained in each filter with integration times of 5,60, and $300 \mathrm{~s}$. Image reduction and analysis were accomplished by spectral energy distribution (SED) using IRAF. Aperture and PSF fitting photometry were carried out with the PHOT, PSF, and ALLSTAR tasks in the DAOPHOT package of IRAF. Bright, isolated, single stars were selected across the field to construct a characteristic PSF for the images in each color.

Photometry from the three optical imaging surveys are compared in Figure 4, 1993 June (KPNO) versus 1999 October (UH $2.2 \mathrm{~m}$ ) and Figure 5, 1993 June (KPNO) versus 1999 June (Keck LRIS). There is relative agreement, however a slight offset $(\sim+0.16 \mathrm{mag})$ exists between the $V-I_{C}$ colors of the 1999 October UH $2.2 \mathrm{~m}$ observations and the 1993 June KPNO observations. This offset originates from extended red transmission from the UH $2.2 \mathrm{~m} I_{C}$ filter, which is documented by Courtois et al. (2011). Given the presence of this red leak in the UH $2.2 \mathrm{~m} I_{C}$ filter, the optical photometry presented here is 

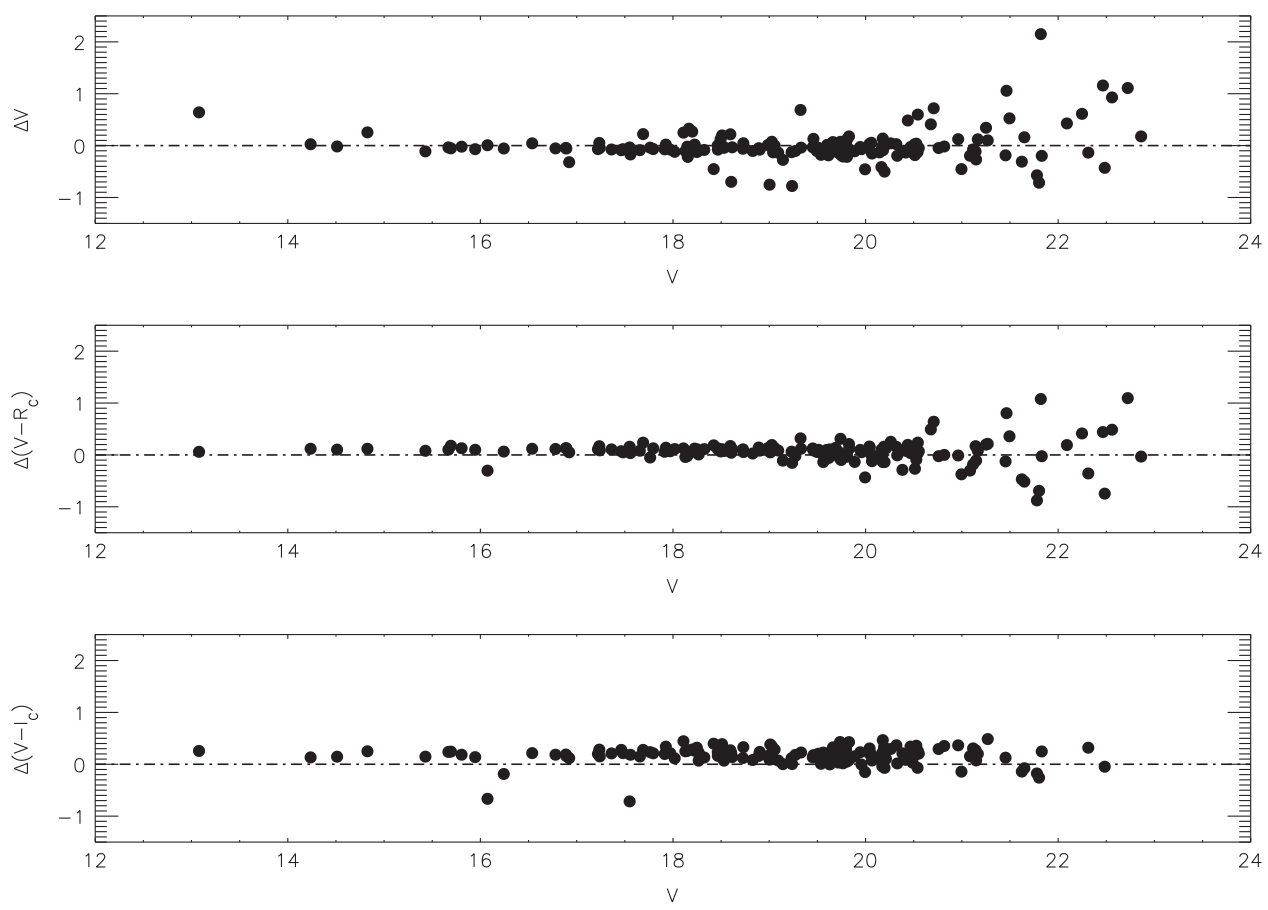

Figure 4. Comparison of the KPNO photometry from 1993 June with that from the UH $2.2 \mathrm{~m}$ from 1999 October. The slight offset present in the $V-I_{C}$ colors arises from extended red transmission in the UH $2.2 \mathrm{~m} \mathrm{I}_{C}$ filter.
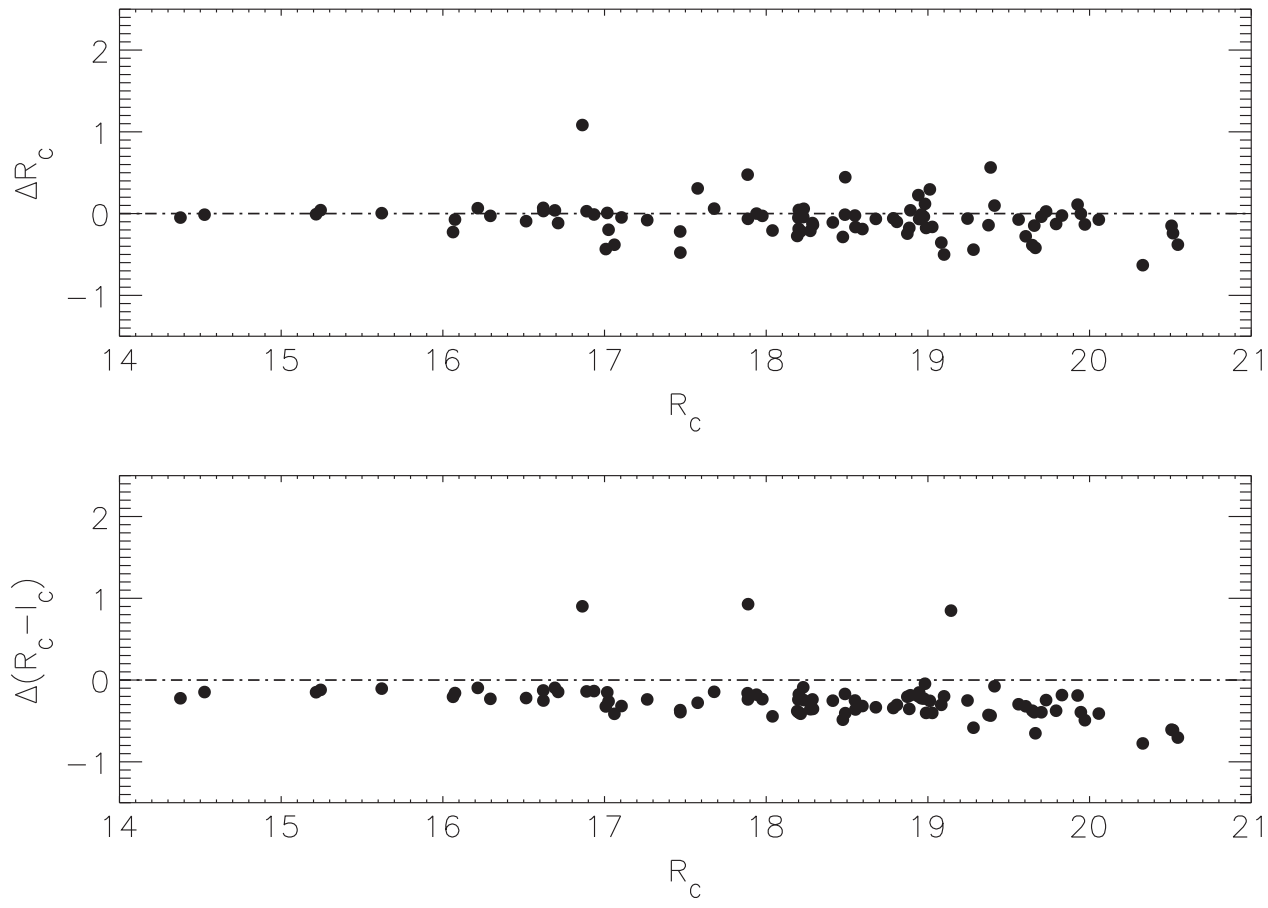

Figure 5. Comparison of the KPNO photometry from 1993 June with that from the Keck LRIS imaging survey of 1999 June. Photometric calibration of the LRIS data was achieved by applying airmass and zero point corrections only, i.e., no color terms were applied, which likely explains the color offset.

taken primarily from the 1993 KPNO observations, unless photometry for a given source were not available. In these circumstances, the photometry from the $1999 \mathrm{UH} 2.2 \mathrm{~m}$ observations were substituted with a -0.16 mag correction being applied to the $V-I_{C}$ color. Differences in the $R_{C}-I_{C}$ colors of the 1993 KPNO and the 1999 Keck LRIS data evident in Figure 5 likely arise from the missing color term in the photometric calibration of the latter data set.

\subsection{Ho Slitless Grism Spectroscopy}

The H $\alpha$ emission survey of NGC 7129 was carried out on 10 October 2003 by SED using the Wide-Field Grism Spectrograph (WFGS) installed at the $f / 10$ Cassegrain focus of the University of Hawaii $2.2 \mathrm{~m}$ telescope. A 420 line $\mathrm{mm}^{-1}$ grism blazed at $6400 \AA$ yielded a dispersion of $3.85 \AA$ pixel $^{-1}$. The narrowband $\mathrm{H} \alpha$ filter isolated a region of the first-order spectra between $\sim 6300$ and $6750 \AA$. The WFGS imaged upon 


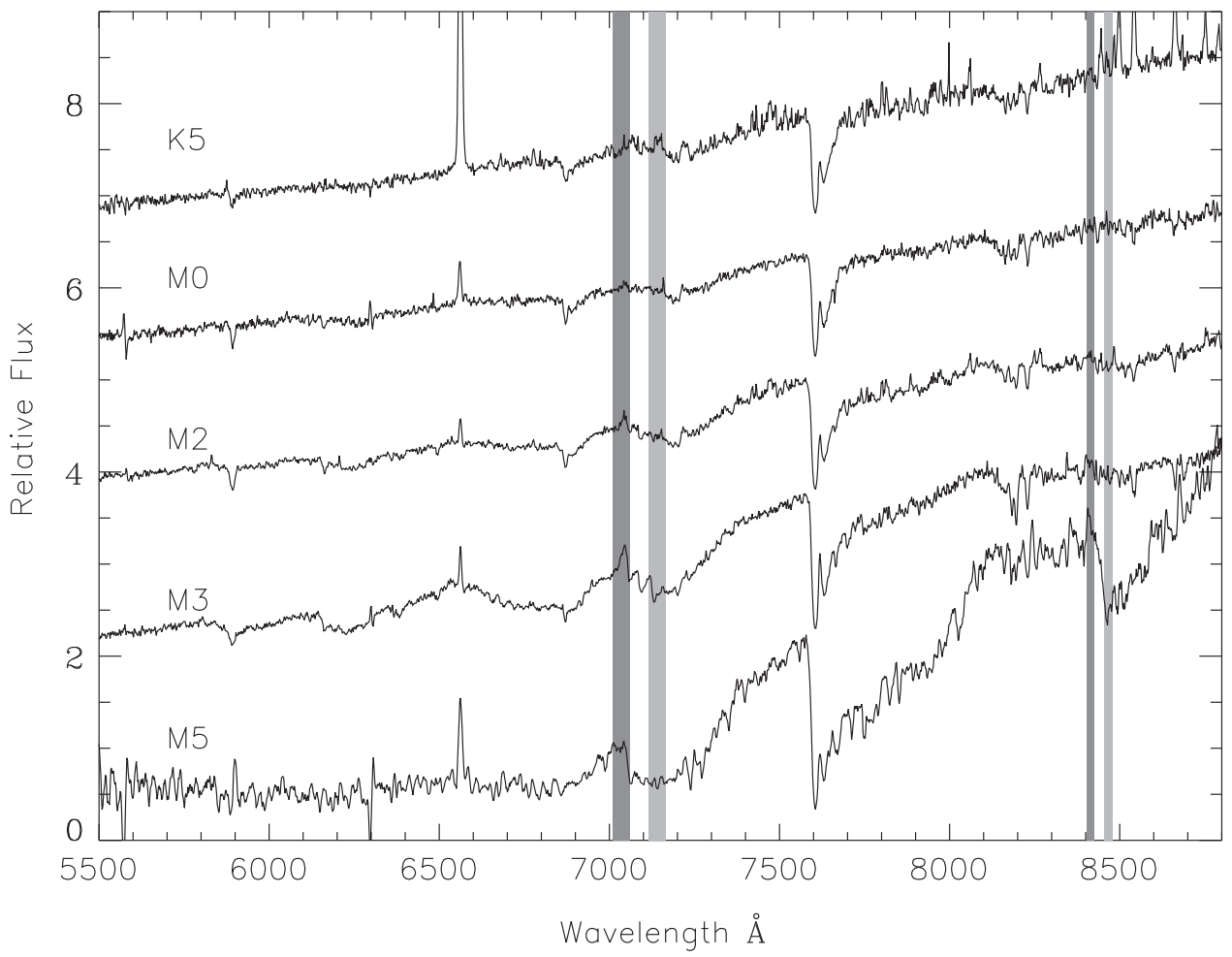

Figure 6. Examples of low-dispersion spectra of late-type cluster members obtained using HYDRA on WIYN. The sources are ordered by spectral type, classified using the temperature sensitive indices measuring the TiO bandhead absorption strengths at $\lambda 7140$ and $\lambda 8465$ (light gray) relative to nearby continuum levels at 7035 and $8415 \AA$ (dark gray).

the central $1024 \times 1024$ pixels of a Tektronix $2048 \times 2048$ pixel CCD, yielding a nominal field of view $\sim 5.5 \times 5.5$. The WFGS survey covered a region of about $150 \mathrm{arcmin}^{2}$, roughly centered upon $\mathrm{LkH} \alpha$ 234. Integration times for the slitless grism spectra were 120 and $1200 \mathrm{~s}$ for each field to ensure complete, unsaturated coverage for nearly all sources. The minimum measurable equivalent width of the WFGS spectra is about $2 \AA$.

\subsection{Low Dispersion Optical Spectroscopy}

Low-dispersion spectra were obtained using HYDRA on WIYN in 1993 and the RC Spectrograph on the Mayall $4 \mathrm{~m}$ telescope in 1994 by LAH. Spectra of earlier-type stars $(<\mathrm{K} 5)$ were classified using the standards of Allen \& Strom (1995). The relative strengths of a number of temperature sensitive features were adopted to include the $\mathrm{Na}$ I $\mathrm{D}$ doublet, the $\mathrm{Ca}$ I absorption lines between 6100 and $6200 \AA$, the blend near $\mathrm{H} \alpha$, and the $\mathrm{Ca}$ II near-infrared triplet, if in absorption. For late-type stars (K5 and later), the standards of Kirkpatrick et al. (1993) and the temperature sensitive TiO indices of Slesnick et al. (2006) and references therein were adopted. These indices measure the strength of $\mathrm{TiO}$ absorption features near $\lambda 7140$ and $\lambda 8465$ relative to narrow continuum passbands at 7035 and $8415 \AA$ respectively. The resulting indices were then compared with those of dwarf and pre-main sequence objects compiled by Slesnick et al. (2006). Shown in Figure 6 are examples of the WIYN spectra, ordered by spectral type with the regions used by the $\mathrm{TiO}$ indices for classification purposes demarcated in gray. The spectra were independently classified by both authors with LAH following methods similar to those described in Hillenbrand (1997). Where type or specific subclass could not be determined, ranges were estimated and are provided in Tables 1 and 3 .

\subsection{HIRES Observations}

High-dispersion optical spectra were obtained by G. H. Herbig for $\mathrm{BD}+65^{\circ} 1638, \mathrm{BD}+65^{\circ} 1637$, SVS 13 , and V350 Cep using the HIRES; (Vogt et al. 1994) on the Keck I telescope during the nights of 2003 July 06, 2004 July 24, 2010 June 06 , and 2007 July 22, respectively. The HIRES spectra of LkH $\alpha 234$ were obtained by LAH on 1999 December 06 and by J. Kuhn on 2004 June 13. HIRES was configured with the red cross-disperser and collimator in beam for all observations presented here. The $\mathrm{C} 1$ decker $\left(0,87 \times 7^{\prime \prime} \cdot 0\right)$, which has a projected slit width of 3 pixels, was used for the bulk of the spectra, providing a spectral resolution of $\sim 45,000$ $\left(\sim 6.7 \mathrm{~km} \mathrm{~s}^{-1}\right)$. Near-complete spectral coverage from $\sim 4340$ to $6800 \AA$ was achieved for the 2003 and 2004 observations, which were made with the single Tektronix $2048 \times 2048$ pixel CCD camera with $24 \mu \mathrm{m}$ pixels. The 1999 spectrum of $\mathrm{LkH} \alpha$ 234 used the D2 decker $\left(1^{\prime \prime} 148 \times 28^{\prime \prime} .0\right)$ and covers a range from 6240 to $8680 \AA$. The 2007 observations of V350 Cep were made with the upgraded three-chip mosaic of MIT-LL CCDs having $15 \mu \mathrm{m}$ pixels. The resulting spectral coverage of the 2007 observations of V350 Cep range from approximately 4300-8600 ̊. The CCDs were used in low-gain mode, resulting in readout noise levels of $\sim 2.8,3.1$, and $3.1 e^{-1}$ for the red, green, and blue detectors, respectively. Internal quartz lamps were used for flat fielding and ThAr lamp spectra were obtained for wavelength calibration. Integration times were $120-1200 \mathrm{~s}$, yielding signal-to-noise levels of up to $\sim 100$ for the early-type stars. The cross-dispersed spectra were reduced and extracted using the Mauna Kea Echelle Extraction pipeline 
Table 1

Optical and Infrared Photometry for $\mathrm{H} \alpha$ and X-ray Emission Sources in NGC 7129

\begin{tabular}{|c|c|c|c|c|c|c|c|c|c|c|c|c|c|c|c|c|c|c|c|c|c|}
\hline Identifier $^{a}$ & $\begin{array}{c}\alpha \\
(\mathrm{J} 2000)\end{array}$ & $\begin{array}{c}\delta \\
(\mathrm{J} 2000)\end{array}$ & $\mathrm{SpT}$ & $V^{b}$ & $V-R_{C}^{\mathrm{b}}$ & $V-I_{C}^{\mathrm{b}}$ & $J-H^{\mathrm{c}}$ & $H-K_{S}^{\mathrm{c}}$ & $K_{S}^{\mathrm{c}}$ & {$[3.6]^{\mathrm{d}}$} & {$[4.5]^{\mathrm{d}}$} & {$[5.8]^{\mathrm{d}}$} & {$[8.0]^{\mathrm{d}}$} & $w 1^{\mathrm{e}}$ & $w 2^{\mathrm{e}}$ & $w 3^{\mathrm{e}}$ & $w 4^{\mathrm{e}}$ & $\begin{array}{c}W \\
(\mathrm{H} \alpha)^{\mathrm{f}} \\
(\AA)\end{array}$ & $\begin{array}{c}W \\
(8542)^{\mathrm{g}} \\
(\AA)\end{array}$ & Other Identifiers & Notes $^{\mathrm{h}}$ \\
\hline MMN 1 & 214223.08 & +660604.4 & M3V & 20.47 & 1.56 & 3.11 & 1.01 & 0.66 & 13.39 & 12.51 & 12.14 & 11.78 & 11.02 & 12.39 & 12.02 & $\cdots$ & $\ldots$ & 32.9 & 0.3 & S3-U840 & $\ldots$ \\
\hline $\mathrm{IH} \alpha 763$ & 214226.60 & +6606 17.0 & K5 & 19.60 & 1.29 & 2.31 & 0.76 & 0.34 & 14.59 & $\ldots$ & & & & 14.00 & 14.08 & & $\ldots$ & 8.3 & & $\ldots$ & \\
\hline $\mathrm{IH} \alpha 764$ & 214229.57 & +660520.9 & G8-K2 & 19.72 & 0.96 & 1.80 & 0.50 & 0.82 & 15.25 & & & $\ldots$ & & & & $\ldots$ & $\ldots$ & 7.5 & & & \\
\hline $\mathrm{IH} \alpha 765$ & 214232.40 & +660459.1 & G5-K0 & 18.57 & 0.86 & 1.63 & 0.56 & 0.08 & 15.01 & $\cdots$ & $\cdots$ & $\cdots$ & $\cdots$ & 14.52 & 15.12 & $\ldots$ & $\ldots$ & 11.5 & ecr & $\cdots$ & $\ldots$ \\
\hline $\mathrm{IH} \alpha 766$ & 214234.72 & +660518.6 & $\mathrm{M} 3 \mathrm{~V}$ & 17.80 & 1.17 & 2.54 & 0.64 & 0.15 & 13.13 & 12.87 & 12.80 & 12.82 & 13.06 & 13.22 & 13.15 & $\ldots$ & $\ldots$ & 4.3 & ecr & S3-X4 & $\ldots$ \\
\hline MMN 2 & 214238.80 & +660635.8 & $\cdots$ & 20.68 & 2.22 & 3.60 & 1.32 & 0.97 & 12.51 & 11.01 & 10.59 & 10.42 & 10.19 & 10.96 & 10.49 & $\ldots$ & $\ldots$ & 437 & $\ldots$ & S3-U939, HH 242 & wk cont \\
\hline S3-X41 & 214238.91 & +660708.7 & $\ldots$ & 18.37 & 1.67 & 3.20 & 1.09 & 0.44 & 11.08 & & & $\ldots$ & & 10.42 & 10.33 & & & & & & \\
\hline $\mathrm{IH} \alpha 767$ & 214240.23 & +661328.7 & $\ldots$ & $\ldots$ & $\cdots$ & $\ldots$ & 0.94 & 0.58 & 12.51 & $\cdots$ & $\cdots$ & $\cdots$ & $\ldots$ & 11.74 & 11.05 & 8.92 & 6.70 & 206 & $\ldots$ & $\cdots$ & wk cont \\
\hline S3-X52 & 214240.34 & +66 10 07.2 & $\mathrm{A} 1$ & 12.44 & 0.33 & 0.73 & 0.30 & 0.13 & 10.58 & 10.52 & 10.52 & 10.27 & 9.73 & 10.43 & 10.37 & & & & & SVS 2 & $\ldots$ \\
\hline $\mathrm{IH} \alpha 768$ & 214240.49 & +660951.6 & $\ldots$ & 19.95 & 0.85 & 1.58 & & $\ldots$ & 14.87 & 13.12 & 12.33 & 11.54 & 10.48 & 13.13 & 11.92 & $\ldots$ & $\ldots$ & 86.0 & $\ldots$ & S3-U1612 & $\cdots$ \\
\hline MMN 3 & 214241.92 & +660924.5 & $\ldots$ & 21.19 & 1.82 & 3.78 & 0.84 & 0.35 & 13.99 & 13.56 & 13.29 & 13.08 & 12.30 & 13.66 & 13.23 & & & $22:$ & & S3-U1522 & wk cont \\
\hline $\mathrm{IH} \alpha 769$ & 214244.22 & +66 1007.0 & M4.5 V & 21.28 & 1.82 & 3.82 & 0.83 & 0.24 & 14.30 & $\ldots$ & $\ldots$ & $\ldots$ & $\ldots$ & 13.80 & 13.29 & $\ldots$ & $\ldots$ & 8.4 & 0.8 & $\ldots$ & $\ldots$ \\
\hline S3-X40 & 214245.32 & +660704.4 & & 20.91 & 1.63 & 3.42 & 1.02 & 0.59 & 13.39 & 13.13 & 13.05 & 12.35 & $\ldots$ & 11.39 & 10.90 & & & $\ldots$ & $\ldots$ & & $\ldots$ \\
\hline $\mathrm{IH} \alpha 770$ & 214245.51 & +660630.6 & G0: & 19.65 & 1.18 & 2.26 & 0.83 & 0.31 & 14.41 & $\ldots$ & & & $\ldots$ & & & $\ldots$ & $\ldots$ & 5.0 & abs & $\ldots$ & $\ldots$ \\
\hline $\mathrm{IH} \alpha 771$ & 214246.08 & +660556.2 & $\mathrm{M} 2 \mathrm{~V}$ & 18.17 & 1.31 & 2.60 & 0.77 & 0.27 & 12.75 & 12.58 & 12.60 & 12.57 & 13.15 & 12.56 & 12.38 & $\ldots$ & $\ldots$ & 5.3 & abs & S3-X31 & $\ldots$ \\
\hline $\mathrm{BD}+65^{\circ} 1636$ & 214246.09 & +660513.8 & $\mathrm{B} 8 \mathrm{~V}$ & 10.86 & 0.21 & 0.39 & 0.16 & 0.07 & 9.83 & 9.85 & 9.84 & 9.87 & 9.91 & 9.66 & 9.62 & $\ldots$ & $\ldots$ & $\ldots$ & $\ldots$ & S3-X3 & $\ldots$ \\
\hline $\mathrm{IH} \alpha 772$ & 214246.12 & +661345.8 & $\ldots$ & & $\ldots$ & $\ldots$ & 0.72 & 0.41 & 13.55 & $\ldots$ & & & $\ldots$ & 13.29 & 12.92 & 10.88 & & 50.1 & $\ldots$ & $\ldots$ & $\ldots$ \\
\hline $\mathrm{IH} \alpha 773$ & 214246.17 & +660656.6 & M0V & 19.65 & 1.45 & 2.86 & 0.77 & 0.27 & 12.75 & $\ldots$ & $\ldots$ & $\ldots$ & $\ldots$ & 12.56 & 12.38 & $\ldots$ & $\ldots$ & 9.5 & 0.2 & $\ldots$ & $\ldots$ \\
\hline $\mathrm{IH} \alpha 774$ & 214246.55 & +660633.4 & $\mathrm{K} 3$ & 19.42 & 1.24 & 2.16 & 0.70 & 0.56 & 14.35 & & $\ldots$ & $\ldots$ & & $\cdots$ & $\ldots$ & $\ldots$ & $\ldots$ & 4.2 & abs & & $\ldots$ \\
\hline $\mathrm{IH} \alpha 775$ & 214246.59 & +660622.3 & M5V & 21.63 & 1.30 & 3.33 & 0.74 & 0.40 & 14.98 & $\ldots$ & $\ldots$ & $\ldots$ & $\ldots$ & $\ldots$ & $\ldots$ & $\ldots$ & $\ldots$ & 8: & flat & $\ldots$ & $\ldots$ \\
\hline $\mathrm{IH} \alpha 776$ & 214246.87 & +660657.4 & м0 & 19.65 & 1.45 & 2.86 & 1.02 & 0.51 & 12.09 & 11.24 & 10.91 & 10.55 & 9.74 & & $\ldots$ & & & 25.9 & abs & S3-X39 & $\ldots$ \\
\hline $\mathrm{IH} \alpha 777$ & 214247.05 & +660457.8 & $\ldots$ & 15.54 & 1.02 & 2.00 & 0.77 & 0.28 & 11.05 & 10.79 & 10.74 & 10.54 & 10.00 & 10.67 & 10.37 & $\ldots$ & $\ldots$ & 2.5 & $\ldots$ & S3-X2 & $\ldots$ \\
\hline $\mathrm{IH} \alpha 778$ & 214247.46 & +660703.4 & $\mathrm{M} 2 \mathrm{~V}$ & 21.66 & 1.72 & 3.84 & 1.02 & 0.52 & 13.47 & & $\cdots$ & $\cdots$ & & $\cdots$ & $\cdots$ & $\cdots$ & $\ldots$ & 20.8 & 0.7 & & $\ldots$ \\
\hline $\mathrm{IH} \alpha 779$ & 214247.90 & +66 0653.0 & $\ldots$ & 17.91 & 1.28 & 2.53 & 1.04 & 0.33 & 12.09 & 11.69 & 11.71 & 10.89 & $\ldots$ & $\ldots$ & $\ldots$ & $\ldots$ & $\ldots$ & 4.4 & $\ldots$ & S3-X23 & $\ldots$ \\
\hline $\mathrm{IH} \alpha 780$ & 214248.13 & +66 0743.2 & K5: & 21.42 & 1.76 & 3.22 & 1.01 & 0.37 & 14.31 & $\ldots$ & & $\ldots$ & $\ldots$ & $\ldots$ & $\ldots$ & $\ldots$ & $\ldots$ & 4.9 & abs & $\ldots$ & $\ldots$ \\
\hline $\mathrm{IH} \alpha 781$ & 214249.87 & +66 0542.6 & $\ldots$ & 20.02 & 1.36 & 3.29 & 0.64 & 0.29 & 13.72 & 13.53 & 13.39 & $\ldots$ & $\ldots$ & $\ldots$ & $\ldots$ & $\ldots$ & $\ldots$ & 4: & $\ldots$ & S3-X8 & $\mathrm{em}$ ? \\
\hline $\mathrm{IH} \alpha 782$ & 214249.93 & +66 0554.6 & M4 & 21.61 & 2.10 & 4.08 & 0.74 & 0.45 & 14.09 & & $\cdots$ & $\cdots$ & & $\cdots$ & $\ldots$ & $\ldots$ & $\ldots$ & 18.3 & abs & & $\ldots$ \\
\hline $\mathrm{BD}+65^{\circ} 1637$ & 214250.18 & +6606 35.1 & B3e & 10.53 & 0.24 & 0.51 & 0.24 & 0.26 & 8.47 & 8.13 & 7.90 & 7.57 & 7.09 & 7.90 & 7.51 & 6.17 & 0.34 & 19.6 & $\ldots$ & S3-X13 & $\ldots$ \\
\hline $\mathrm{IH} \alpha 783$ & 214250.92 & +660603.6 & $\mathrm{M} 2 \mathrm{~V}$ & 17.73 & 1.21 & 2.38 & 0.85 & 0.24 & 12.55 & 12.42 & 12.37 & 12.24 & $\ldots$ & $\ldots$ & $\ldots$ & $\ldots$ & $\ldots$ & 5.3 & abs & S3-X11 & $\ldots$ \\
\hline $\mathrm{IH} \alpha 784$ & 214250.99 & +660359.2 & $\ldots$ & 19.96 & 1.01 & 1.88 & $\ldots$ & $\ldots$ & $\ldots$ & $\ldots$ & $\ldots$ & $\ldots$ & $\ldots$ & 15.22 & 14.07 & $\ldots$ & $\ldots$ & 8: & $\ldots$ & $\ldots$ & wk cont \\
\hline MMN 5 & 214251.42 & +66 0556.2 & $\ldots$ & 21.82 & 1.56 & 3.03 & 1.07 & 0.56 & 13.57 & 12.62 & 12.09 & 11.52 & 10.53 & $\ldots$ & $\cdots$ & $\cdots$ & $\ldots$ & 280 & $\ldots$ & S3-U815 & wk cont \\
\hline S3-X35 & 214251.96 & +6606 33.4 & & 18.06 & 0.99 & 2.39 & 0.73 & 0.18 & 13.13 & 12.63 & 12.68 & $\ldots$ & $\ldots$ & & $\ldots$ & & & $\ldots$ & $\ldots$ & $\ldots$ & $\ldots$ \\
\hline $\mathrm{IH} \alpha 785$ & 214252.30 & +660535.3 & M1V & 18.98 & 1.30 & 2.70 & 0.86 & 0.32 & 13.19 & 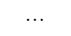 & $\ldots$ & $\ldots$ & $\ldots$ & $\ldots$ & $\ldots$ & $\ldots$ & $\ldots$ & 3.7 & abs & $\ldots$ & $\ldots$ \\
\hline MMN 6 & 214252.61 & +660657.2 & M1V & 18.95 & 1.48 & 2.94 & 1.17 & 0.85 & 11.80 & 10.81 & 10.24 & 9.77 & 9.15 & & & $\ldots$ & $\ldots$ & 69.2 & 2.5 & S3-X25 & \\
\hline MMN 7 & 214253.14 & +660714.8 & $\ldots$ & 20.58 & 1.12 & 1.94 & 1.42 & 1.23 & 12.93 & 11.61 & 10.93 & 10.41 & 9.57 & $\ldots$ & $\ldots$ & $\ldots$ & $\ldots$ & 201 & 24.6 & S3-U1085 & wk cont \\
\hline $\mathrm{IH} \alpha 786$ & 214253.21 & +660720.8 & & 19.30 & 1.42 & 2.71 & 1.15 & 0.51 & 12.68 & 11.62 & 11.29 & 10.82 & 9.92 & & $\ldots$ & & & 4.2 & $\ldots$ & S3-U1109 & $\ldots$ \\
\hline MMN 8 & 214253.45 & +660919.6 & $\ldots$ & 21.87 & 1.71 & 2.77 & 1.49 & 0.92 & 14.58 & 12.91 & 12.36 & 11.87 & 11.23 & 13.25 & 12.47 & $\ldots$ & $\ldots$ & 234 & $\ldots$ & S3-U1504 & wk cont \\
\hline MMN 9 & 214253.49 & +660805.3 & $\mathrm{M} 2 \mathrm{~V}$ & 17.55 & 1.26 & 2.50 & 0.85 & 0.24 & 12.12 & 11.85 & 11.78 & 11.22 & & 11.22 & 11.16 & $\ldots$ & & 2.6 & abs & S3-X29 & \\
\hline S3-X45 & 214254.08 & +660814.9 & G8 & 19.80 & 1.57 & 2.98 & 1.17 & 0.43 & 12.82 & 12.52 & 12.55 & 12.55 & 12.29 & $\ldots$ & $\ldots$ & $\ldots$ & $\ldots$ & $\ldots$ & $\ldots$ & $\ldots$ & $\ldots$ \\
\hline $\mathrm{IH} \alpha 787$ & 214254.71 & +660635.6 & M0V & 19.00 & 1.43 & 2.59 & 1.00 & 0.62 & 12.56 & 11.66 & 11.37 & 11.02 & 10.05 & $\ldots$ & $\ldots$ & $\ldots$ & $\ldots$ & 5.4 & abs & S3-X36 & $\ldots$ \\
\hline MMN 10 & 214254.80 & +660612.7 & $\ldots$ & 19.01 & 1.38 & 2.59 & 0.94 & 0.35 & 12.91 & 12.58 & $\ldots$ & $\ldots$ & $\ldots$ & $\ldots$ & $\ldots$ & $\ldots$ & $\ldots$ & $\mathrm{em} ?$ & $\ldots$ & S3-X32 & wk cont \\
\hline $\mathrm{IH} \alpha 788$ & 214254.87 & +660631.4 & M1V & 19.42 & 1.53 & 2.90 & 1.05 & 0.27 & 13.42 & & & & & & & & & 4.9 & abs & & \\
\hline $\mathrm{IH} \alpha 789$ & 214254.89 & +660721.3 & M3V & 20.00 & 1.71 & 3.40 & 1.05 & 0.35 & 12.84 & 12.50 & 12.44 & 11.96 & $\ldots$ & & & $\ldots$ & & 5.8 & abs & S3-X26 & \\
\hline S3-X37 & 214255.72 & +660645.0 & $\ldots$ & 21.16 & 1.61 & 3.64 & 1.00 & 0.31 & 14.27 & 13.62 & 13.37 & $\ldots$ & $\ldots$ & $\ldots$ & $\ldots$ & $\ldots$ & $\ldots$ & $\ldots$ & $\ldots$ & $\ldots$ & $\ldots$ \\
\hline $\mathrm{IH} \alpha 790$ & 214255.76 & +66 0542.9 & M3. $5 \mathrm{~V}$ & 20.41 & 0.74 & 2.81 & 0.85 & 0.40 & 13.97 & 13.43 & 13.38 & $\ldots$ & $\ldots$ & $\cdots$ & $\ldots$ & $\cdots$ & $\ldots$ & 5.7 & ecr & S3-X48 & $\ldots$ \\
\hline $\mathrm{IH} \alpha 791$ & 214255.86 & +660721.1 & M1V & 20.70 & 1.69 & 3.28 & 1.31 & 0.82 & 12.47 & 11.16 & 10.54 & 9.98 & 9.02 & $\ldots$ & $\ldots$ & $\ldots$ & $\ldots$ & 28.1 & 8.6 & S3-U1107 & $\ldots$ \\
\hline MMN 11 & 214256.25 & +660602.1 & $\ldots$ & 15.75 & 0.95 & 1.85 & 0.77 & 0.25 & 11.41 & 11.28 & 11.13 & 10.78 & $\ldots$ & & & & & $\mathrm{em} ?$ & & S3-X10 & \\
\hline S3-X18 & 214256.77 & +660637.2 & $\ldots$ & 17.93 & 1.31 & 2.57 & 0.98 & 0.48 & 12.21 & 11.47 & 11.21 & 10.44 & $\ldots$ & $\ldots$ & $\ldots$ & $\ldots$ & $\ldots$ & $\ldots$ & $\ldots$ & $\ldots$ & $\ldots$ \\
\hline IH $\alpha 792$ & 214257.15 & +660634.9 & K5 & 17.18 & 1.11 & 2.11 & 0.92 & 0.22 & 12.53 & $\cdots$ & 12.00 & & $\ldots$ & & & & & 1.6 & abs & S3-X15 & \\
\hline $\mathrm{IH} \alpha 793$ & 214257.40 & +6607 15.1 & M3V & 19.72 & 1.69 & 3.38 & 0.81 & 0.36 & 12.98 & $\ldots$ & $\ldots$ & $\ldots$ & $\ldots$ & $\ldots$ & $\ldots$ & $\ldots$ & $\ldots$ & 3.2 & abs & $\ldots$ & $\ldots$ \\
\hline MMN 12 & 214258.10 & +660739.3 & M5V & 20.49 & 1.94 & 3.93 & 0.77 & 0.50 & 13.03 & & & & & & & $\ldots$ & & 13.6 & flat & & \\
\hline $\mathrm{IH} \alpha 794$ & 214258.18 & +660540.5 & $\mathrm{M} 2 \mathrm{~V}$ & 18.64 & 1.09 & 2.27 & 0.80 & 0.13 & 13.20 & $\cdots$ & $\ldots$ & $\cdots$ & $\ldots$ & $\ldots$ & $\ldots$ & $\ldots$ & $\ldots$ & 8.9 & 4.8 & S3-X47 & $\ldots$ \\
\hline S3-X43 & 214258.34 & +660726.3 & & 22.68 & 2.53 & 4.93 & 1.69 & 0.71 & 11.91 & 11.44 & 11.39 & 10.93 & & & & & & & & & $\ldots$ \\
\hline $\mathrm{IH} \alpha 795$ & 214258.36 & +660527.3 & M2.5 V & 18.88 & 1.30 & 2.75 & 0.93 & 0.39 & 12.64 & 11.79 & 11.39 & $\ldots$ & $\ldots$ & $\ldots$ & $\ldots$ & $\ldots$ & $\ldots$ & 18.6 & 0.5 & S3-X6 & $\ldots$ \\
\hline $\mathrm{BD}+65^{\circ} 1638$ & 214258.60 & +660610.3 & B3V & 10.42 & 0.33 & 0.69 & 0.32 & 0.15 & 8.51 & 8.50 & 8.48 & 8.42 & 8.00 & & & & & & & S3-X13, SVS 8 & \\
\hline S3-X17 & 214258.77 & +660636.8 & $\ldots$ & 17.84 & 1.19 & 2.46 & 0.93 & 0.22 & 12.34 & 11.98 & 11.85 & $\ldots$ & $\ldots$ & $\ldots$ & $\ldots$ & $\ldots$ & $\ldots$ & $\ldots$ & $\ldots$ & $\ldots$ & $\ldots$ \\
\hline $\mathrm{IH} \alpha 796$ & 214259.41 & +66 1112.3 & & $\ldots$ & & $\ldots$ & 0.76 & 0.53 & 14.77 & $\cdots$ & $\ldots$ & $\cdots$ & $\cdots$ & & $\cdots$ & & & 50: & & & wk cont \\
\hline HBC 731 & 214259.61 & +660433.8 & M1.5 V & 18.69 & 1.58 & 3.08 & 1.26 & 0.68 & 10.95 & 10.18 & 9.73 & 9.39 & 8.91 & 10.20 & 9.59 & 7.58 & 5.60 & 54.9 & 4.5 & S3-X1 & \\
\hline $\mathrm{IH} \alpha 798$ & 214259.97 & +66 0101.0 & $\ldots$ & $\ldots$ & $\ldots$ & $\ldots$ & 0.84 & 0.20 & 13.67 & $\ldots$ & $\ldots$ & $\ldots$ & $\ldots$ & 13.60 & 13.47 & $\ldots$ & $\ldots$ & $\mathrm{em}$ ? & $\ldots$ & $\ldots$ & $\ldots$ \\
\hline
\end{tabular}


Table 1

(Continued)

\begin{tabular}{|c|c|c|c|c|c|c|c|c|c|c|c|c|c|c|c|c|c|c|c|c|c|}
\hline Identifier $^{a}$ & $\begin{array}{c}\alpha \\
(\mathrm{J} 2000)\end{array}$ & $\begin{array}{c}\delta \\
(\mathrm{J} 2000)\end{array}$ & SpT & $V^{b}$ & $V-R_{C}^{\mathrm{b}}$ & $V-I_{C}^{\mathrm{b}}$ & $J-H^{\mathrm{c}}$ & $H-K_{S}^{\mathrm{c}}$ & $K_{S}^{\mathrm{c}}$ & {$[3.6]^{\mathrm{d}}$} & {$[4.5]^{\mathrm{d}}$} & {$[5.8]^{\mathrm{d}}$} & {$[8.0]^{\mathrm{d}}$} & $w 1^{\mathrm{e}}$ & $w 2^{\mathrm{e}}$ & $w 3^{\mathrm{e}}$ & $w 4^{\mathrm{e}}$ & $\begin{array}{c}W \\
(\mathrm{H} \alpha)^{\mathrm{f}} \\
(\AA)\end{array}$ & $\begin{array}{c}W \\
(8542)^{\mathrm{g}} \\
(\AA)\end{array}$ & Other Identifiers & Notes $^{\mathrm{h}}$ \\
\hline $\mathrm{IH} \alpha 799$ & 214259.98 & +660642.4 & M3V & 19.69 & 1.47 & 3.26 & 0.86 & 0.34 & 13.15 & 12.73 & 13.05 & $\ldots$ & $\ldots$ & $\ldots$ & $\cdots$ & $\ldots$ & $\ldots$ & 7.0 & ecr & S3-X19 & $\ldots$ \\
\hline HBC 732 & 214300.00 & +661127.9 & M2 & 16.35 & 1.01 & 2.11 & 1.02 & 0.68 & 11.01 & $\ldots$ & $\ldots$ & $\ldots$ & $\ldots$ & 10.06 & 9.13 & 6.58 & 4.10 & 27.7 & $\ldots$ & MMN 13, V350 Cep & $\ldots$ \\
\hline MMN 14 & 214300.23 & +66 0647.4 & M0V & 20.02 & 1.53 & 2.73 & 1.38 & 0.69 & 12.09 & & $\ldots$ & & $\ldots$ & 9.53 & 9.33 & & & 92.7 & 9.3 & $\ldots$ & \\
\hline SVS 13 & 214301.71 & +660708.9 & B5 & 14.57 & 0.86 & 1.78 & 0.68 & 0.31 & 10.25 & 9.19 & 8.81 & $\ldots$ & $\ldots$ & 7.64 & 7.14 & $\ldots$ & $\ldots$ & $\mathrm{em}$ & ecr & S3-X51 & $\ldots$ \\
\hline $\mathrm{IH} \alpha 800$ & 214301.88 & +6606 44.7 & $\mathrm{~K} 3-5 \mathrm{~V}$ & 17.24 & 1.18 & 2.22 & 0.89 & 0.40 & 12.02 & 11.12 & 10.80 & 11.15 & & & & & $\ldots$ & 3.8 & abs & $\mathrm{S} 3-\mathrm{X} 20$ & $\ldots$ \\
\hline MMN 15 & 214302.46 & +660703.9 & $\ldots$ & 19.64 & 1.34 & 2.55 & 0.90 & 0.85 & 12.26 & $\ldots$ & $\ldots$ & $\ldots$ & $\ldots$ & $\ldots$ & $\ldots$ & $\ldots$ & $\ldots$ & 9: & $\ldots$ & $\ldots$ & $\ldots$ \\
\hline S3-X50 & 214303.01 & +660655.9 & & $\ldots$ & $\ldots$ & & & & & 12.79 & 12.59 & & $\ldots$ & & $\ldots$ & & & & & $\ldots$ & \\
\hline $\mathrm{IH} \alpha 801$ & 214303.20 & +66 11 15.0 & $\ldots$ & $\ldots$ & $\ldots$ & $\ldots$ & 1.16 & 1.12 & 14.34 & $\ldots$ & $\ldots$ & $\ldots$ & $\ldots$ & 11.70 & 9.90 & 6.01 & 3.33 & 111 & $\ldots$ & GGD $33 \mathrm{~A}$ & $\ldots$ \\
\hline $\mathrm{IH} \alpha 802$ & 214303.43 & +66 0526.4 & M2 & 17.86 & 1.24 & 2.49 & 0.98 & 0.53 & 12.01 & $\ldots$ & $\ldots$ & $\ldots$ & & $\ldots$ & $\ldots$ & $\ldots$ & $\ldots$ & 59.3 & 4.5 & & $\ldots$ \\
\hline $\mathrm{IH} \alpha 803$ & 214304.71 & +660030.5 & & & $\ldots$ & $\ldots$ & 1.16 & 0.62 & 13.51 & & $\ldots$ & & $\ldots$ & 12.93 & 12.31 & 10.68 & & 28: & & $\ldots$ & wk con \\
\hline $\mathrm{IH} \alpha 804$ & 214304.95 & +66 0653.6 & $\mathrm{~K} 2 \mathrm{~V}$ & 17.90 & 1.42 & 2.58 & 1.08 & 0.72 & 11.26 & $\ldots$ & $\ldots$ & $\ldots$ & $\ldots$ & $\ldots$ & & $\ldots$ & $\ldots$ & 6.3 & abs & S3-X22 & $\ldots$ \\
\hline $\mathrm{IH} \alpha 805$ & 214305.09 & +660929.5 & M7 & 22.45 & 1.93 & 4.05 & 0.64 & 0.26 & 14.90 & 14.23 & 14.07 & 13.70 & 13.11 & 14.62 & 14.33 & $\ldots$ & $\ldots$ & 14.9 & abs & S3-U1542 & $\ldots$ \\
\hline LkH $\alpha 234$ & 214306.82 & +66 0654.2 & B8 & 12.48 & 0.66 & 1.34 & 1.33 & 1.12 & 7.08 & 5.79 & 5.15 & 4.45 & 3.33 & 4.69 & 2.90 & 1.32 & -1.75 & 56.6 & 9.3 & MMN 16 & $\ldots$ \\
\hline $\mathrm{IH} \alpha 806$ & 214311.41 & +66 1255.5 & & $\ldots$ & $\ldots$ & $\ldots$ & 1.02 & 0.60 & 12.70 & & $\ldots$ & & $\ldots$ & 11.89 & 11.26 & 9.43 & 7.32 & 166 & & $\ldots$ & \\
\hline MMN 17 & 214311.61 & +66 0911.4 & M1V & 16.30 & 1.08 & 2.16 & 0.80 & 0.31 & 11.49 & 11.04 & 10.73 & 10.38 & 9.70 & 11.16 & 10.79 & 8.82 & 7.03 & 4.8 & abs & S3-X30 & $\ldots$ \\
\hline IH $\alpha 807$ & 214312.26 & +660605.8 & M5V & 21.65 & 1.48 & 3.80 & 0.74 & 0.40 & 13.95 & 13.36 & 13.05 & 12.48 & & & & $\ldots$ & $\ldots$ & 7.0 & flat & S3-U849 & \\
\hline $\mathrm{IH} \alpha 808$ & $2143 \quad 12.35$ & +660955.4 & M4.5 & 19.71 & 1.52 & 3.08 & 0.91 & 0.31 & 13.59 & 13.00 & 12.74 & 12.39 & 11.85 & 13.15 & 12.73 & 11.18 & $\ldots$ & 17.4 & ecr & S2-U1640 & $\ldots$ \\
\hline $\mathrm{IH} \alpha 809$ & 214314.36 & +66 0859.2 & M4V & 22.48 & 1.62 & 4.21 & 0.73 & 0.37 & 14.93 & 14.23 & 13.89 & 13.59 & 12.98 & & & & & 36.9 & abs & S3-U1433 & $\ldots$ \\
\hline $\mathrm{IH} \alpha 810$ & 214314.43 & +66 0734.8 & M3 & 20.50 & 1.41 & 2.95 & 1.18 & 0.49 & 13.84 & 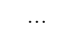 & & & & 12.89 & 10.98 & $\ldots$ & $\ldots$ & 2.9 & flat & & $\ldots$ \\
\hline $\mathrm{IH} \alpha 811$ & 214315.28 & +6607 57.1 & M2.5 V & 21.31 & 1.66 & 3.38 & 1.67 & 0.75 & 12.59 & 11.57 & 11.03 & 10.41 & 9.41 & $\ldots$ & $\ldots$ & $\ldots$ & $\ldots$ & 6.2 & flat & S3-X28 & $\ldots$ \\
\hline MMN 19 & 214316.83 & +660548.6 & M2V & 18.31 & 1.29 & 2.48 & 0.88 & 0.18 & 13.12 & 12.74 & 12.57 & 12.45 & 11.99 & $\ldots$ & $\ldots$ & $\ldots$ & $\ldots$ & 15.8 & abs & S3-X9 & $\ldots$ \\
\hline $\mathrm{IH} \alpha 812$ & 214318.06 & +66 0535.2 & M3V & 19.74 & 1.47 & 3.06 & 0.77 & 0.29 & 13.70 & 13.39 & 13.27 & 13.29 & 13.12 & $\ldots$ & $\ldots$ & $\ldots$ & $\ldots$ & 3.6 & flat & S3-X7 & $\ldots$ \\
\hline $\mathrm{IH} \alpha 813$ & 214319.37 & +660721.5 & M4.5 V & 20.70 & 1.67 & 3.50 & 0.77 & 0.40 & 13.89 & 13.90 & 13.72 & 13.62 & 13.43 & & & & $\ldots$ & 17.5 & 0.8 & S3-X42 & $\ldots$ \\
\hline $\mathrm{IH} \alpha 814$ & 214321.07 & +660622.8 & $\ldots$ & 17.83 & 1.23 & 2.56 & 0.66 & 0.15 & 12.75 & 12.44 & $\ldots$ & 12.32 & 12.42 & $\ldots$ & $\ldots$ & $\ldots$ & $\ldots$ & $2:$ & abs & S3-X14 & binary \\
\hline S2-X2 & 214324.89 & +66 0734.1 & $\ldots$ & 23.25 & 1.44 & 3.00 & & . & . & 17.12 & 16.15 & & & $\ldots$ & & $\ldots$ & $\ldots$ & $\ldots$ & $\ldots$ & & $\ldots$ \\
\hline $\mathrm{IH} \alpha 815$ & 214326.95 & +660936.5 & M0V & 16.49 & 0.87 & 1.67 & 0.71 & 0.18 & 12.72 & 12.67 & 12.66 & 12.60 & 12.55 & 12.73 & 12.74 & $\ldots$ & $\ldots$ & 1.6 & ecr & S2-X4 & $\ldots$ \\
\hline $\mathrm{IH} \alpha 816$ & 214328.10 & +66 0057.2 & & $\ldots$ & & $\ldots$ & 0.95 & 0.33 & 12.55 & $\ldots$ & $\ldots$ & $\ldots$ & $\ldots$ & 12.25 & 11.91 & 10.77 & & 15.0 & $\ldots$ & $\ldots$ & $\ldots$ \\
\hline S3-X56 & 214329.28 & +660331.5 & G6 & 11.17 & 0.31 & 0.58 & 0.29 & 0.04 & 9.69 & 9.69 & 9.69 & 9.64 & 9.64 & 9.64 & 9.65 & 10.13 & 9.41 & $\ldots$ & $\ldots$ & SVS 16 & $\ldots$ \\
\hline MMN 20 & 214331.82 & +660850.6 & & 18.21 & 1.20 & 2.34 & 1.04 & 0.60 & 12.30 & 11.59 & 11.29 & 11.02 & 10.31 & 11.88 & 11.47 & & & 21.1 & & $\mathrm{~S} 2-\mathrm{X} 1$ & \\
\hline $\mathrm{IH} \alpha 817$ & 214336.87 & +66 0752.6 & K5: & 19.33 & 1.16 & 2.19 & 0.70 & 0.45 & 14.40 & $\ldots$ & $\ldots$ & & $\ldots$ & 14.23 & 14.19 & $\ldots$ & $\ldots$ & 4.4 & abs & & $\ldots$ \\
\hline MMN 22 & 214343.44 & $\begin{array}{r}+660730.8 \\
\end{array}$ & $\ldots$ & 18.57 & 1.38 & 2.70 & 1.05 & 0.46 & 12.15 & 11.43 & 11.05 & 10.63 & 10.06 & 11.65 & 11.16 & 9.60 & & $\mathrm{em}$ ? & & S2-X5 & \\
\hline $\mathrm{IH} \alpha 818$ & 214343.71 & +660822.3 & M3 & 19.61 & 1.45 & 2.95 & 0.90 & 0.25 & 13.66 & $\ldots$ & $\ldots$ & $\ldots$ & $\ldots$ & $\ldots$ & $\ldots$ & $\ldots$ & $\ldots$ & 7.3 & flat & $\ldots$ & $\ldots$ \\
\hline $\mathrm{IH} \alpha 819$ & 214405.37 & +66 0553.1 & K5 & 18.70 & 1.09 & 2.15 & 1.54 & 0.93 & 11.22 & 9.83 & 9.33 & 8.92 & 8.16 & 10.09 & 9.38 & 7.32 & 4.94 & 60.6 & 5.3 & S2-U820 & \\
\hline $\mathrm{IH} \alpha 820$ & 214406.34 & +66 0423.1 & $\ldots$ & 18.71 & 1.36 & 2.62 & 1.13 & 0.69 & 11.38 & $\ldots$ & $\ldots$ & $\ldots$ & $\ldots$ & 10.51 & 10.00 & 7.96 & 5.68 & 2.7 & $\ldots$ & $\ldots$ & $\ldots$ \\
\hline $\mathrm{IH} \alpha 821$ & 214407.82 & +66 0433.2 & M3.5 & 19.57 & 1.59 & 3.16 & 0.94 & 0.31 & 13.21 & $\ldots$ & $\ldots$ & $\ldots$ & $\ldots$ & 12.70 & 12.26 & $\ldots$ & $\ldots$ & 25.4 & ecr & $\ldots$ & $\ldots$ \\
\hline
\end{tabular}

Notes.

IH $\alpha$ number unless previously identified by Magakian et al. (2004) or the Herbig Bell Catalog (HBC).

Optical photometry from the UH $2.2 \mathrm{~m}$ or the KPNO $0.9 \mathrm{~m}$.

${ }^{\mathrm{c}}$ Near-infrared photometry from the 2MASS Point Source Catalog.

${ }^{\mathrm{d}}$ Spitzer IRAC photometry from Stelzer \& Scholz (2009).

WISE photometry from the AllWISE Source Catalog.

${ }^{\mathrm{f}}$ Positive values indicate emission.

${ }^{g}$ Positive values indicate emission; abs-Ca II $\lambda 8542$ in absorption; ecr-emission core reversal is evident; flat-flat continuum noted at $\lambda 8542$.

h wk cont: weak continuum on WFGS images. 

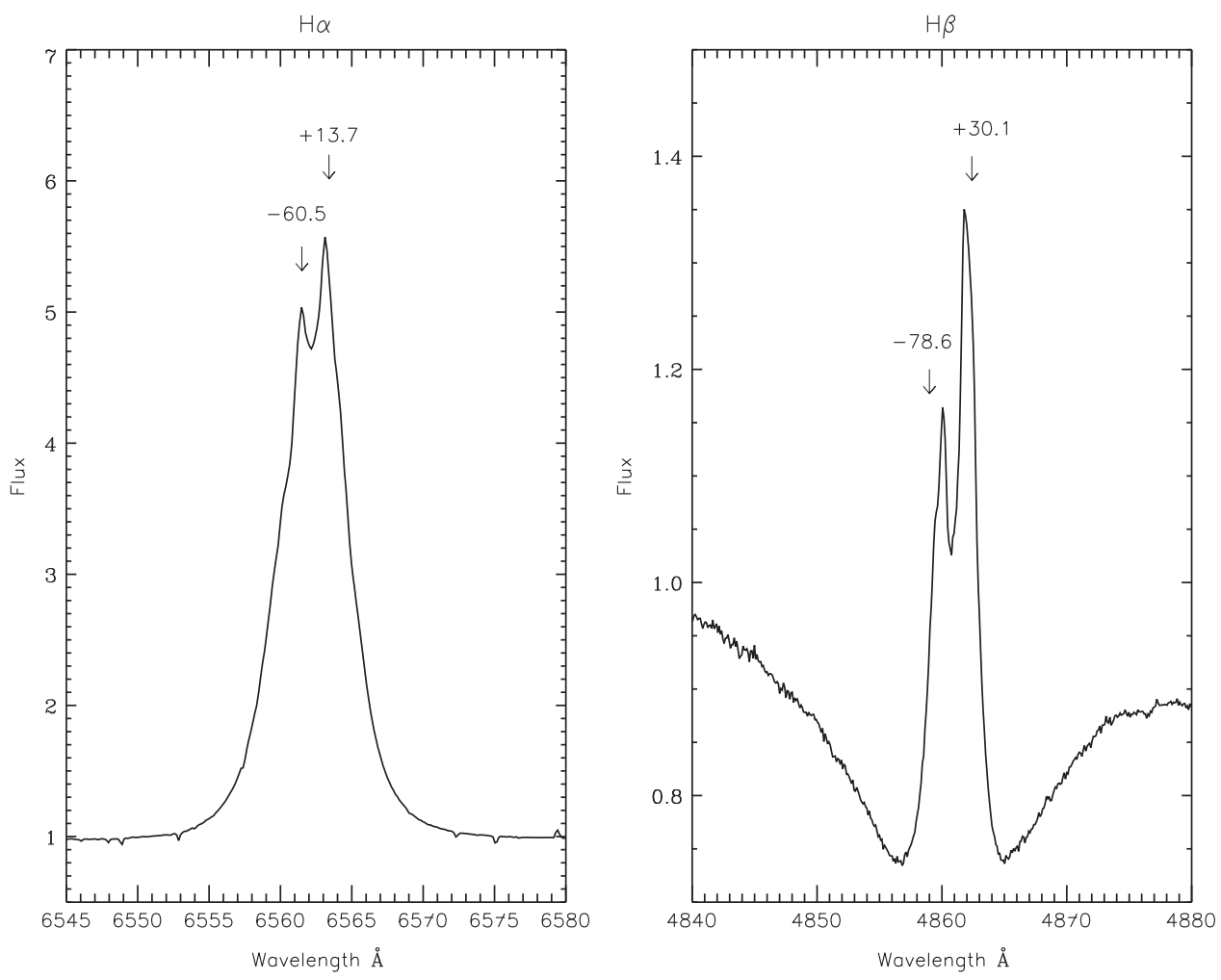

Figure 7. Sections of the HIRES spectrum of the $\mathrm{B} 3 \mathrm{e}$ star $\mathrm{BD}+65^{\circ} 1637$ centered near $\mathrm{H} \alpha$ (left) and $\mathrm{H} \beta$ (right). Strong $\mathrm{H} \alpha$ emission $(W=-25 \AA)$ is apparent with wings extending beyond $\pm 550 \mathrm{~km} \mathrm{~s}^{-1}$. There is no indication of an underlying photospheric absorption profile. The emission line is double-peaked, and the radial velocities of the red and blue peaks are annotated in the figure. $\mathrm{H} \beta$ reveals a similar double-peaked emission structure, but is substantially weaker $(W=-2.4 \AA)$ and reveals the wings of a broad photospheric absorption profile.

written by T. Barlow. Standard routines available through IRAF and IDL were used for spectral analysis. The raw as well as pipeline extracted spectra for the HIRES observations presented here are available through the Keck Observatory Archive: https://koa.ipac.caltech.edu.

\subsection{Adaptive Optics Imaging of LkHo 234}

High angular resolution, near-infrared imaging of $\mathrm{LkH} \alpha 234$ was obtained with NIRC2 using the natural guide star (NGS) adaptive optics system on the Keck II telescope on 2012 June 30 by SED. The images were obtained with the wide camera $\left(0^{\prime \prime} 04\right.$ pixel $^{-1}$ platescale $)$ in beam yielding a $40^{\prime \prime} \times 40^{\prime \prime}$ field of view. A three position dither was used to image the field, avoiding the quadrant of the Alladin III detector that exhibits a higher level of fixed pattern noise. Integration times were $10 \mathrm{~s}$ with 10 coadds in all three filters $\left(J, H, K^{\prime}\right)$, yielding an effective integration time of $100 \mathrm{~s}$ per frame. Basic image reduction and analysis were completed using standard routines written in IDL.

\section{ANALYSIS OF THE HIGH DISPERSION SPECTROSCOPY IN THE CONTEXT OF CIRCUMSTELLAR ENVIRONMENTS}

\section{1. $B D+65^{\circ} 1637$}

$\mathrm{BD}+65^{\circ} 1637$ was recognized by Merrill \& Burwell (1950) as a moderate intensity $\mathrm{H} \alpha$ emission star. Herbig (1960) found similar emission on plates obtained at Lick Observatory and classified the star as B5n or earlier. Narrow emission components were present near the centers of all Balmer lines from $\mathrm{H} \beta$ through $\mathrm{H} \delta$ and possibly extending to $\mathrm{H} \epsilon$. Herbig (1960) also noted that interstellar Ca II was present and that the stellar spectrum was suggestive of an ordinary Be star. Other classifications for $\mathrm{BD}+65^{\circ} 1637$ taken from the literature include: B2-B3 (Strom et al. 1972), B5 (Finkenzeller 1985), and B3 (Hillenbrand et al. 1992). Strom et al. (1972) found $\mathrm{H} \beta$ to be in emission with no obvious P Cygni component and noted that $\mathrm{BD}+65^{\circ} 1637$ is a very rapid rotator.

Using the $\mathrm{He}_{\mathrm{I}} \lambda 4471 / \mathrm{Mg}$ II $\lambda 4481$ line ratio found in the HIRES spectrogram of $\mathrm{BD}+65^{\circ} 1637$, we assess the temperature class to be $\sim \mathrm{B} 3$, consistent with classifications found in the literature. The HIRES spectrum reveals strong $\mathrm{H} \alpha$ emission ( $W=-25 \AA$ ) with wings extending out to at least $\pm 550 \mathrm{~km} \mathrm{~s}^{-1}$. There is no indication of an underlying, early-type stellar photosphere in the $\mathrm{H} \alpha$ emission profile. The emission line is double-peaked as shown in Figure 7 with radial velocities measured for the blueshifted and redshifted components of -60.5 and $+13.7 \mathrm{~km} \mathrm{~s}^{-1}$, respectively. The radial velocity of the core absorption feature is $-29.8 \mathrm{~km} \mathrm{~s}^{-1}$. These velocities differ substantially from those found by Finkenzeller \& Jankovics (1984), $-5 \mathrm{~km} \mathrm{~s}^{-1}$ (core absorption) and -36 and $+45 \mathrm{~km} \mathrm{~s}^{-1}$ (blue and red emission peaks, respectively). $\mathrm{H} \beta$, shown in Figure 7, reveals a similar double-peaked emission structure, but is substantially weaker $(W=-2.4 \AA)$. The velocities of the double-peaked $\mathrm{H} \beta$ emission profile are -78.6 and $+30.1 \mathrm{~km} \mathrm{~s}^{-1}$, with the red emission component having a higher amplitude than the blue component. Unlike the $\mathrm{H} \alpha$ profile, broad absorption wings are present in $\mathrm{H} \beta . \mathrm{H} \gamma$ falls outside the coverage of the HIRES spectrum, but the wing of its redward absorption edge is evident on the bluest order. If $\mathrm{H} \gamma$ were in emission, it would be expected to be weak. 
Other features of interest in the HIRES spectrum of BD+65 1637 include P Cygni-like profiles of He I $\lambda 4922$ and $\lambda 5015$ (possibly contaminated by $\mathrm{Fe}$ II emission), and broad, doublepeaked emission profiles of $\mathrm{Fe}$ II $\lambda \lambda 5169,5316,6317$ and 6456. These iron features consistently exhibit higher amplitude red emission peaks relative to blue (similar to $\mathrm{H} \alpha$ and $\mathrm{H} \beta$ ). The $\mathrm{Na}$ I D lines $(\lambda \lambda 5889.95,5895.92)$ exhibit no trace of emission and the narrow interstellar absorption components have heliocentric radial velocities of $\sim-19.7 \mathrm{~km} \mathrm{~s}^{-1}$. In the local standard of rest, these velocities are consistent with being produced within the local spiral arm, no more than $1 \mathrm{kpc}$ distant using the galactic rotation curves calculated by Münch (1957). Diffuse interstellar bands (DIBs) are also evident in the spectrum near $\lambda \lambda 5780,5797,5825$, and 5850 .

The heliocentric radial velocity of $\mathrm{BD}+65^{\circ} 1637$ is difficult to measure given the very broad absorption profiles induced by rapid rotation and the presence of $\mathrm{Fe}$ II emission near select $\mathrm{He}_{\mathrm{I}}$ absorption features. Alecian et al. (2013) determined a $v \sin i$ for $\mathrm{BD}+65^{\circ} 1637$ of $278 \pm 27 \mathrm{~km} \mathrm{~s}^{-1}$ and a radial velocity of -26 $\pm 20 \mathrm{~km} \mathrm{~s}^{-1}$, consistent with the heliocentric radial velocity of the molecular cloud reported by Finkenzeller \& Jankovics (1984), $-23.5 \mathrm{~km} \mathrm{~s}^{-1}$. Using a handful of He $\mathrm{I}$ absorption lines, we estimate the radial velocity of $\mathrm{BD}+65^{\circ} 1637$ to be $-25.6 \mathrm{~km} \mathrm{~s}^{-1}$.

Hillenbrand et al. (1992) classify $\mathrm{BD}+65^{\circ} 1637$ as a Group III object, i.e., a Be star with substantially less infrared excess than sources associated with massive envelopes or circumstellar disks. The infrared excess exhibited by $\mathrm{BD}+65^{\circ} 1637$ likely results from free-free emission from hot circumstellar gas, comparable to classical Be stars. Its association with the star-forming region, however, implies that the star is young. In their mid and far-infrared Infrared Space Observatory (ISO) survey of Herbig AeBe stars, Ábrahám et al. (2000) found that $\mathrm{BD}+65^{\circ} 1637$ was weakly detected at $60 \mu \mathrm{m}$, contrary to expectations if indeed disk-free. If confirmed, Ábrahám et al. (2000) suggest that this $60 \mu \mathrm{m}$ emission could arise from cold dust associated with the star. Lorenzetti et al. (2003) mapped NGC 7129 using the Long Wave Spectrometer onboard ISO in [O I] 63 and $145 \mu \mathrm{m}$ and in [C $\left.\mathrm{C}_{\mathrm{II}}\right] 158 \mu \mathrm{m}$. The line emission is suggestive of two PDRs, one being illuminated by BD+65 1637 and the other by $\mathrm{LkH} \alpha 234$. In their Spitzer MIPS $24 \mu \mathrm{m}$ survey of the region, Muzerolle et al. (2004) report that while the photospheres of $\mathrm{BD}+65^{\circ} 1637$ and $\mathrm{BD}+65^{\circ} 1638$ were not detected directly, extended halos of warm dust are apparent around their positions. The MIPS observations appear to confirm that a halo of dust enshrouds these early-type stars on scales larger than an envelope/disk environment.

\section{2. $B D+65^{\circ} 1638$}

$\mathrm{BD}+65^{\circ} 1638$, was classified by Hubble (1922) as B3, and is among the earliest spectral type cluster members. Racine (1968) also assigned a B3 type and derived an absolute magnitude of $M_{V}=-2.0$, somewhat more luminous than a normal B3 zero age main sequence (ZAMS) star. The slightly greater distance adopted here would imply that the star is even more luminous.

Matthews et al. (2003) note that $\mathrm{BD}+65^{\circ} 1638$ is accompanied by a small $\mathrm{H}_{\mathrm{II}}$ region and that its temperature and luminosity place it near the birthline of a $\sim 6 M_{\odot}$ star using the evolutionary models of Palla \& Stahler (1993). Matthews et al. (2003) assign a spectral type of B2.5 based upon $1.4 \mathrm{GHz}$ continuum flux and the star's estimated excitation parameter.
The HIRES spectrogram of $\mathrm{BD}+65^{\circ} 1638$ shows a $\mathrm{He} \mathrm{I}$ $\lambda 4471 / \mathrm{Mg}$ II $\lambda 4481$ line ratio that is consistent with a B3 spectral type. The spectrum also reveals possible evidence for emission reversal within the core of the $\mathrm{H} \alpha$ absorption profile shown in Figure 8. The centroid of this weak feature has a velocity of about $-25.8 \mathrm{~km} \mathrm{~s}^{-1}$, which is consistent with the radial velocity of the molecular cloud. $\mathrm{H} \beta$ appears to be a normal absorption profile (Figure 8) as might be expected for an early-type stellar photosphere. No emission features attributed to $\mathrm{Fe}$ II are present in its spectrum as with $\mathrm{BD}+65^{\circ}$ 1637 and $\mathrm{LkH} \alpha 234$ (discussed below).

The measured radial velocities for the interstellar $\mathrm{Na}$ I $\mathrm{D}$ lines are -21.9 and $-21.3 \mathrm{~km} \mathrm{~s}^{-1}$; comparable to those measured for $\mathrm{BD}+65^{\circ} 1637$. The heliocentric radial velocity of the star measured from $\mathrm{Mg}$ II $\lambda 4481, \mathrm{He}_{\text {I }} \lambda \lambda 4387,4437,4471,4713$, $4921,5015,5575,5875$ and 6678 is estimated to be $\sim+8.1 \mathrm{~km} \mathrm{~s}^{-1}$. Given the large disparity in the radial velocity of $\mathrm{BD}+65^{\circ} 1638$ relative to the molecular cloud, it is likely that $\mathrm{BD}+65^{\circ} 1638$ is a spectroscopic binary. Follow-up high dispersion spectroscopy is needed for confirmation. Otherwise the optical spectrum of $\mathrm{BD}+65^{\circ} 1638$ is quite unremarkable, particularly if the star were lying on or near the stellar birthline.

\section{3. $L k H \alpha 234$}

The luminous Herbig Be star $\mathrm{LkH} \alpha 234$ was classified by Herbig (1960) as having a late-A spectral type with strong $\mathrm{H} \alpha$ emission and moderate intensity $\mathrm{H} \beta$ emission. Strom et al. (1972) classify the star as B5-B7 and noted that $\mathrm{H} \beta$ and $\mathrm{H} \gamma$ were in emission with sharp P Cygni-like components. Possible weak emission was also noted for $\mathrm{He}$ I $\lambda \lambda 4922,5015$, and the star was identified as a moderately slow rotator. More recently Hernández et al. (2004) assign a spectral type of B7 for $\mathrm{LkH} \alpha$ 234. The HIRES spectrum of the source obtained in 2004 reveals a $\mathrm{He}_{\mathrm{I}} \lambda 4471 / \mathrm{Mg}$ II $\lambda 4481$ line ratio that is consistent with a B8 spectral type.

$\mathrm{LkH} \alpha 234$ is embedded within the ridge of molecular gas that defines the northeastern edge of the evacuated cavity. From its placement above the ZAMS in the color-magnitude diagram, it is generally assumed that $\mathrm{LkH} \alpha 234$ is among the youngest of the massive stars in NGC 7129. Hillenbrand et al. (1992) classify it as a Group I object with substantial infrared excess beginning near $1 \mu \mathrm{m}$ and extending out beyond $100 \mu \mathrm{m}$.

The HIRES spectra of LkH $\alpha 234$ obtained in 1999 and 2004 are suggestive of active accretion with numerous metallic and forbidden transitions in emission as well as $\mathrm{H} \alpha$ and $\mathrm{H} \beta$ exhibiting complex emission structure. Shown in Figure 9 are the $\mathrm{H} \alpha$ profiles for $\mathrm{LkH} \alpha 234$ from 1999 to 2004. While of comparable strength $(W=-56.6 \AA)$, the profiles are significantly different in appearance with the 1999 spectrum exhibiting deep absorption in a P Cygni-like profile. A second narrow absorption core is present near line center, just blueward of the sharp-edged emission peak. This peak and its stepped redward slope are remarkably similar in the two observations. The $\mathrm{H} \alpha$ profiles possess broad wings that extend several hundred $\mathrm{km} \mathrm{s}^{-1}$ from the central wavelength. The blueward side of the 2004 emission peak shows a distinct, sharp drop-off that never declines below the continuum level (Figure 9). The $\mathrm{H} \alpha$ profile is suggestive of a wind moving outward from the central star. This is supported by strong, blueshifted $\left[\mathrm{O}_{\mathrm{I}}\right] \lambda \lambda 6300,6363$ emission with heliocentric radial velocities of about $-23 \mathrm{~km} \mathrm{~s}^{-1}$, as well as blueshifted [S II] $\lambda \lambda$ 6717, 6731 emission with comparable velocities. The radial 

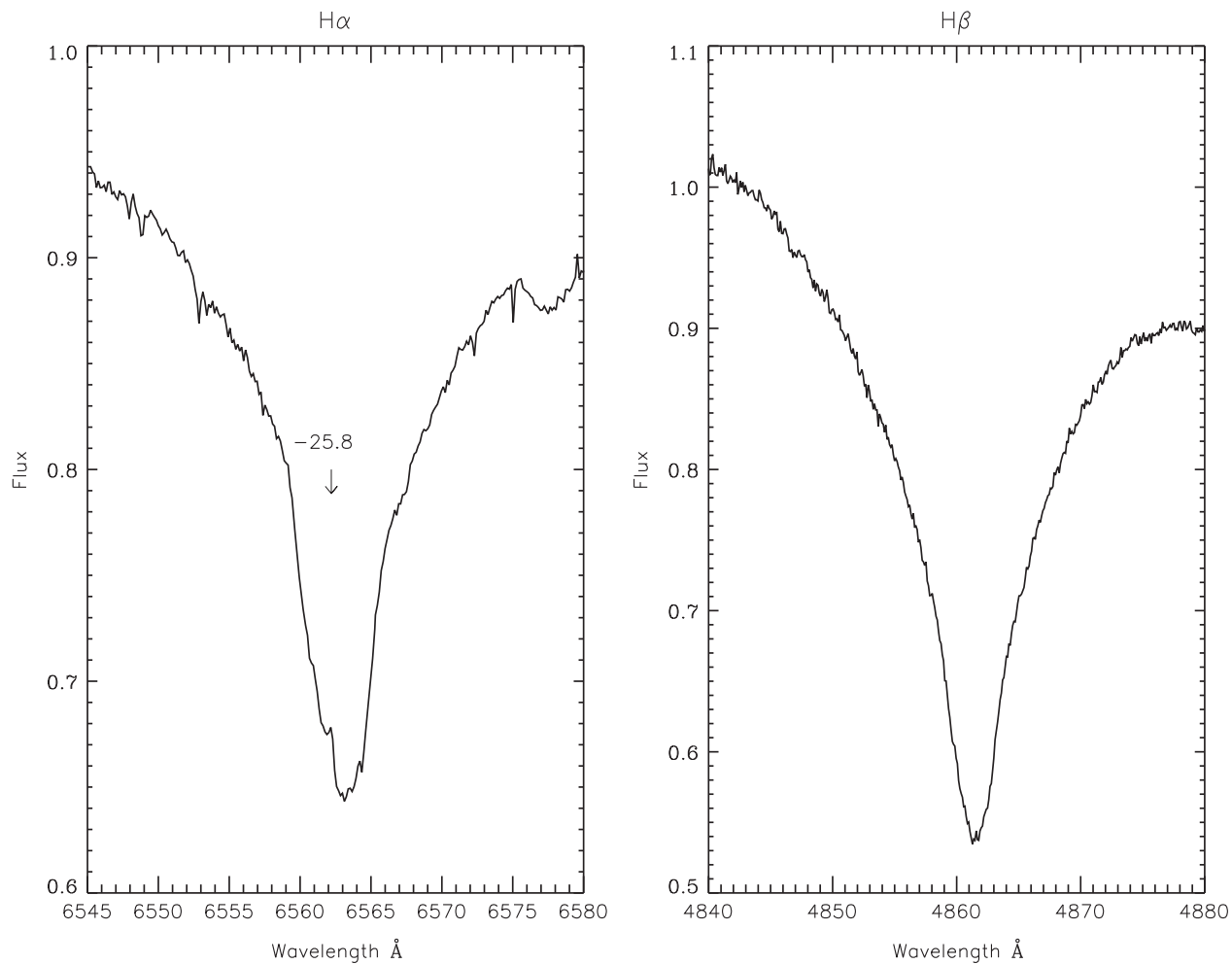

Figure 8. Sections of the HIRES spectrum of the $\mathrm{B} 3$ star $\mathrm{BD}+65^{\circ} 1638$ centered near $\mathrm{H} \alpha$ (left) and $\mathrm{H} \beta$ (right). The spectrum reveals weak evidence for possible emission reversal within the core of the $\mathrm{H} \alpha$ absorption feature with a radial velocity of $\sim-25.8 \mathrm{~km} \mathrm{~s}^{-1}$, consistent with that of the molecular cloud. Otherwise the spectrum of $\mathrm{BD}+65^{\circ} 1638$ is quite unremarkable in contrast to those of the classical Be star BD+65 1637 and the Herbig Be star LkH $\alpha 234$.

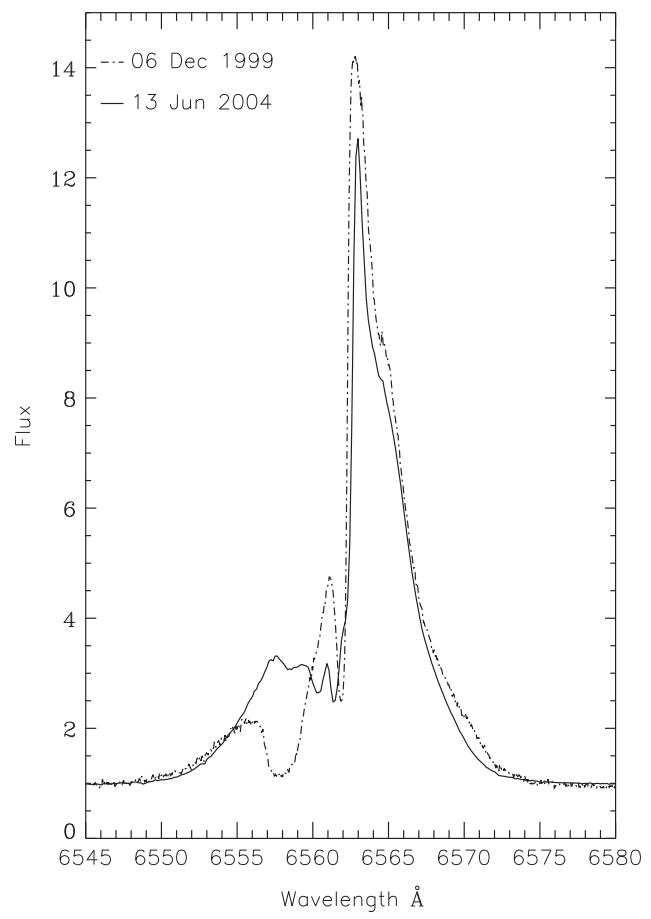

Figure 9. Sections of the HIRES spectra of LkH $\alpha 234$ obtained in 1999 December and 2004 June centered near $\mathrm{H} \alpha$ showing substantial differences in structure between the two epochs. The 1999 spectrum reveals a deep absorption feature in the P Cygni-like profile that is not evident in the later spectrum. A second narrow absorption core is evident near line center, just blueward of the sharp emission peak. The emission peak and the stepped redward slopes are remarkably similar in both observations. velocity of $\mathrm{LkH} \alpha 234$ could not be determined from the HIRES spectra as individual $\mathrm{He}_{\mathrm{I}}$ and $\mathrm{Mg}$ II absorption lines yield inconsistent results, possibly resulting from significant stellar activity.

Other prominent features in the spectrum of $\mathrm{LkH} \alpha 234$ include complex profiles of $\mathrm{Fe}$ II $\lambda \lambda 4922,5018$, which appear to possess broad emission bases with central absorption and core emission reversal rising well above the continuum level. The emission core has a radial velocity approximately equal to that of the other forbidden emission lines, implying that it is associated with an outward moving wind. The Na I D lines exhibit narrow and broad absorption components, the former with radial velocities of about $-20 \mathrm{~km} \mathrm{~s}^{-1}$ (interstellar) and the latter, $-83 \mathrm{~km} \mathrm{~s}^{-1}$. Shown in Figure 10 are the profiles of $\mathrm{H} \beta$, $\mathrm{Fe}_{\text {II }} \lambda 5018$, and the Na I D lines in the 2004 HIRES spectrum of $\mathrm{LkH} \alpha 234$. The radial velocities associated with various features in the spectrum are annotated for reference.

\subsection{SVS 13}

SVS 13 was previously unclassified in the literature, but the high dispersion spectroscopy presented here is suggestive of a mid- to-late B spectral type with very broad $\mathrm{He}$ I $\lambda \lambda 4921,5875$, 6678 absorption lines, implying rapid rotation. The profiles of $\mathrm{H} \beta$ and $\mathrm{H} \alpha$ are shown in Figure 11. While $\mathrm{H} \beta$ appears to be in pure absorption, $\mathrm{H} \alpha$ reveals a broad absorption profile with a narrow emission core having a heliocentric radial velocity of $-23 \mathrm{~km} \mathrm{~s}^{-1}$. Fe I $\lambda \lambda 4988,4994$ are in weak emission as is a broad [O I $] \lambda 6300$ feature. The Na I D lines exhibit no trace of emission and the narrow interstellar absorption components have heliocentric radial velocities of $\sim-19.5 \mathrm{~km} \mathrm{~s}^{-1}$, consistent 

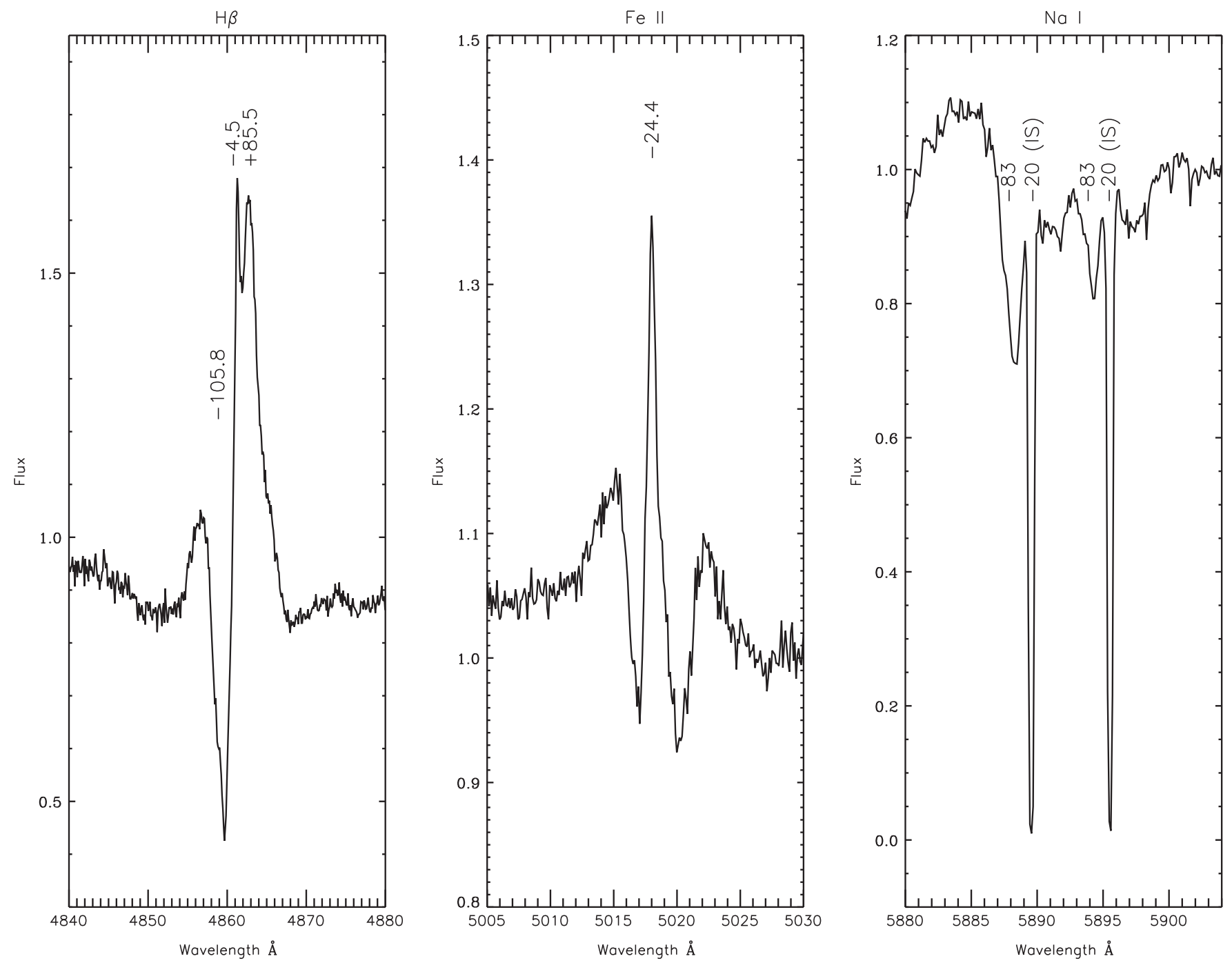

Figure 10. Sections of orders from the 2004 HIRES spectrum of $\mathrm{LkH} \alpha 234$ centered near $\mathrm{H} \beta$ (left panel), Fe II $\lambda 5018$ (center panel) and the Na I D lines (right panel). Radial velocities of various features in the spectra are annotated for reference.

with those found in the other early-type stars in the cluster. DIBs are also present near $\lambda \lambda 5780,5797$, and 5850 .

Stelzer \& Scholz (2009) identify SVS 13 as an X-ray source (S3-X51) and from its 2MASS and IRAC [3.6], [4.5] photometry, as a Class III infrared source, i.e., consistent with a stellar photosphere. Muzerolle et al. (2004), however, find the PSF core of SVS 13 in their MIPS $24 \mu \mathrm{m}$ imaging to be completely saturated. The $K$-band photometry presented here was obtained with the SQIID camera on the KPNO 50 inch telescope in 1993 (2MASS only provides a $K_{S}$ upper limit for this source). The star's placement in the near-infrared colorcolor diagrams to be discussed in Section 5.4 is consistent with circumstellar dust emission. If the observed infrared excess arises from a circumstellar disk, the $\mathrm{H} \alpha$ emission evident in the HIRES spectrum would be consistent with that of a Herbig Be star.

\subsection{V350 Cep}

The late-type (M2) pre-main sequence star V350 Cep lies near the edge of the L1181 molecular cloud, 5'.0 north of the cluster core. Around it lie several $\mathrm{H} \alpha$ emission sources as well as the bright infrared source SVS 10. Herbig (2008) provides an abridged history of V350 Cep observations, which include that the source was undetected on the 1954 Palomar Sky Survey plates, but rose to its current brightness $(V \sim 16)$ sometime in the late 1960s or early 1970s. The source is clearly visible on plate 1 of SVS, a red photograph of NGC 7129 obtained using the Mayall $4 \mathrm{~m}$ telescope at Kitt Peak. Pogosyants (1991) suggests that V350 Cep first exceeded the brightness limit $(V \sim 18.5)$ of archived photographic plates sometime during 1971. Following its apparent minimum, V350 Cep has now remained "bright" for over 40 years, and it remains unclear if this activity is repetitive as might be expected of an EXor.

Spectra of V350 Cep obtained by Magakyan \& Amirkhanyan (1979) suggest a T Tauri-like source, but were clearly variable as described by Magakyan (1983). Cohen \& Fuller (1985) found strong Balmer line emission as well as $[\mathrm{O} \mathrm{I}]$ and Fe II emission with an underlying M2-type photosphere, inferred from shallow absorption features in the red thought to arise from TiO bandheads. Hartigan \& Lada (1985) conclude that V350 Cep illuminates the small reflection nebula GGD 33 and may be an outflow source within the region. Goodrich (1986) obtained a low-resolution, spectrophotometric 

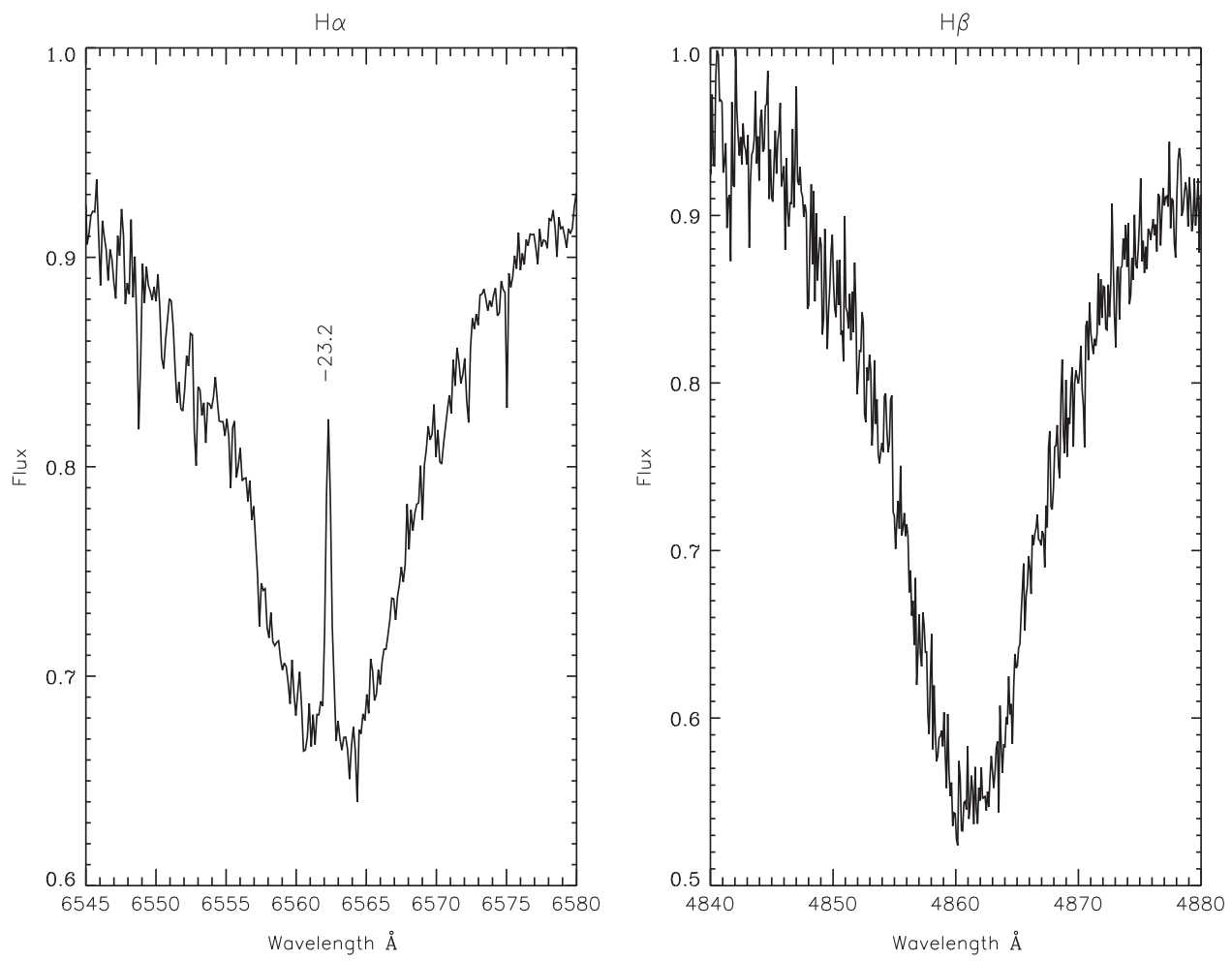

Figure 11. Sections of orders from the 2010 HIRES spectrum of SVS 13 centered near H $\alpha$ (left panel) and $\mathrm{H} \beta$ (right panel). The heliocentric radial velocity of the emission core centered within the broad $\mathrm{H} \alpha$ absorption profile is annotated. SVS 13 is possibly a Herbig Be star, but shows substantially less emission than either $\mathrm{LkH} \alpha 234$ or the classical Be star $\mathrm{BD}+65^{\circ} 1637$.

observation of V350 Cep and noted strong Balmer line emission as well as a rich Fe II emission spectrum.

The HIRES spectrum of V350 Cep presented here is described briefly by Herbig (2008), who otherwise focuses his discussion on an earlier 2005 spectrum, as exhibiting strong Balmer line emission as well as metallic line emission. The prominent metal lines are evident throughout the spectrum including Fe I $\lambda \lambda 6136,6191,6230$ (Figure 12); Fe II $\lambda \lambda 6317$, 6338,6456 . Strong He $\mathrm{I}$ emission is present including $\lambda \lambda 5875$, 6678. The Na I D lines (Figure 12) exhibit broad emission with narrow interstellar absorption components within their cores having radial velocities of $-18 \mathrm{~km} \mathrm{~s}^{-1}$, comparable to the velocities measured in the early-type stars. Broad Na I absorption features are also evident shifted some $-204 \mathrm{~km} \mathrm{~s}^{-1}$ blueward of the rest wavelength. Herbig (2008) suggests that these features originate from the same outflowing material responsible for the $\mathrm{P}$ Cygni feature in $\mathrm{H} \alpha$. Similar radial velocities were reported by Herbig (2008) for these broadened absorption features.

Forbidden emission, e.g., [O I] $\lambda 6300,[\mathrm{~N}$ III] $\lambda 6515,[\mathrm{~N}$ II $]$ $\lambda 6545,\left[\mathrm{O}_{\mathrm{II}}\right] \lambda 6589$, is present throughout the HIRES spectrum. Lithium $\lambda 6708$ is in absorption with an equivalent width of $\sim 0.10 \AA$, slightly reduced from that measured by Herbig (2008), $\sim 0.14 \AA$ in the earlier HIRES spectrum obtained in 2005. The heliocentric radial velocity of the star determined using a dozen metallic features in the $5700-6800 \AA$ wavelength region is $-20.7 \pm 0.9 \mathrm{~km} \mathrm{~s}^{-1}$, consistent with that measured by Herbig (1998) using the 2005 HIRES spectrum, $-23.0 \pm 0.3 \mathrm{~km} \mathrm{~s}^{-1}$.

$\mathrm{H} \alpha$ exhibits a strong P Cygni-like profile, $W=-27 \AA$ with a weak absorption feature lying within the core of the emission peak that is not evident in the 2005 spectrum obtained by
Herbig (2008). The deep P Cygni absorption structure at $\mathrm{H} \alpha$ has edges of -102 and $-252 \mathrm{~km} \mathrm{~s}^{1}$, defined at the continuum level. Figure 12 shows the $\mathrm{H} \alpha$ emission profile with the velocities annotated for various features. This figure can be directly compared with that shown in Figure 9 of Herbig (2008) from the 2005 HIRES spectrum. The overall shape of the line profile has changed somewhat while the equivalent width decreased only slightly.

Herbig (2008) concludes that V350 Cep is not an EXor candidate, i.e., a T Tauri-like star that undergoes periodic flareups presumably as the result of an inflow of material from the surrounding accretion disk. Muzerolle et al. (2004) classified V350 Cep as a Class II source from its placement in the Spitzer [3.6]-[5.8], [8]-[24] color-color diagram. While eliminated as an EXor candidate, V350 Cep is clearly undergoing significant accretion activity and is associated with a substantial circumstellar disk as inferred from its strong $\mathrm{H} \alpha$ emission and infrared excess. The nature of it dramatic rise from obscurity is worthy of additional study.

\section{NGC 7129: THE CLUSTER POPULATION}

\subsection{The Early-type Stars}

The four central, early-type stars $\mathrm{BD}+65^{\circ} 1638, \mathrm{BD}+65^{\circ}$ 1637, $\mathrm{LkH} \alpha 234$, and SVS 13 are the most prominent members of the emerging young cluster in NGC 7129. In addition to these sources, however, are approximately nine other bright stars in the region, dispersed over several arcminutes on sky.

On the southwestern edge of the evacuated cavity lie BD $+65^{\circ} 1635$ (SVS 5) and BD+65 1636 . The former is a G8-type star with measured proper motion and a probable field interloper, while the latter is a B8-type X-ray source 

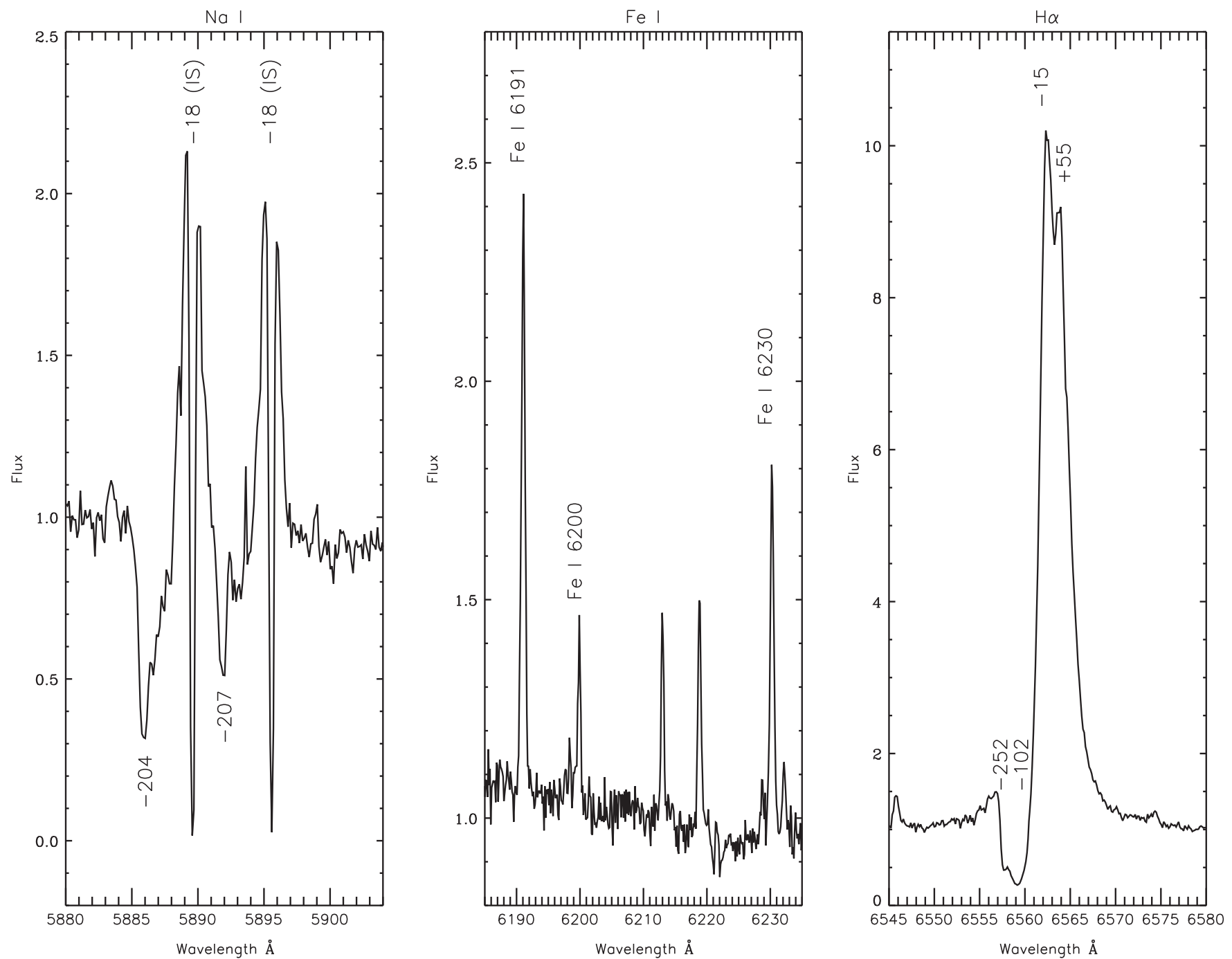

Figure 12. Sections of the HIRES spectrum of the enigmatic M2-type star V350 Cep centered near the Na I D lines (left panel), the numerous metallic emission lines near $\lambda 6220$ (center panel), and the P Cygni-like profile of $\mathrm{H} \alpha$ (right panel). Radial velocities of various features are indicated in the panels for reference.

presumably associated with the young cluster. Six arcminutes northwest of $\mathrm{BD}+65^{\circ} 1638$ lies $\mathrm{BD}+65^{\circ} 1631$, a K0-type giant lying in the foreground. Along a similar axis, 3.5 distant from $\mathrm{BD}+65^{\circ} 1638$, lies SVS 4, a field G-type giant. Several arcminutes north of the cluster, three moderately bright nebulous stars form an arc terminating near V350 Cep: SVS 2 (A1), SVS 10 (B8) and SVS 11. All of these sources are probable pre-main sequence stars that appear to be involved with the nebulosity. To the northeast, about six arcminutes from the cluster core, lies 2MASS J21435035+6608477, classified by Straižys et al. (2014) as a B7 dwarf. This source appears to illuminates a small reflection nebula on the edge of the molecular cloud. Finally, four arcminutes southeast of BD $+65^{\circ} 1638$ is SVS 16, a G6-type star and X-ray source that exhibits an unremarkable absorption line spectrum. This source appears to lie in the foreground of the molecular cloud, and its membership in the cluster remains unconfirmed.

In addition to these, the intermediate mass $\left(\sim 2-8 M_{\odot}\right)$ Class 0 source FIRS 2, is still embedded within its natal envelope south of the cluster core and will likely emerge as a mid-B type star (Fuente et al. 2014). In summary, there are at least four other B-type stars that are associated with NGC 7129 and the molecular cloud complex.

\subsection{The Ho Emission Sources}

In his landmark study of Be and Ae stars associated with nebulosity, Herbig (1960) reported that both BD+65 1637 and $\mathrm{LkH} \alpha 234$ exhibit $\mathrm{H} \alpha$ emission, but also noted the presence of several faint stars having $\mathrm{H} \alpha$ emission near the limit of the slitless grating exposures within the nebulosity of NGC 7129. From $R / \mathrm{H} \alpha$ magnitude ratios, Hartigan \& Lada (1985) found that in addition to $\mathrm{LkH} \alpha 234$ and $\mathrm{BD}+65^{\circ} 1637$, their sources $19 \mathrm{~S}, 14 \mathrm{~S}, 26 \mathrm{~S}, 13 \mathrm{~N}$ (V350 Cep), $19 \mathrm{~N}$, and $27 \mathrm{~N}$ were possible emitters. Several $\mathrm{HH}$ objects were also either confirmed or identified from the $\mathrm{H} \alpha$ imaging including GGD 32, 34 and 35, HH 103 and HH 105. Hartigan \& Lada (1985) suggested that $\mathrm{LkH} \alpha 234$ is the driving source for at least some of these $\mathrm{HH}$ objects. Magakian et al. (2004) identified 22 emission-line sources in NGC 7129, 16 of which were previously unknown. G. H. Herbig provided Magakian et al. (2004) with a list of $\mathrm{H} \alpha$ emission sources identified on an earlier WFGS image (not available for this analysis). Of 13 sources identified by Herbig as exhibiting $\mathrm{H} \alpha$ emission, five were not found in emission by Magakian et al. (2004), while their source MMN 11 was not identified by Herbig as an emitter. The WFGS images examined here reveal what appears 

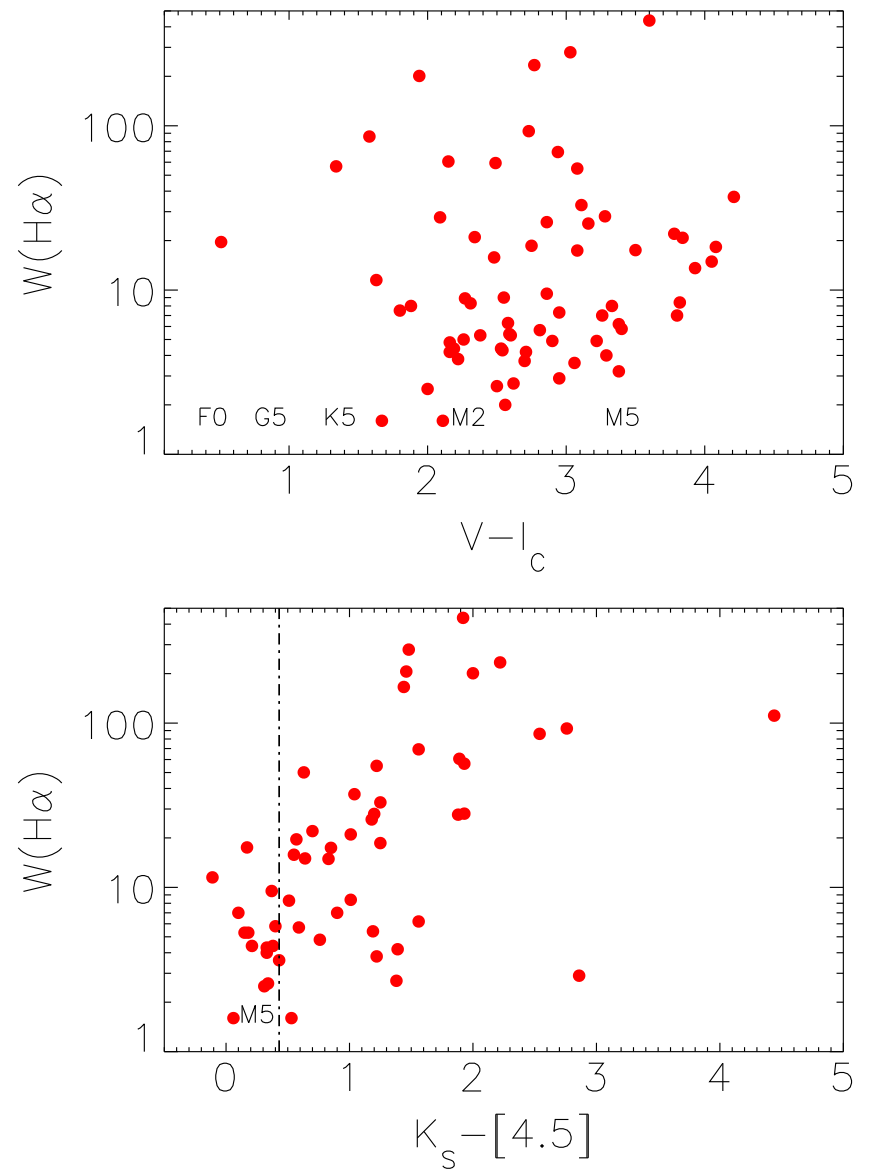

Figure 13. (Top panel) $V-I_{C}$ color plotted against $W(\mathrm{H} \alpha)$ for the $\mathrm{H} \alpha$ emission sources listed in Table 1. For reference the colors of 5-30 Myr stars are plotted along the abscissa. No obvious correlation is present, but the colors shown here have not been corrected for extinction. (bottom panel) $K_{S}-$ [4.5] color plotted as a function of $W(\mathrm{H} \alpha)$ for the $\mathrm{H} \alpha$ emission sources identified here. The reddest color expected from an unreddened stellar photosphere of M5 spectral type is indicated by the broken vertical line. A log-linear relationship is apparent between these two accretion disk parameters.

to be a flat continuum for MMN 11 (i.e., no absorption or emission), consistent with Herbig's findings. Of the 22 emission-line stars, Magakian et al. (2004) found that about half were concentrated in the central region of the reflection nebula. Ten of their sources were confidently identified as CTTS and another seven as weak-line T Tauri stars (WTTS).

The $\mathrm{H} \alpha$ slitless grism survey presented here identified over $50 \mathrm{H} \alpha$ emission sources in a region some $150 \operatorname{arcmin}^{2}$ in area, which is outlined in Figure 2. The HYDRA low-dispersion spectroscopy revealed $\sim 30$ additional emitters, most of which lie outside the boundaries of the WFGS survey, have measured equivalent widths below the detection threshold of the WFGS $(\sim 2 \AA)$, or had weak continua on the WFGS images. The positions of the more than $80 \mathrm{H} \alpha$ emission sources identified here are shown in Figure 2.

In Table 1 ordered by R.A., we present identifiers for the detected $\mathrm{H} \alpha$ emission sources, J2000 coordinates, spectral types, $V$-band magnitudes, $V-R_{C}$ and $V-I_{C}$ colors; $J-H$ and $H-K_{S}$ colors, and $K_{S}$ magnitudes from 2MASS; [3.6], [4.5], [5.8], and [8.0] Spitzer IRAC photometry from Stelzer \& Scholz (2009) and references therein as well as Wide-field Infrared Survey Explorer (WISE) $w 1, w 2, w 3$, and $w 4$ photometry. The WISE images of each source were examined individually in all passbands to ensure the sources were valid detections, particularly in the confusion-limited cluster center where nebulosity dominates infrared emission. For the previously unknown emission-line sources, an IH $\alpha$ number is assigned, continuing the numbering convention initiated by Herbig (1998) in IC 348 and subsequently continued by Herbig \& Dahm (2002) in IC 5146, Herbig et al. (2004) in NGC 1579, Dahm \& Simon (2005) in NGC 2264, Dahm (2005) in NGC 2362, Herbig \& Dahm (2006) in L988, and Dahm et al. (2012) in IC 1274. The measured equivalent widths of the $\mathrm{H} \alpha$ emission profiles, $W(\mathrm{H} \alpha)$, and of $\mathrm{Ca}$ II $\lambda 8542$ are also provided in Table 1 (positive values indicate emission). No corrections have been applied to $W(\mathrm{H} \alpha)$ or $W(8542)$ for underlying absorption structure.

The equivalent width of $\mathrm{H} \alpha$ is a well-established indicator of accretion processes and chromospheric activity in pre-main sequence stars (for a review, see Bertout 1989). Traditionally the boundary separating classical and WTTS was placed at $W$ $(\mathrm{H} \alpha)=10 \AA$ (e.g., Herbig 1998). While no physical interpretation was intended for this value, clear differences in the processes responsible for emission have since been recognized for CTTS, i.e., accretion, and WTTS, i.e., enhanced chromospheric activity. Various spectral type dependent criteria have been suggested to better distinguish accretors from nonaccretors, e.g., Martín (1998) and White \& Basri (2003). In Figure 13 we plot $W(\mathrm{H} \alpha)$ as a function of $V-I_{C}$ color (top panel) and $W(\mathrm{H} \alpha)$ as a function of $K_{S}-$ [4.5] color (bottom panel). If Spitzer [4.5] photometry were not available for a given source, $K_{S}-w 2$ values are substituted. While no obvious correlation is present between $W(\mathrm{H} \alpha)$ and $V-I_{C}$ color, a log-linear relationship is evident when comparing $W(\mathrm{H} \alpha)$ and $K_{S}-[4.5]$ color, both disk indicators. The normal $K-w 2$ color for young, 5-30 Myr M5-type stars (the latest spectral identified in the cluster, with one exception) is 0.43 mag (Pecaut \& Mamajek 2013). Sources lying redward of this value in Figure 13 should be considered as strong disk candidates.

The primary sources of contamination among the $\mathrm{H} \alpha$ emission sources are active late-K and M-type field dwarfs (dMe) that exhibit enhanced chromospheric activity. $\mathrm{H} \alpha$ emission strengths among dMe stars, however, are typically weak, $W(\mathrm{H} \alpha)<10 \AA$ (Hodgkin et al. 1995; Reid et al. 1995; Hawley et al. 1996), and would predominantly affect the statistics of the WTTS population. Other potential sources of contamination include chromospherically active giants, RS CVn binaries, cataclysmic variables, and active galaxies. The field density of such objects is expected to be low, particularly within the molecular clouds where extinctions reach $A_{V}$ 15-20 mag. The dark nebulosity likely obscures background sources over a substantial fraction of the survey area. With these caveats we assume that field contamination among the $\mathrm{H} \alpha$ emission sources is low, but certainly non-zero.

\subsection{Chandra ACIS X-ray Detected Sources}

Stelzer \& Scholz (2009) obtained a $22 \mathrm{ks}$ long integration of NGC 7129 using ACIS onboard Chandra, detecting 59 Xray sources. The majority (47/59) of these sources have 2MASS near-infrared counterparts, but prior to this survey few had optical photometry available. Correlating these Xray detections with 2MASS and Spitzer infrared excess sources, Stelzer \& Scholz (2009) identified one Class 0/I source, 16 Class II sources, and 30 Class III candidates, leaving 12 unclassified. Contamination of the X-ray selected 
sample from field interlopers, particularly among the Class III sources was considered, however, using X-ray luminosities of field dwarfs, Stelzer \& Scholz (2009) estimate that $<10^{-5}$ $\mathrm{X}$-ray emitting field stars lie within the central core of the cluster. A contribution of extragalactic X-ray sources was also considered, but reduced sensitivity at large off-axis angles should limit the total number of extragalactic sources considerably.

The X-ray detections from the Chandra ACIS integration are plotted as green crosses in Figure 2. Over half, 30, of the Xray detections have counterparts in the $\mathrm{H} \alpha$ emission selected sample. About a half-dozen X-ray detections in the halo of the star-forming region lacking optical counterparts are possible extragalactic sources. Others appear to be associated with stars that were not identified as $\mathrm{H} \alpha$ emitters, but that could be cluster members. Clearly X-ray and $\mathrm{H} \alpha$ emission are tracing similar activity in these pre-main sequence candidates. There are a substantial number of $\mathrm{H} \alpha$ emission sources, however, that were not detected by the X-ray survey, particularly off-axis. This is likely the result of the reduced sensitivity and the relatively shallow ACIS exposure. Included in Table 1, ordered by right ascension, are 46 of 59 X-ray sources identified by Stelzer \& Scholz (2009), their J2000 coordinates, spectral types, optical $\left(V, V-R_{C}, V-I_{C}\right)$ and infrared photometry from 2MASS $\left(J-H, H-K_{S}, K_{S}\right)$, Spitzer, and WISE. Of the remaining 13 sources, eight were outside of the fields of view of the optical photometric surveys and five had no optical counterparts.

\subsection{Infrared Excess Sources not Detected by

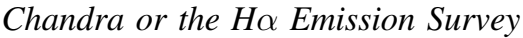

The Spitzer IRAC and MIPS photometry of Gutermuth et al. (2004) and Muzerolle et al. (2004) were used by Stelzer \& Scholz (2009) to identify sources with infrared excess emission attributable to circumstellar disks. Stelzer \& Scholz (2009) made their source selection using the [3.6] - [4.5], [5.8] - [8.0] and the $K_{S}-$ [4.5], $J-H$ colorcolor diagrams, resulting in the identification of 64 Class II sources and 13 Class 0/I candidate cluster members. Of these 77 sources, 46 were within the Chandra field of view, but were not detected in X-rays. Eliminating 15 of these sources that were detected with $\mathrm{H} \alpha$ emission and that already appear in Table 1, we list in Table 2 the remaining 31 pre-main sequence candidates that were selected on the basis of their infrared photometry. Contamination of these pre-main sequence candidates is dominated by extragalactic sources, predominantly star-forming galaxies. Such objects, however, reside in a specific region of the [3.6] - [4.5], [5.8] - [8.0] color-color diagram and can be distinguished from pre-main sequence stars by establishing a brightness cutoff determined by statistical means (Gutermuth et al. 2008; Stelzer \& Scholz 2009). In Table 2 we present optical $\left(I_{C}, R_{C}-I_{C}\right)$ photometry for these Spitzer excess sources and infrared photometry from 2MASS, Spitzer, and WISE. These sources are shown in Figure 2 as open red squares and appear to be preferentially positioned within the semi-circular arc of remnant molecular gas to north, east and south of the cluster core. The Class I source adjacent to $\mathrm{LkH} \alpha 234$ is $2 \mathrm{MASS}$ $\mathrm{J} 21430696+660641.7$, which is discussed in more detail in Section 6.2.

\subsection{Spectroscopically Classified Sources Lacking $\mathrm{H \alpha}$ or $\mathrm{X}$-ray Emission}

In Table 3 we present optical and infrared photometry from 2MASS and WISE for sources that were classified by the lowdispersion spectroscopic survey, but that lack $\mathrm{H} \alpha$ or detected $\mathrm{X}$-ray emission. In Section 5.4, the near-infrared color-color diagrams are used to identify several of these stars as possible infrared excess sources. The majority, however, are probable field interlopers, not associated with the young cluster or the molecular cloud complex, but are presented here for completeness.

\section{CLUSTER PROPERTIES}

\subsection{Reddening and Extinction}

Extinction and CO column density are in general strongly correlated, implying that dust is well-mixed with the molecular gas in molecular cloud complexes. Shown in Figure 14 is a map of visual extinction $\left(A_{V}\right)$ derived from ${ }^{13} \mathrm{CO}$ integrated line intensity obtained at the Five College Radio Astronomical Observatory (FCRAO) in 1993 by LAH. The map is superimposed upon a near-infrared mosaic of the region with extinction peaks that are coincident with the compressed ridge to the north, east and south of the cluster core, peaking near $A_{V}$ $\sim 20$ mag. The evacuated cavity is clearly evident with extinction dropping sharply to the west and gradually to the east across the surface of the molecular cloud. Also shown in Figure 14 is a map of integrated CS line intensity. The CS extinction map demonstrates significant enhancements near the molecular outflow originating by $\mathrm{LkH} \alpha 234$ and to the south near FIRS 2.

The low-dispersion spectroscopy allowed spectral classification for $\sim 130$ stars in the cluster region. Combined with the optical photometry, the spectral types permitted an independent determination of extinction across the region. To estimate extinction for sources of known spectral type, we assume the standard ratio of total-to-selective absorption, i.e., $R=A_{V} / E(B-V)=3.08$ (He et al. 1995) to derive a normal reddening law given by $A_{V}=2.43 E\left(V-I_{C}\right)$. Intrinsic colors of 5-30 Myr old pre-main sequence stars taken from Pecaut \& Mamajek (2013) were used to determine $V-I_{C}$ color excesses and extinctions. The average extinction suffered by 50 probable cluster members taken from the $\mathrm{H} \alpha$ and X-ray selected stellar samples is $A_{V}=1.8 \mathrm{mag}$. An abnormal extinction law induced by dust grains having sizes substantially larger than interstellar grains or the peculiar SEDs for these pre-main sequence members could impact this adopted mean extinction value.

\subsection{The Color-Magnitude Diagram of the Cluster Population}

Shown in Figure 15 is the observed $V-I_{C}, V$ colormagnitude diagram for all sources detected by the KPNO T2KA CCD imaging survey. The candidate members of NGC 7129, i.e., the $\mathrm{H} \alpha$ emission and X-ray detected sources, are superposed in the figure. The ZAMS of Siess et al. (2000) is overplotted using the dwarf colors presented by Kenyon \& Hartmann (1995) and assuming a distance of 1150 pc (Straižys et al. 2014). The cluster sequence is well-defined by the activity-selected sample and lies at least two magnitudes above the ZAMS, even for the early-type members.

The X-ray and $\mathrm{H} \alpha$ emission populations overlap considerably and appear to have identified the majority of possible 
Table 2

Photometry for Infrared Sources without X-ray or $\mathrm{H} \alpha$ Emission $^{\mathrm{a}}$

\begin{tabular}{|c|c|c|c|c|c|c|c|c|c|c|c|c|c|c|c|c|}
\hline Source & $\begin{array}{c}\alpha \\
(\mathbf{J} 2000)\end{array}$ & $\begin{array}{c}\delta \\
(\mathrm{J} 2000)\end{array}$ & $R_{C}-I_{C}{ }^{\mathrm{b}}$ & $I_{C}^{\mathrm{b}}$ & $J-H^{\mathrm{c}}$ & $H-K_{S}{ }^{\mathrm{c}}$ & $K_{S}^{\mathrm{c}}$ & {$[3.6]^{\mathrm{d}}$} & {$[4.5]^{\mathrm{d}}$} & {$[5.8]^{\mathrm{d}}$} & {$[8.0]^{\mathrm{d}}$} & $w 1^{\mathrm{e}}$ & $w 2^{\mathrm{e}}$ & $w 3^{\mathrm{e}}$ & $w 4^{\mathrm{e}}$ & Other Identifiers \\
\hline S3-U1178 & 214155.30 & +660741.5 & $\ldots$ & $\ldots$ & $\ldots$ & $\ldots$ & $\cdots$ & 15.86 & 14.90 & 13.98 & 12.78 & 16.10 & 15.12 & 12.15 & 8.99 & $\ldots$ \\
\hline S3-U1367 & 214217.67 & +660840.3 & $\cdots$ & $\cdots$ & 1.15 & 0.39 & 14.63 & 13.68 & 13.23 & 12.91 & 12.40 & 14.06 & 13.61 & $\cdots$ & $\cdots$ & $\cdots$ \\
\hline S3-U1194 & 214242.42 & +660745.2 & 2.42 & 18.62 & 1.35 & 0.78 & 13.45 & 12.56 & 12.21 & 12.23 & 11.68 & 12.07 & 11.80 & $\cdots$ & $\cdots$ & $\cdots$ \\
\hline S3-U1521 & 214242.86 & +660924.0 & $\ldots$ & $\ldots$ & $\ldots$ & $\ldots$ & $\ldots$ & 16.23 & 15.25 & 13.84 & 12.79 & $\cdots$ & $\ldots$ & $\cdots$ & $\cdots$ & $\cdots$ \\
\hline S3-U1246 & 214248.23 & +660800.6 & 2.30 & 18.16 & 1.40 & 0.81 & 13.08 & 11.69 & 11.14 & 10.62 & 9.67 & 11.23 & 10.71 & $\cdots$ & $\cdots$ & $\cdots$ \\
\hline S3-U722 & 214254.63 & +660520.3 & 2.66 & 18.50 & 0.95 & 0.69 & 13.87 & 12.51 & 12.64 & $\ldots$ & $\cdots$ & $\cdots$ & $\ldots$ & $\ldots$ & $\cdots$ & $\ldots$ \\
\hline S3-U546 & 214257.75 & +660423.5 & 1.08 & 18.00 & $\ldots$ & 0.82 & 13.66 & 11.80 & 10.94 & 9.95 & 8.54 & 11.85 & 10.74 & 7.10 & 5.07 & V392 Cep, RNO 138 \\
\hline S3-U1780 & 214259.47 & +66 1035.8 & $\ldots$ & $\ldots$ & $\ldots$ & 1.07 & 14.61 & 13.54 & 13.00 & 12.52 & 12.05 & 13.88 & 13.09 & $\cdots$ & $\cdots$ & $\cdots$ \\
\hline S3-U270 & 214259.82 & +660154.9 & $\ldots$ & $\ldots$ & $\ldots$ & $\ldots$ & $\ldots$ & 16.83 & 16.59 & 15.91 & 14.33 & $\cdots$ & $\cdots$ & $\cdots$ & $\cdots$ & $\cdots$ \\
\hline S3-U419 & 214301.78 & +660324.4 & $\cdots$ & $\cdots$ & $\ldots$ & $\ldots$ & $\cdots$ & 13.02 & 10.41 & 10.17 & 9.08 & 13.17 & 9.86 & 6.03 & 0.36 & FIRS 2 \\
\hline S3-U500 & 214302.01 & +660402.7 & $\cdots$ & $\cdots$ & $\ldots$ & $\cdots$ & $\cdots$ & 13.64 & 12.98 & 12.18 & 11.29 & 13.37 & 12.32 & $\cdots$ & $\cdots$ & $\cdots$ \\
\hline S3-U1611 & 214302.62 & +660950.7 & 2.60 & 18.08 & 0.92 & 0.59 & 14.20 & 13.54 & 13.16 & 12.65 & 11.93 & 13.92 & 13.32 & $\cdots$ & $\cdots$ & $\cdots$ \\
\hline S3-U1294 & 214302.89 & +66 0814.2 & $\cdots$ & $\cdots$ & 0.92 & 0.41 & 15.01 & 14.15 & 13.83 & 13.58 & 13.43 & $\cdots$ & $\cdots$ & $\cdots$ & $\cdots$ & $\cdots$ \\
\hline S3-U822 & 214304.38 & +66 0556.4 & $\ldots$ & 18.55 & 0.58 & 0.65 & 14.47 & $\ldots$ & 12.55 & $\ldots$ & $\ldots$ & $\ldots$ & $\ldots$ & $\ldots$ & $\ldots$ & $\ldots$ \\
\hline S3-U1242 & 214305.92 & +660758.5 & $\ldots$ & $\ldots$ & $\ldots$ & $\ldots$ & $\ldots$ & 13.78 & 12.70 & 12.10 & 11.65 & $\ldots$ & $\ldots$ & $\ldots$ & $\ldots$ & $\ldots$ \\
\hline S3-U968 & 214306.96 & +660641.7 & $\ldots$ & $\ldots$ & $\ldots$ & 2.34 & 10.89 & 7.54 & 6.59 & 5.67 & 5.06 & 8.38 & 6.75 & 4.15 & 0.70 & $\mathrm{~J} 21430696+660641.7$ \\
\hline S3-U1103 & 214307.84 & +660718.5 & 2.42 & 19.38 & 1.87 & 0.91 & 13.94 & 13.04 & 12.57 & 11.76 & 10.88 & $\cdots$ & $\cdots$ & $\ldots$ & $\ldots$ & $\ldots$ \\
\hline S3-U550 & 214311.17 & +660425.6 & $\cdots$ & $\cdots$ & $\cdots$ & $\cdots$ & $\cdots$ & 13.91 & 13.42 & 12.98 & 12.33 & 13.83 & 13.11 & $\cdots$ & $\cdots$ & $\cdots$ \\
\hline S3-U1026 & 214312.42 & +660655.9 & $\cdots$ & $\cdots$ & $\cdots$ & $\cdots$ & $\cdots$ & 14.16 & 13.73 & 13.25 & 12.23 & $\cdots$ & $\cdots$ & $\cdots$ & $\cdots$ & $\cdots$ \\
\hline S3-U1211 & 214314.17 & +660746.5 & $\cdots$ & $\cdots$ & $\cdots$ & $\cdots$ & 14.34 & 10.55 & 9.34 & 8.41 & 7.51 & $\cdots$ & $\cdots$ & $\cdots$ & $\cdots$ & $\cdots$ \\
\hline S3-U1169 & 214314.83 & +660737.5 & $\cdots$ & $\cdots$ & $\ldots$ & $\cdots$ & 14.77 & 12.93 & 10.72 & 10.44 & 10.22 & $\cdots$ & $\cdots$ & $\cdots$ & $\cdots$ & $\ldots$ \\
\hline S3-U1350 & 214324.13 & +660831.5 & $\cdots$ & $\cdots$ & $\cdots$ & 1.43 & 14.19 & 12.86 & 11.28 & 10.05 & 8.96 & 13.07 & 11.04 & 8.85 & 4.64 & GGD $34 \mathrm{C}$ \\
\hline S3-U1059 & 214324.90 & +660704.7 & 1.58 & 19.72 & $\cdots$ & $\cdots$ & $\cdots$ & 14.23 & 13.33 & 12.59 & 11.56 & $\cdots$ & $\ldots$ & $\ldots$ & $\ldots$ & $\ldots$ \\
\hline S2-U1313 & 214326.64 & +660820.5 & $\cdots$ & $\cdots$ & $\cdots$ & $\cdots$ & $\cdots$ & 14.66 & 14.19 & 13.79 & 13.24 & 14.97 & 13.92 & $\cdots$ & $\cdots$ & $\cdots$ \\
\hline S3-U821 & 214329.32 & +660555.7 & 2.70 & 18.46 & 0.92 & 0.57 & 14.39 & 13.47 & 13.04 & 12.52 & 11.02 & 13.71 & 13.07 & $\cdots$ & $\cdots$ & $\cdots$ \\
\hline S2-U1083 & 214329.92 & +660709.2 & $\cdots$ & 21.79 & $\cdots$ & $\cdots$ & $\cdots$ & 14.67 & 14.24 & 13.99 & 13.38 & $\cdots$ & $\cdots$ & $\cdots$ & $\cdots$ & $\cdots$ \\
\hline S2-U804 & 214333.12 & +660549.0 & $\ldots$ & $\ldots$ & $\ldots$ & $\ldots$ & $\ldots$ & 16.69 & 15.83 & 14.92 & 13.37 & $\ldots$ & $\ldots$ & $\ldots$ & $\ldots$ & $\ldots$ \\
\hline S2-U2219 & 214338.59 & +66 1230.6 & $\cdots$ & $\cdots$ & $\ldots$ & $\ldots$ & $\ldots$ & 13.58 & 13.34 & 12.80 & 11.99 & 13.42 & 13.06 & $\cdots$ & $\cdots$ & $\cdots$ \\
\hline S2-U1660 & 214347.04 & +66 1001.2 & 1.11 & 17.04 & 0.70 & 0.51 & 14.19 & 14.05 & 14.03 & 13.72 & 13.00 & 13.84 & 13.82 & $\ldots$ & $\cdots$ & $\cdots$ \\
\hline S2-U613 & 214348.70 & +66 0446.2 & $\cdots$ & & 1.23 & 0.57 & 13.08 & 12.40 & 12.15 & 11.83 & 10.87 & 12.33 & 11.88 & 9.65 & $\ldots$ & $\cdots$ \\
\hline S2-U1713 & 214349.35 & +661012.9 & 1.24 & 17.79 & 0.79 & 0.21 & 14.86 & 14.53 & 14.47 & 14.29 & 13.36 & 14.33 & 14.53 & & $\cdots$ & $\cdots$ \\
\hline
\end{tabular}

Notes.

${ }^{\text {a }}$ Young Stellar Objects identified by Stelzer \& Scholz (2009) based upon infrared excess emission.

${ }^{\mathrm{b}}$ Optical photometry from Keck LRIS imaging, UH $2.2 \mathrm{~m}$ or the KPNO $0.9 \mathrm{~m}$.

${ }^{c}$ Near-infrared photometry from the 2MASS Point Source Catalog.

d Spitzer mid-infrared photometry from Stelzer \& Scholz (2009).

${ }^{\mathrm{e}}$ Mid-infrared photometry from the AllWISE Source Catalog. 
Table 3

Photometry for Spectroscopically Classified Stars without X-ray or $\mathrm{H} \alpha$ Emission

\begin{tabular}{|c|c|c|c|c|c|c|c|c|c|c|c|c|c|c|}
\hline Source $^{a}$ & $\begin{array}{c}\alpha \\
(\mathrm{J} 2000)\end{array}$ & $\begin{array}{c}\delta \\
(\mathrm{J} 2000)\end{array}$ & SpT & $V^{\mathrm{b}}$ & $V-R_{C}^{\mathrm{b}}$ & $R_{C}-I_{C}^{\mathrm{b}}$ & $J-H^{\mathrm{c}}$ & $H-K_{S}{ }^{\mathrm{c}}$ & $K_{S}^{\mathrm{c}}$ & $w 1^{\mathrm{d}}$ & $w 2^{\mathrm{d}}$ & $w 3^{\mathrm{d}}$ & $w 4^{\mathrm{d}}$ & Comments \\
\hline 1 & 214222.75 & +660752.9 & G3-5 & 16.65 & 0.82 & 0.75 & 0.54 & 0.22 & 13.25 & 13.06 & 13.14 & $\ldots$ & $\ldots$ & $\ldots$ \\
\hline 2 & 214222.85 & +660656.3 & G-K1 & 20.57 & 1.34 & 1.11 & 0.80 & 0.55 & 14.90 & $\ldots$ & 14.06 & $\ldots$ & $\ldots$ & IR excess \\
\hline 3 & 214223.07 & +660642.5 & $\mathrm{~K} 1-3$ & 18.86 & 1.04 & 0.87 & 0.66 & 0.13 & 14.59 & $\ldots$ & $\ldots$ & $\ldots$ & $\ldots$ & $\ldots$ \\
\hline 4 & 214223.31 & +6608 47.4 & M0 & 17.45 & 1.01 & 0.93 & 0.68 & 0.21 & 13.45 & 13.35 & 13.35 & $\ldots$ & $\ldots$ & $\ldots$ \\
\hline 5 & 214224.74 & +660621.4 & F9-G5 & 17.59 & 1.23 & 1.14 & 0.88 & 0.32 & 12.22 & 11.72 & 11.88 & $\ldots$ & $\ldots$ & $\ldots$ \\
\hline 6 & 214225.19 & +661009.0 & F7-G5 & 18.14 & 0.96 & 0.91 & 0.42 & 0.32 & 14.15 & 13.99 & 13.92 & $\ldots$ & $\ldots$ & $\ldots$ \\
\hline 7 & 214226.84 & +660742.5 & G9-K0 & 10.59 & 0.57 & 0.50 & 0.55 & 0.07 & 7.96 & 7.84 & 7.93 & 7.68 & $\ldots$ & SVS 4 \\
\hline 8 & 214228.35 & +660408.7 & F5-9 & 18.41 & 0.99 & 0.95 & 0.62 & 0.22 & 14.18 & 13.43 & 13.66 & $\ldots$ & $\ldots$ & IR excess \\
\hline 9 & 214231.22 & +660804.8 & G9-K1 & 19.17 & 1.01 & 0.91 & 0.52 & 0.29 & 14.98 & 14.55 & $\ldots$ & $\ldots$ & $\ldots$ & $\ldots$ \\
\hline 10 & 214231.86 & +660708.7 & G6-8 & 17.29 & 0.99 & 0.95 & 0.61 & 0.18 & 13.11 & 12.53 & 12.71 & $\ldots$ & $\ldots$ & $\ldots$ \\
\hline 11 & 214232.57 & +661024.9 & G8-K0 & 18.54 & 1.02 & 0.96 & 0.69 & 0.16 & 14.26 & 14.07 & 14.23 & $\ldots$ & $\ldots$ & $\ldots$ \\
\hline 12 & 214233.75 & +660802.3 & M2.5 & 19.93 & 1.69 & 1.55 & 0.93 & 0.40 & 13.37 & 12.98 & 13.03 & $\ldots$ & $\ldots$ & $\ldots$ \\
\hline 13 & 214234.28 & +660312.6 & G-K4 & 18.37 & 1.20 & 0.92 & 0.79 & 0.23 & 13.73 & 13.42 & 13.59 & $\ldots$ & $\ldots$ & $\ldots$ \\
\hline 14 & 214235.03 & +660409.6 & G5-K0 & 18.36 & 0.82 & 0.77 & 0.52 & 0.23 & 14.90 & 14.10 & 14.99 & $\ldots$ & $\ldots$ & $\ldots$ \\
\hline 15 & 214235.69 & +660549.3 & K3 & 17.44 & 1.05 & 0.95 & 0.74 & 0.32 & 12.80 & 12.77 & 13.02 & $\ldots$ & $\ldots$ & $\ldots$ \\
\hline 16 & 214238.58 & +660941.4 & M2.5 & 20.27 & 1.35 & 1.32 & 0.50 & 0.70 & 15.10 & 14.39 & 14.26 & $\ldots$ & $\ldots$ & IR excess \\
\hline 17 & 214239.05 & +660709.9 & K5 & 18.37 & 1.67 & 1.53 & 1.09 & 0.44 & 11.08 & 10.46 & 10.39 & $\ldots$ & $\ldots$ & IR excess \\
\hline 18 & 214241.26 & +660333.7 & G2-5 & 16.02 & 0.77 & 0.74 & 0.52 & 0.12 & 12.80 & 12.66 & 12.73 & 12.27 & $\ldots$ & $\ldots$ \\
\hline 19 & 214244.96 & +660748.0 & G2-K & 22.03 & 2.06 & 1.57 & 1.03 & 0.48 & 14.06 & $\ldots$ & $\ldots$ & $\ldots$ & $\ldots$ & $\ldots$ \\
\hline 20 & 214245.98 & +660815.6 & K5 & 20.49 & 1.68 & 1.49 & 1.03 & 0.52 & 13.44 & 12.57 & 12.49 & $\ldots$ & $\ldots$ & IR excess \\
\hline 21 & 214246.02 & +661020.3 & K5: & 22.69 & 2.18 & 2.21 & 1.11 & 0.65 & 13.34 & 12.79 & 12.39 & $\cdots$ & $\ldots$ & IR excess \\
\hline 22 & 214247.73 & +660535.2 & G2-5 & 17.86 & 0.97 & 0.86 & 0.64 & 0.08 & 13.86 & 12.84 & 12.54 & $\ldots$ & $\ldots$ & IR excess \\
\hline 23 & 214250.66 & +660331.3 & G9-K2 & 16.95 & 0.73 & 0.58 & 0.61 & 0.17 & 13.99 & 13.88 & $\ldots$ & $\cdots$ & $\ldots$ & $\ldots$ \\
\hline 24 & 214250.47 & +660832.7 & K2-5 & 21.26 & 1.53 & 1.37 & 1.32 & 0.31 & 14.74 & $\ldots$ & $\ldots$ & $\ldots$ & $\ldots$ & $\ldots$ \\
\hline 25 & 214251.16 & +660545.3 & F2 & 17.29 & 0.59 & 0.58 & 0.26 & -0.03 & 14.90 & $\ldots$ & $\ldots$ & $\ldots$ & $\ldots$ & $\ldots$ \\
\hline 26 & 214251.94 & +660944.9 & M1 & 18.05 & 1.03 & 0.91 & 0.70 & 0.23 & 13.93 & 13.97 & $\ldots$ & $\ldots$ & $\ldots$ & $\ldots$ \\
\hline 27 & 214253.97 & +6608 15.1 & G5-K0 & 19.80 & 1.57 & 1.41 & 1.17 & 0.43 & 12.82 & $\ldots$ & $\ldots$ & $\ldots$ & $\ldots$ & $\ldots$ \\
\hline 28 & 214255.18 & +660625.4 & K5 & 15.83 & 0.84 & 0.68 & 0.68 & 0.15 & 12.53 & $\ldots$ & $\ldots$ & $\ldots$ & $\ldots$ & $\ldots$ \\
\hline 29 & 214258.04 & +660554.7 & G3-5 & 14.21 & 0.41 & 0.40 & 0.31 & 0.20 & 12.26 & $\ldots$ & $\ldots$ & $\ldots$ & $\ldots$ & $\ldots$ \\
\hline 30 & 214300.20 & +660546.1 & G8 & 17.47 & 0.76 & 0.65 & 0.84 & 0.01 & 13.87 & $\ldots$ & $\ldots$ & $\ldots$ & $\ldots$ & $\ldots$ \\
\hline 31 & 214305.32 & +660452.0 & M1 & 19.57 & 1.09 & 1.11 & 0.74 & 0.30 & 14.93 & $\ldots$ & $\ldots$ & $\ldots$ & $\ldots$ & $\ldots$ \\
\hline 32 & 214305.05 & +66 1012.4 & M1 & 20.97 & 1.37 & 1.51 & 1.02 & 0.48 & 14.14 & 14.07 & 14.03 & $\ldots$ & $\ldots$ & $\ldots$ \\
\hline 33 & 214305.53 & +660328.7 & K5 & 16.93 & 0.86 & 0.66 & 0.72 & 0.07 & 13.63 & 13.41 & 13.45 & $\ldots$ & $\ldots$ & $\ldots$ \\
\hline 34 & 214307.51 & +660901.5 & M3 & 20.84 & 1.85 & 1.72 & 1.12 & 0.36 & 13.20 & $\ldots$ & $\ldots$ & $\ldots$ & $\ldots$ & $\ldots$ \\
\hline 35 & 214309.85 & +660548.8 & M3 & 22.52 & 2.46 & 1.93 & 1.02 & 0.47 & 13.86 & $\ldots$ & $\ldots$ & $\ldots$ & $\ldots$ & $\ldots$ \\
\hline 36 & 214313.04 & +660550.8 & M4.5 & 20.49 & 1.62 & 1.84 & 0.80 & 0.39 & 13.64 & $\ldots$ & $\ldots$ & $\ldots$ & $\ldots$ & $\ldots$ \\
\hline 37 & 214314.63 & +661013.6 & K1 & 18.46 & 1.20 & 1.13 & 0.78 & 0.29 & 13.31 & 13.16 & 13.10 & $\ldots$ & $\ldots$ & $\ldots$ \\
\hline 38 & 214314.93 & +660907.1 & M0 & 17.99 & 1.05 & 0.92 & 0.66 & 0.26 & 13.86 & 13.73 & 13.80 & $\ldots$ & $\ldots$ & $\ldots$ \\
\hline 39 & $2143 \quad 17.55$ & +660418.5 & G0-2 & 20.29 & 1.51 & 1.42 & 0.89 & 0.47 & 13.58 & $\ldots$ & $\ldots$ & $\ldots$ & $\ldots$ & $\ldots$ \\
\hline 40 & 214320.84 & +6603 37.1 & M1 & 17.55 & 1.04 & 1.01 & 0.68 & 0.19 & 13.45 & 13.22 & 13.19 & $\ldots$ & $\ldots$ & $\ldots$ \\
\hline 41 & 214321.66 & +660246.1 & G3 & 14.25 & 0.44 & 0.42 & 0.40 & 0.03 & 12.40 & 12.32 & 12.34 & $\ldots$ & $\ldots$ & $\ldots$ \\
\hline 42 & 214331.12 & +660743.7 & M2 & 19.38 & 1.18 & 1.18 & 0.61 & -0.04 & 14.90 & $\ldots$ & 14.44 & $\ldots$ & $\ldots$ & $\ldots$ \\
\hline 43 & 214331.18 & +660724.4 & G8-K1 & 14.53 & 0.62 & 0.52 & 0.52 & 0.12 & 11.96 & 11.86 & 11.93 & $\ldots$ & $\ldots$ & $\ldots$ \\
\hline 44 & 214331.18 & +660954.7 & G6-8 & 15.70 & 1.33 & 1.24 & 0.93 & 0.32 & 9.96 & 9.74 & 9.72 & 9.85 & $\ldots$ & SVS 14 \\
\hline 45 & 214332.68 & +661011.8 & G-K1 & 20.16 & 1.49 & 1.38 & 1.19 & 0.25 & 13.55 & 13.15 & 13.15 & $\ldots$ & $\ldots$ & $\ldots$ \\
\hline 46 & 214340.01 & +660331.9 & G4 & 12.92 & 0.38 & 0.34 & 0.33 & 0.04 & 11.13 & 11.06 & 11.09 & 11.20 & $\ldots$ & $\ldots$ \\
\hline 47 & 214340.64 & +660516.5 & M5 & 21.50 & 1.38 & 1.80 & 0.56 & 0.76 & 15.08 & 15.14 & 15.05 & $\ldots$ & $\ldots$ & $\ldots$ \\
\hline
\end{tabular}


Table 3

(Continued)

\begin{tabular}{|c|c|c|c|c|c|c|c|c|c|c|c|c|c|c|}
\hline Source $^{a}$ & $\begin{array}{c}\alpha \\
(\mathrm{J} 2000)\end{array}$ & $\begin{array}{c}\delta \\
(\mathrm{J} 2000)\end{array}$ & $\mathrm{SpT}$ & $V^{\mathrm{b}}$ & $V-R_{C}^{\mathrm{b}}$ & $R_{C}-I_{C}^{\mathrm{b}}$ & $J-H^{\mathrm{c}}$ & $H-K_{S}^{\mathrm{c}}$ & $K_{S}^{\mathrm{c}}$ & $w 1^{\mathrm{d}}$ & $w 2^{\mathrm{d}}$ & $w 3^{\mathrm{d}}$ & $w 4^{\mathrm{d}}$ & Comments \\
\hline 48 & 214344.89 & +66070.12 & A2 & 15.29 & 0.90 & 0.90 & 0.49 & 0.21 & 11.40 & 11.17 & 11.13 & $\ldots$ & $\ldots$ & $\ldots$ \\
\hline 49 & 214345.10 & +660438.1 & G8 & 15.57 & 0.56 & 0.46 & 0.37 & 0.15 & 13.28 & 13.18 & 13.21 & $\ldots$ & $\ldots$ & $\ldots$ \\
\hline 50 & 214345.37 & +660822.9 & M1 & 17.50 & 1.09 & 1.10 & 0.58 & 0.28 & 13.22 & 12.83 & 12.62 & $\cdots$ & $\cdots$ & IR excess \\
\hline 51 & 214347.03 & +661001.7 & G5-K0 & 19.37 & 1.22 & 1.11 & 0.70 & 0.51 & 14.19 & 13.84 & 13.82 & $\ldots$ & $\ldots$ & $\ldots$ \\
\hline 52 & 214347.40 & +660849.5 & M3 & 20.01 & 1.16 & 1.26 & 0.81 & 0.48 & 15.05 & $\ldots$ & $\ldots$ & $\ldots$ & $\ldots$ & $\ldots$ \\
\hline 53 & 214350.02 & +660752.3 & M3.5 & 16.84 & 1.22 & 1.46 & 0.60 & 0.23 & 11.94 & 11.68 & 11.53 & $\ldots$ & $\ldots$ & $\ldots$ \\
\hline 54 & 214350.48 & +660938.8 & K2 & 18.72 & 1.29 & 1.22 & 0.90 & 0.30 & 13.11 & 12.69 & 12.74 & $\ldots$ & $\ldots$ & $\ldots$ \\
\hline 55 & 214351.63 & +660339.0 & K0-3 & 19.95 & 1.20 & 1.12 & 0.92 & 0.19 & 14.70 & 14.66 & 14.71 & $\ldots$ & $\ldots$ & $\ldots$ \\
\hline 56 & 214354.23 & +660642.5 & K5: & 21.18 & 1.66 & 1.37 & 1.14 & 0.23 & 14.37 & 13.91 & 13.91 & $\cdots$ & $\cdots$ & $\cdots$ \\
\hline 57 & 214354.30 & +660910.4 & K5: & 20.25 & 1.36 & 1.20 & 0.96 & 0.45 & 14.48 & 14.12 & 14.32 & $\cdots$ & $\cdots$ & $\cdots$ \\
\hline 58 & 214355.69 & +660529.6 & K5: & 19.63 & 1.46 & 1.33 & 0.98 & 0.37 & 13.29 & 12.99 & 12.97 & $\cdots$ & $\cdots$ & $\cdots$ \\
\hline 59 & 214355.58 & +660816.9 & F7-G5 & 18.54 & 1.11 & 1.03 & 0.72 & 0.19 & 13.96 & 13.66 & 13.69 & $\ldots$ & $\ldots$ & $\ldots$ \\
\hline 60 & 214355.98 & +660305.3 & G6 & 15.68 & 0.62 & 0.53 & 0.56 & 0.10 & 13.11 & 13.04 & 13.08 & $\cdots$ & $\cdots$ & $\cdots$ \\
\hline 61 & 214358.34 & +660711.7 & K5 & 21.26 & 1.72 & 1.29 & 0.91 & 0.45 & 14.73 & 14.55 & 14.56 & $\ldots$ & $\cdots$ & $\cdots$ \\
\hline 62 & 214359.96 & +660437.0 & $\mathrm{K} 4$ & 16.90 & 0.83 & 0.65 & 0.65 & -0.03 & 13.83 & 13.59 & 13.67 & $\cdots$ & $\cdots$ & $\cdots$ \\
\hline 63 & 214400.52 & +660517.3 & M0 & 16.82 & 1.00 & 0.95 & 0.74 & 0.14 & 12.85 & 12.64 & 12.63 & $\cdots$ & $\cdots$ & $\cdots$ \\
\hline 64 & 214400.95 & +660724.7 & $\mathrm{K} 1$ & 18.20 & 1.40 & 1.26 & 0.97 & 0.33 & 12.31 & 12.08 & 12.11 & $\ldots$ & $\ldots$ & $\ldots$ \\
\hline 65 & 214402.99 & +660456.2 & G-K & 19.20 & 1.39 & 1.23 & 0.89 & 0.31 & 13.46 & 13.20 & 13.19 & $\ldots$ & $\ldots$ & $\ldots$ \\
\hline 66 & 214404.32 & +660641.6 & $\mathrm{K} 2$ & 16.74 & 1.31 & 1.20 & 0.90 & 0.27 & 11.23 & 11.02 & 11.04 & $\ldots$ & $\ldots$ & $\ldots$ \\
\hline 67 & 214405.12 & +660600.2 & F7 & 17.04 & 1.03 & 0.96 & 0.61 & 0.21 & 12.78 & 12.63 & 12.58 & $\ldots$ & $\cdots$ & $\cdots$ \\
\hline 68 & 214405.41 & +660452.6 & M2 & 18.65 & 1.11 & 1.09 & 0.55 & 0.16 & 14.50 & 14.20 & 14.15 & $\cdots$ & $\cdots$ & $\cdots$ \\
\hline 69 & 214406.59 & +660913.1 & G7-K0 & 17.02 & 1.14 & 1.05 & 0.82 & 0.20 & 12.23 & 12.04 & 12.05 & $\ldots$ & $\ldots$ & $\ldots$ \\
\hline 70 & 214406.71 & +660810.0 & G5 & 17.84 & 0.96 & 0.90 & 0.56 & 0.19 & 14.01 & 13.87 & 13.85 & $\ldots$ & $\ldots$ & $\ldots$ \\
\hline 71 & 214408.14 & +660604.2 & G7-K0 & 18.51 & 1.13 & 1.02 & 0.80 & 0.11 & 13.88 & 13.44 & 13.38 & $\ldots$ & $\ldots$ & IR excess? \\
\hline 72 & 214408.64 & +660315.9 & G5-8 & 19.47 & 1.30 & 1.18 & 0.86 & 0.42 & 13.91 & 13.70 & 13.70 & $\cdots$ & $\cdots$ & $\ldots$ \\
\hline
\end{tabular}

Notes.

${ }^{a}$ Identifier.

${ }^{\mathrm{b}}$ Optical photometry from the KPNO $0.9 \mathrm{~m}$ or the UH $2.2 \mathrm{~m}$.

${ }^{\mathrm{c}}$ Near-infrared photometry from the 2MASS Point Source Catalog.

${ }^{\mathrm{d}}$ Mid-infrared photometry from the AllWISE Source Catalog. 

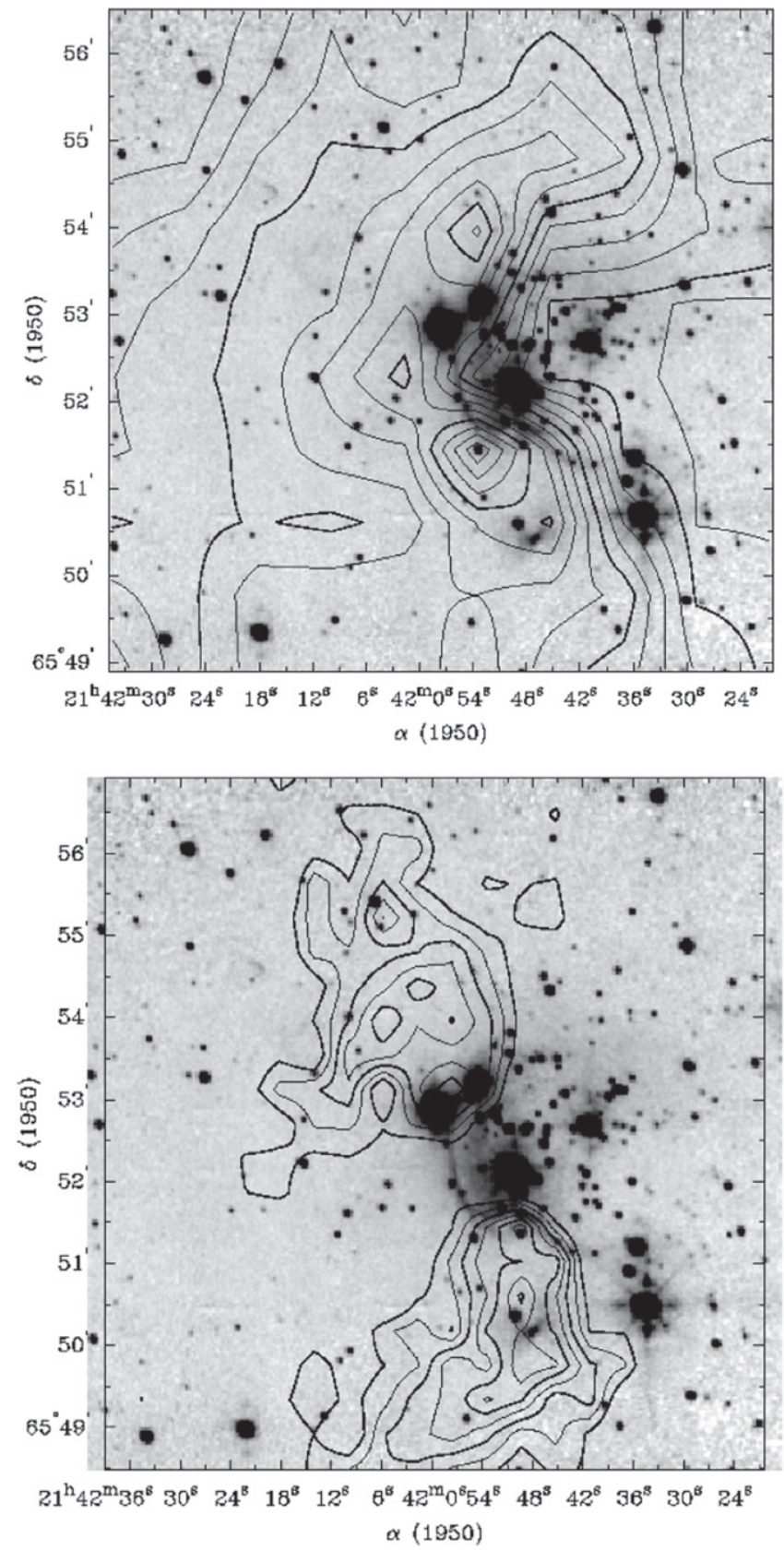

Figure 14. (a) Map of visual extinction $\left(A_{V}\right)$ derived from ${ }^{13} \mathrm{CO}$ integrated line intensity obtained at the Five College Radio Astronomical Observatory (FCRAO) in 1993 by LAH. The contour map is overlaid upon a near-infrared mosaic image of NGC 7129 obtained on the KPNO 50 inch telescope using the SQIID camera. Extinction contours are plotted with intervals of 5 mag over the range from 2.5 to $60 \mathrm{mag}$. (b) A map of integrated CS line intensity from data obtained at FCRAO superposed upon the same near-infrared mosaic image of NGC 7129. Extinction contours are plotted with intervals of $A_{V} \sim 0.25 \mathrm{mag}$ over the range from 1.0 to 2.5 mag.

cluster stars in the color-magnitude diagram. A collection of faint $(V>18 \mathrm{mag}) \mathrm{H} \alpha$ emission sources are evident outside of the nominal cluster sequence lying on or just below the ZAMS. Most of these sources lie on the periphery of the molecular clouds, three to the west where extinction is minimal. If taken at face value, the isochronal ages assigned to these sources would be much greater than that of the cluster itself. Such objects are generally interpreted as having edge-on disk geometries that dramatically reduce stellar luminosity, or as

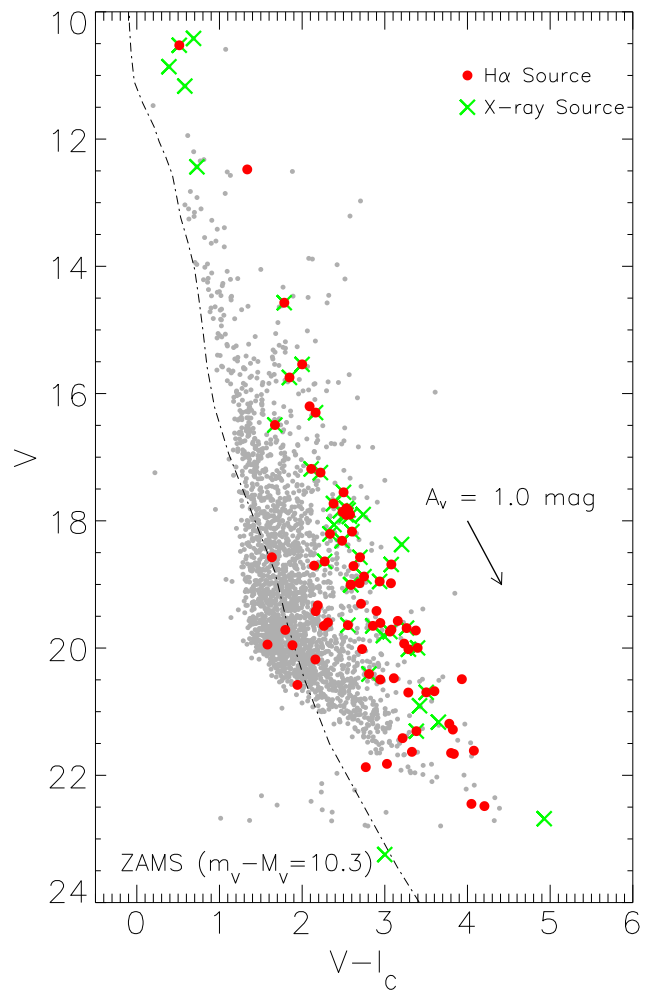

Figure 15. Observed $V-I_{C}, V$ color-magnitude diagram of NGC 7129 with the $\mathrm{H} \alpha$ emission sources shown as solid red circles, X-ray sources as green crosses, and other sources from the KPNO T2KA survey as gray points. The zero age main sequence (ZAMS) of Siess et al. (2000) is overplotted assuming the dwarf colors presented by Kenyon \& Hartmann (1995), derived from those of Bessell \& Brett (1988) and a distance of 1150 pc (Straižys et al. 2014).

background contaminants. The roughly $10 \%$ fraction of sources lying substantially below the cluster pre-main sequence and potentially having edge-on disk geometries is consistent with having a random distribution of disk orientations.

In Figure 16 we show the extinction-corrected $\left(V-I_{C}\right)_{0}, V_{0}$ color-magnitude diagram for the $\mathrm{H} \alpha$ emission stars and X-ray sources. Stars of known spectral type have been corrected individually for reddening using the intrinsic colors of 5-30 Myr pre-main sequence stars from Pecaut \& Mamajek (2013). All other sources have been dereddened using the mean extinction value derived for the $\mathrm{H} \alpha$ and X-ray samples.

\subsection{The Ages and Masses of the Pre-main Sequence Population}

The age of the cluster emerging from NGC7129 is undoubtedly young given the presence of one and possibly two Herbig Be stars $(\mathrm{LkH} \alpha$ 234, SVS 13) as well as the classical Be star $\mathrm{BD}+65^{\circ} 1637$. As will be discussed in Section 6 , the evolutionary timescale of the PDR enveloping $\mathrm{BD}+65^{\circ} 1638$ may be even younger, $<10^{4}$ year. Cluster ages are generally inferred by fitting their stellar populations in colormagnitude diagrams with pre-main sequence isochrones.

The grid of Siess et al. (2000) models was used to estimate the ages and masses for the optically detected $\mathrm{H} \alpha$ emitters and $\mathrm{X}$-ray sources having established spectral types. Uncertainties involved in the use of pre-main-sequence models fall into two broad categories; the physics used in modeling stellar evolution from the birthline to the zero-age main sequence, and the transformation between theoretical and observational planes. 


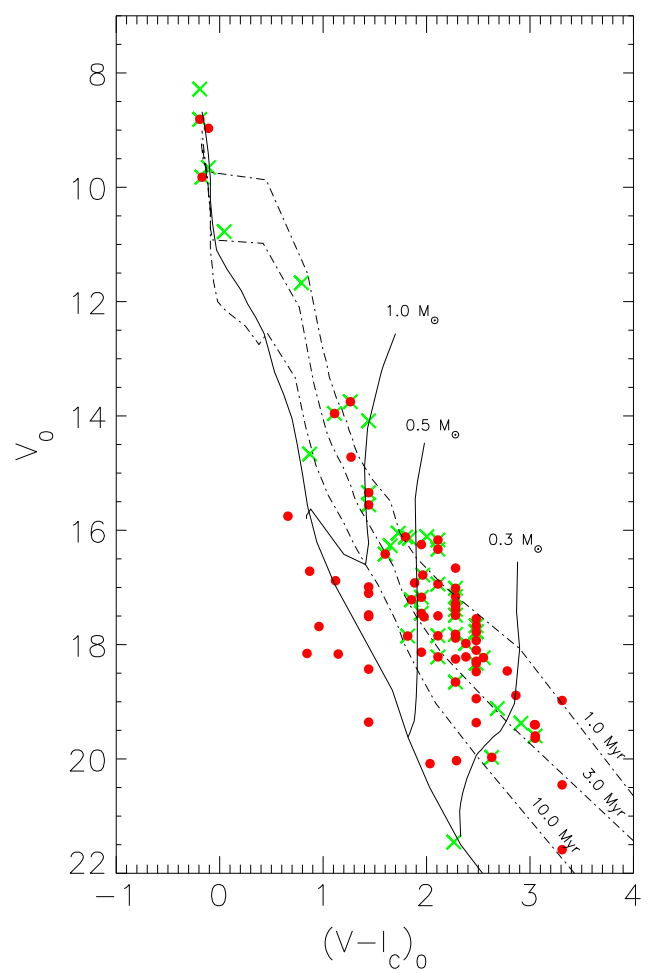

Figure 16. Extinction-corrected $\left(V-I_{C}\right)_{0}, V_{0}$ color-magnitude diagram for the $\mathrm{H} \alpha$ emission stars and X-ray sources with available optical photometry. Stars of known spectral type have been corrected individually for reddening using the intrinsic colors of 5-30 Myr pre-main sequence stars from Pecaut \& Mamajek (2013). Sources without spectral type information are plotted using the mean extinction derived for the cluster $A_{V}=1.79 \mathrm{mag}$. Superposed are the $0.5,1.0,3.0$, and $10 \mathrm{Myr}$ isochrones and the $0.3,0.5$, and $1.0 M_{\odot}$ evolutionary tracks of the solar metallicity models of Siess et al. (2000), assuming a distance of 1150 pc (Straižys et al. 2014).

Models of pre-main sequence evolution treat convection, opacity, radiative transfer, rotation, and accretion uniquely, leading to variations in predicted evolutionary paths for a given stellar mass. Initial conditions establishing the birthline are also not well understood. For an in depth discussion of issues related to the ages of young stars, the reader is referred to Soderblom et al. (2014).

Transforming between theoretical and observational planes is typically achieved by fitting main-sequence colors and bolometric corrections as a function of effective temperature, a problematic assumption given the lower surface gravities, cooler temperatures, enhanced chromospheric activity and accretion processes generally associated with pre-mainsequence stars. The Siess et al. (2000) models adopt the dwarf colors of Kenyon \& Hartmann (1995), which themselves are derived from Bessell \& Brett (1988).

With these caveats stated, the median age for the 50 cluster members having established spectral types determined from the models of Siess et al. (2000) is $\sim 1.8 \mathrm{Myr}$. The majority of sources in Figure 16 fall between the 1 and 3 Myr isochrones, implying relative coevality, within the tolerance of expected errors that are summarized by Soderblom et al. (2014). The spread in extinction-corrected, visual magnitude for a given color or effective temperature varies between one and two mag for $V-I_{C}>1.5$. A literal reading of the implied distribution of ages suggests a full range from 0.1 to $\sim 35 \mathrm{Myr}$; however some of the more advanced ages are potentially attributed to neutral extinction induced by edge-on disk geometries. These ages are

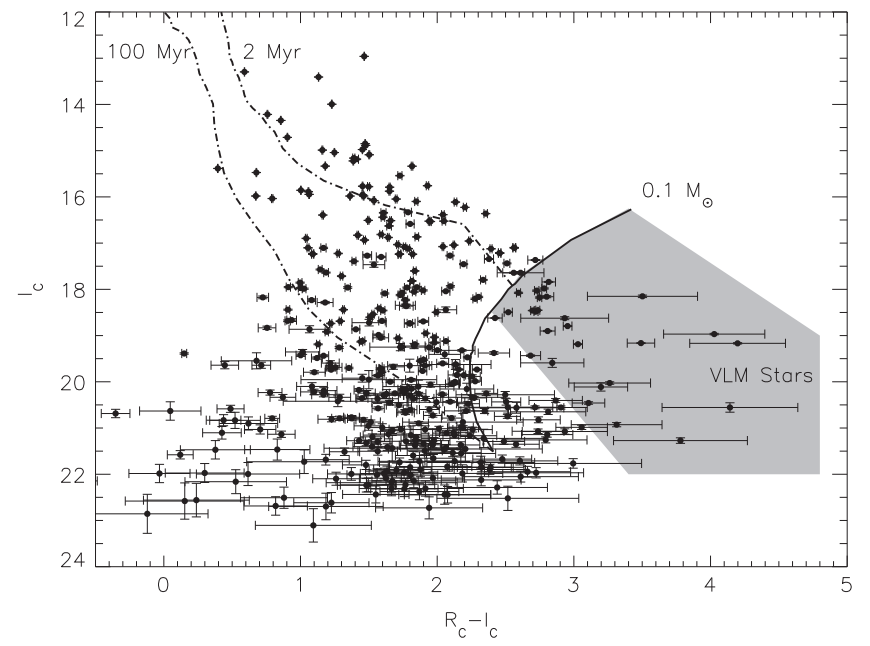

Figure 17. Observed $R_{C}-I_{C}, I_{C}$ color-magnitude diagram of NGC 7129 constructed from the Keck LRIS photometry, uncorrected for reddening. Photometric errors are shown for all sources. The Siess et al. (2000) isochrones for $2 \mathrm{Myr}$, the approximate cluster age, and $100 \mathrm{Myr}$ and the evolutionary track for a $0.1 M_{\odot}$ star are overplotted. The shaded region represents the area where very low mass cluster members are expected to lie, including brown dwarf candidates. The Baraffe et al. (1998) models predict that the sub-stellar mass limit lies near $I_{C}=19.83 \mathrm{mag}$, where a handful of sources are evident in the cluster sequence. Deeper photometric surveys as well as spectroscopy are needed for confirmation.

sensitive to the assumed distance of the cluster, intrinsic variability, errors in extinction, as well as unresolved binaries. The extreme case of having companions of equal mass would elevate stars in the color-magnitude diagram by $0.75 \mathrm{mag}$, resulting in the assignment of younger ages.

Recently, revisions to age estimates of young clusters and associations have advanced published ages by a factor of two or more. Pecaut et al. (2012) fit isochrones to the F and G-type stars in the color-magnitude diagram of the Upper Scorpius $\mathrm{OB}$ association, suggesting a revision to its age from $~ 5 \mathrm{Myr}$, which characterizes the $\mathrm{K}$ and M-type stellar population, to $11 \mathrm{Myr}$ if effects of the stellar birthline in the HR diagram (resulting from the finite radii of collapsing protostars) can be neglected. Bell et al. (2013) fit cluster populations with new semi-empirical isochrones which adopt empirically derived color-effective temperature relationships and bolometric corrections for pre-main sequence stars. The resulting ages of several young clusters and star-forming regions including IC 5146, IC 348, and NGC 2362 were subsequently adjusted by Bell et al. (2013) from $<1$ to $\sim 2 \mathrm{Myr}$, from $\sim 1.3$ to $\sim 6 \mathrm{Myr}$, and from $\sim 5$ to $\sim 12 \mathrm{Myr}$, respectively. Application of such techniques to the population of NGC 7129 would similarly advance its age from the estimate derived here if these techniques and assumptions are robust to further tests.

The inferred masses of the cluster members range from $\sim 0.2$ $M_{\odot}$ to $5.6 M_{\odot}$ (the most massive being $\mathrm{BD}+65^{\circ} 1638$ ), implying under standard initial mass function assumptions that a substantial fraction of low-mass stars and brown dwarfs remain unaccounted for in the activity-selected sample. Assuming a photometric completeness limit of $V \sim 21$ within the central cluster region implies that the survey is complete to about $\sim 0.3 M_{\odot}$. The Keck LRIS $R_{C}$ and $I_{C}$-band imaging is the deepest of the three photometric surveys presented here, extending to $I_{C} \sim 22$ within the core of NGC 7129 where extinction is significantly reduced. Shown in Figure 17 is the observed $R_{C}-I_{C}, I_{C}$ color-magnitude diagram for the Keck 

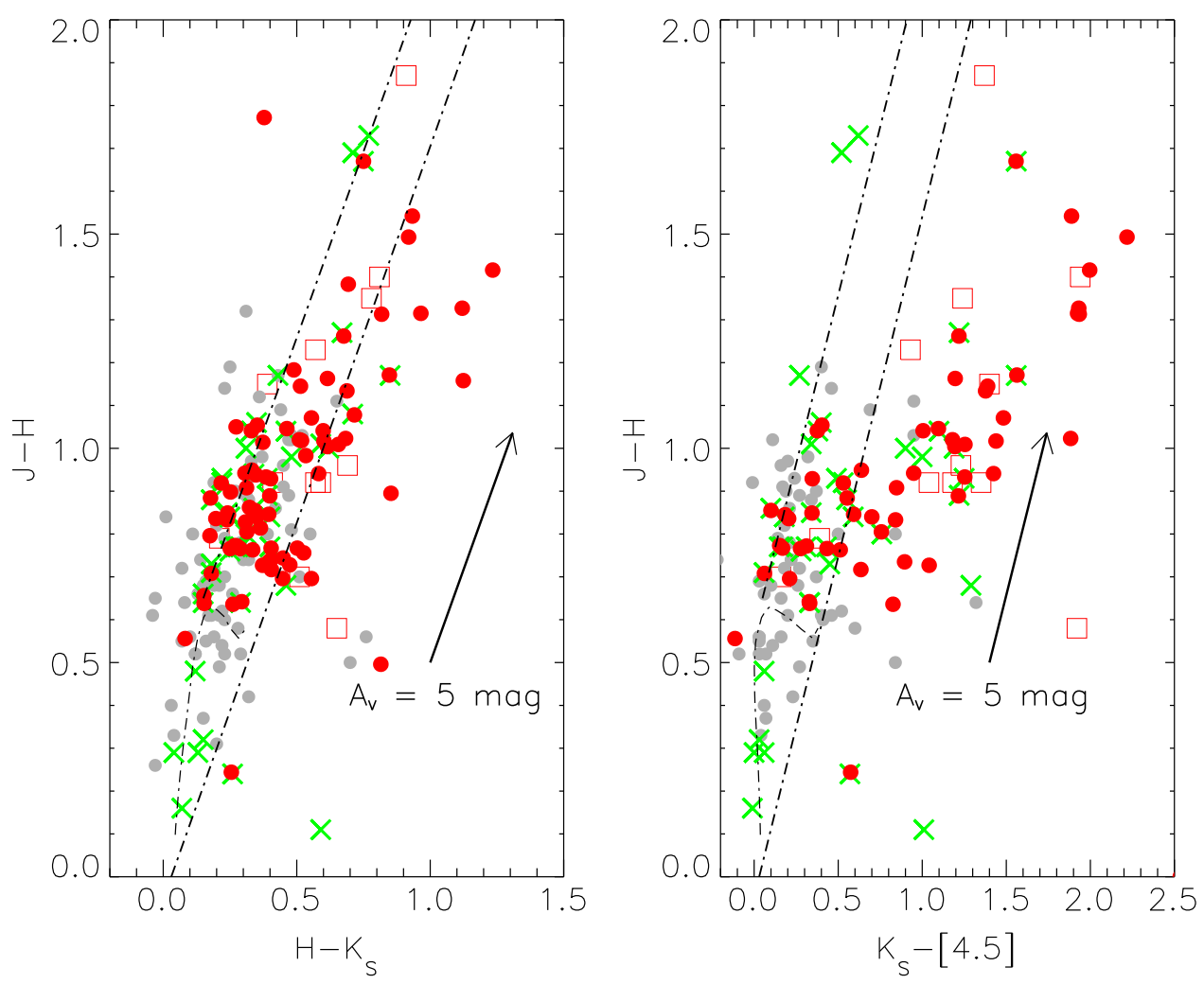

Figure 18. $H-K_{S}, J-H$ (left panel) and the $K_{S}-$ [4.5], $J-H$ (right panel) color-color diagrams for the $\mathrm{H} \alpha$ emission sources (red circles), X-ray sources (green crosses), infrared excess sources lacking X-ray emission or $\mathrm{H} \alpha$ emission (red squares) and spectroscopically classified stars lacking either $\mathrm{H} \alpha$ emission or X-ray emission (gray circles). The main sequence colors of Pecaut \& Mamajek (2013) are overplotted in both panels as dashed lines and the approximate reddening boundaries for dwarfs are shown with slopes derived using extinction data for diffuse interstellar clouds from Martin \& Whittet (1990). Assuming the extinction boundary for dwarfs in the $K_{S}-$ [4.5], $J-H$ color-color diagram to represent the demarcation line for disk-bearing sources, we find the disk fraction for all activityselected sources (i.e., $\mathrm{X}$-ray, $\mathrm{H} \alpha$ emission) included here to be $\sim 57 \% \pm 9 \%$.

LRIS photometry, uncorrected for reddening. The Siess et al. (2000) models do not extend below $0.1 M_{\odot}$, but it is clear from the figure that about two dozen sources are present that would lie along the $2 \mathrm{Myr}$ isochrone if extrapolated to lower masses. The evolutionary models of Baraffe et al. (1998) extend to much lower masses, well below the substellar mass limit and into the planetary mass regime. Assuming a mean extinction of $A_{V} \sim 1.8 \mathrm{mag}$ or $A_{I} \sim 1.0 \mathrm{mag}$, the Baraffe et al. (1998) models predict that the sub-stellar mass limit for NGC 7129 lies near $M_{I}=8.53$ or $I_{C}=19.83 \mathrm{mag}$ in Figure 17. Here there are a handful of sources in the photometric cluster sequence that are possible very low mass stars or brown dwarf candidates, but a deeper photometric survey as well as spectroscopy, particularly in the near-infrared, are needed for confirmation.

\subsection{The Infrared Color-Color Diagram for the Cluster Population}

NGC 7129 was observed from 3.6 to $24 \mu \mathrm{m}$ with Spitzer using IRAC and MIPS as part of the Spitzer Young Stellar Cluster Survey (AOR: 3655168 and 3663616). These observations are presented by Megeath et al. (2004) as well as Gutermuth et al. (2004). Stelzer \& Scholz (2009) adopted the photometry of Gutermuth et al. $(2004,2009)$ to derive a disk fraction for the Chandra X-ray and infrared-selected sources of $\sim 51 \% \pm 14 \%$. In a mass-limited, lightly extincted sub-sample $\left(A_{V}<5 \mathrm{mag}\right)$ of pre-main sequence stars, Stelzer \& Scholz (2009) derive a disk fraction of $\sim 33_{-19}^{+24} \%$. Although considered the least-biased sub-sample, it is also the least complete in terms of the number of included cluster members.

Here we combine 2MASS, Spitzer IRAC, and WISE photometry for the activity-selected sources in NGC 7129 to examine infrared excesses for candidate cluster members. Shown in Figure 18 are the $H-K_{S}, J-H$ (left panel) and the $K_{S}-$ [4.5], $J-H$ (right panel) color-color diagrams for the $\mathrm{H} \alpha$ emission sources, X-ray sources, infrared excess sources not identified with $\mathrm{X}$-ray or $\mathrm{H} \alpha$ emission, and the spectroscopically classified sources lacking either $\mathrm{H} \alpha$ or X-ray emission. If IRAC [4.5] channel photometry were not available, WISE $w 2$ photometry was substituted. The main sequence colors of Pecaut \& Mamajek (2013) are overplotted and the approximate reddening boundaries for dwarfs are shown with slopes derived using the extinction coefficients for diffuse interstellar clouds taken from Martin \& Whittet (1990). The 2MASS $H-K_{S}, J-H$ color-color digram distinguishes the most prominent infrared excess sources that lie to the right of the reddening boundary for normal dwarfs. These excesses arise from hot $>1000 \mathrm{~K}$ dust lying within the innermost (0.1-1 AU, depending upon stellar luminosity) regions of the circumstellar disks. The $K_{S}-$ [4.5], $J-H$ color-color diagram is much more effective at isolating disk-bearing sources given that the IRAC [4.5] and WISE $w 2$ photometry is sensitive to cooler dust temperatures.

Assuming the reddening boundary for late-type dwarfs in the $K_{S}-$ [4.5], $J-H$ color-color diagram to represent the demarcating limit for disk-bearing sources, we find the disk fraction for the activity-selected sample having [4.5] or $w 2$ 
photometry available to be $57 \% \pm 9 \%$, consistent with the disk fractions derived by Gutermuth et al. (2004), 54\% $\pm 14 \%$, and that of Stelzer \& Scholz (2009), 51\% $\pm 14 \%$. This should be considered as a lower limit for the sample examined here given that stars having weak excesses and lying within the color boundaries of normal stars have been excluded from the disk population. The estimated disk fraction in NGC 7129 is similar to those derived for the comparably aged star-forming regions IC 348, 50\% $\pm 6 \%$ (Lada et al. 2006) and Chamaeleon I, 50\% $\pm 6 \%$ (Luhman et al. 2008), but substantially larger than the disk fractions of the more evolved Upper Scorpius OB association, 19\% (Carpenter et al. 2006) and NGC 2362, 19\% (Dahm \& Hillenbrand 2007).

Figure 18 suggests that $\mathrm{H} \alpha$ emission is a better discriminant for identifying disk-bearing sources than X-ray emission. Longer exposure times with ACIS onboard Chandra would certainly have identified additional cluster members, but the effectiveness of simple ground-based, slitless grism or multiobject spectroscopic techniques at isolating active stars cannot be ignored. It should also be noted that a handful of spectroscopically classified sources lacking either $\mathrm{H} \alpha$ or $\mathrm{X}$ ray emission appear to have substantial infrared excess and are therefore candidate cluster members. These sources are identified as having infrared excess in the comments column of Table 3. The majority of the classified sources lacking activity indicators, however, appear to cluster along the upper extinction boundary for normal dwarfs where reddened giants might be expected to lie, supporting their non-membership status in NGC 7129. In summary, to conduct a population census of young star-forming regions, $\mathrm{H} \alpha$ emission, X-ray emission, and infrared excess are needed to ensure completeness.

\section{DISCUSSION}

\subsection{Star Formation in NGC 7129 and the 105:4+09:9 Molecular Cloud Complex}

Star formation is at an advanced stage within NGC 7129 with the recently formed massive stars $\mathrm{BD}+65^{\circ} 1638$ and $\mathrm{BD}$ $+65^{\circ} 1637$ dissociating and dispersing molecular material in the center of the molecular cloud. The exact sequence of massive star formation has been debated in the literature with Bechis et al. (1978) and Miskolczi et al. (2001) suggesting that BD $+65^{\circ} 1638$ collapsed initially and that its ultraviolet flux alone is largely responsible for clearing the elongated cavity.

Matthews et al. (2003), however, argue that winds from BD $+65^{\circ} 1637$ excavated the cavity given that $\mathrm{BD}+65^{\circ} 1637$ is the more luminous of the pair and that in projection, is more centrally located within the cleared area. Their moderateresolution $\left(1^{\prime}\right) 21 \mathrm{~cm} \mathrm{H}$ I survey of NGC 7129 revealed a $\sim 30^{\prime}$ diameter ring of emission apparently associated with the surface of the molecular cloud. $\mathrm{H}_{2}$ on the cloud surface has likely been dissociated by interstellar ultraviolet radiation, producing the observed ring. Within this ring, Matthews et al. (2003) note a bright knot of $\mathrm{H}$ I emission centered on $\mathrm{BD}+65^{\circ}$ 1638 that extends well beyond the confines of the molecular ridge. They suggest that the $\mathrm{H}_{\mathrm{I}}$ knot and $\mathrm{BD}+65^{\circ} 1638$ are seen in projection against the near surface of the molecular cloud, and are not lying within it. This would account for the observed radial velocity difference of $\sim 2 \mathrm{~km} \mathrm{~s}^{-1}$ between the $\mathrm{CO}$ and the $\mathrm{H}$ I emission. Matthews et al. (2003) postulate that BD $+65^{\circ}$ 1638 is the younger of the two stars having only formed within the last $1500-10^{4}$ years on the cloud periphery. This would also imply that $\mathrm{BD}+65^{\circ} 1638$ is among just a handful of "dissociating stars" known, massive stars caught in the process of emerging from their natal shells.

The HIRES spectra of $\mathrm{BD}+65^{\circ} 1637$ and $\mathrm{BD}+65^{\circ} 1638$ clearly demonstrate that $\mathrm{BD}+65^{\circ} 1637$ is the more active of the pair. Strong $\mathrm{H} \alpha$ emission is apparent in the former, presumably formed by recombination within the surrounding gaseous disk. Stellar winds are responsible for the blueshifted forbidden emission present in the spectrum. In contrast, the spectrum of $\mathrm{BD}+65^{\circ} 1638$ is unremarkable with little evidence to suggest the extreme youth required of it to be a dissociating star. In the infrared, strong excess emission characteristic of circumstellar material does not become apparent in the SED of $\mathrm{BD}+65^{\circ} 1638$ until $\sim 8 \mu \mathrm{m}$. The star falls on the locus of the main sequence in the near-infrared $K_{S}-$ [4.5], $J-H$ colorcolor diagram shown in Figure $18 . \mathrm{BD}+65^{\circ} 1637$ lies well to the right of the sequence suggestive of infrared excess. BD $+65^{\circ}$ 1637 and $\mathrm{BD}+65^{\circ} 1638$ lie near each other on the extinctioncorrected, color-magnitude diagram shown in Figure 16 and suffer comparable levels of optical extinction, $A_{V}=1.7$ and 2.1 mag, respectively.

The preponderance of spectroscopic evidence suggests that $\mathrm{BD}+65^{\circ} 1638$ is the more quiescent and therefore more evolved star of the pair. The origin of the bright knot of $\mathrm{H}$ I emission is unresolved, but may represent an expanding shell of dissociated molecular gas driven by the UV flux from $\mathrm{BD}+65^{\circ}$ 1637 and $\mathrm{BD}+65^{\circ} 1638$. The presence of an extensive PDR inferred from an arc of $\mathrm{H}_{2}$ emission some $170^{\prime \prime}$ long or $0.95 \mathrm{pc}$, was noted by Schultz et al. (1997) in their narrow-band, nearinfrared imaging survey of NGC 7129. This PDR appears to outline the molecular ridge and is thought to be illuminated by $\mathrm{UV}$ emission from $\mathrm{BD}+65^{\circ} 1638$. Regardless of formation order, these early-type stars have disrupted the star formation process within L1181, effectively dispersing molecular material within the cavity and possibly induced star formation throughout the molecular cloud.

Triggered star formation has been suggested for the central cluster region (e.g., Miskolczi et al. 2001), particularly along the dense molecular ridge where $\mathrm{LkH} \alpha 234$ and 2MASS J21430696+660641.7 are located. Maps of CO column density (e.g., Bechis et al. 1978; Miskolczi et al. 2001), reveal another dense core $\sim 1 \mathrm{pc}$ south of $\mathrm{BD}+65^{\circ} 1638$ where the intermediate-mass Class 0 protostar FIRS 2 is located. The mass of this source has been estimated by Fuente et al. (2014) to be $\sim 2-8$ $M_{\odot}$. Whether triggered by compression from the formation of $\mathrm{BD}+65^{\circ} 1637$ and $\mathrm{BD}+65^{\circ} 1638$ or by some other event is unknown. Surprisingly few $\mathrm{H} \alpha$ emission sources or embedded Class 0 and I sources are found in this region, possibly implying that star formation is in its earliest phase here.

The activity-selected sample of candidate members is nearly evenly divided between those concentrated within the cluster core, defined as lying within $2^{\prime}(\sim 0.7 \mathrm{pc})$ of $\mathrm{BD}+65^{\circ} 1638$, and a dispersed population spread throughout the molecular cloud complex. The majority of this dispersed population lies within the northeast quadrant of L1181, extending from V350 Cep and SVS 10 to south of GGD 35 (HH 235). This broad arc spans more than three parsecs in projection and is characterized by the presence of $\mathrm{HH}$ objects, shocked molecular hydrogen emission, and molecular outflows. Over three dozen $\mathrm{H} \alpha$ emission sources, X-ray sources and embedded protostars have been identified, suggesting that star formation is well underway 


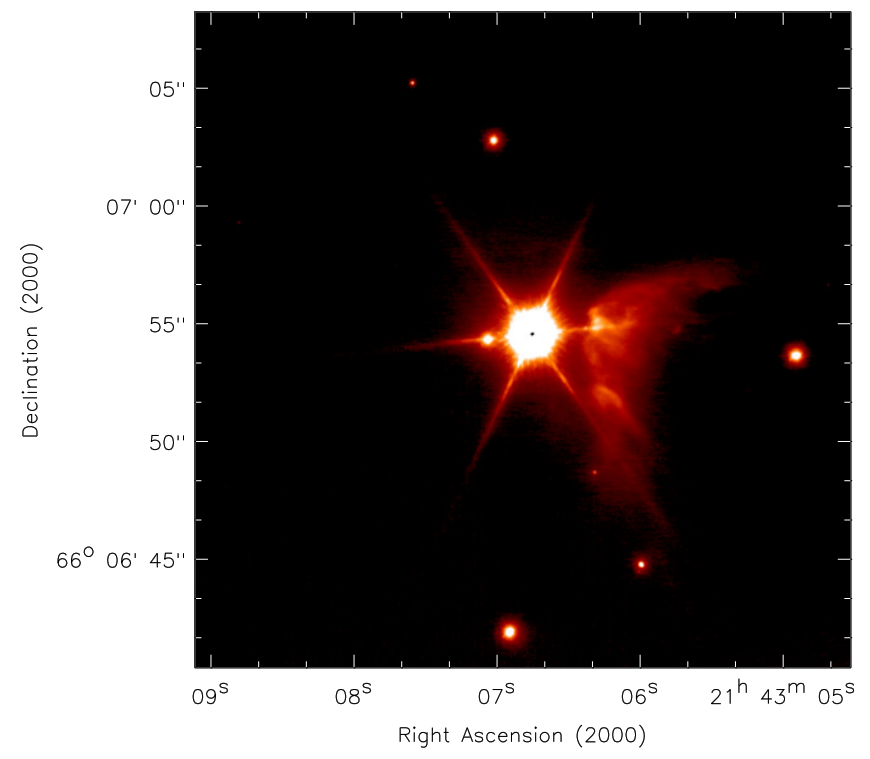

Figure 19. Median combined, $\sim 30^{\prime \prime} \times 30^{\prime \prime} K^{\prime}$ image centered near LkH $\alpha 234$ obtained using NIRC2 2 and the Keck II NGS AO system. The arc of nebulosity evident in the NIRC2 image represents the apex of the $\sim 170^{\prime \prime}$ long rim of $\mathrm{H}_{2}$ emission discussed by Schultz et al. (1997). A well-resolved companion of $\mathrm{LkH} \alpha 234$ having a PA of $97^{\circ}$ and a separation of 1.88 is evident as is the embedded young Class I source 2MASS J21430696+660641.7, some 12.5 to the south.

in this region of the molecular cloud. These isolated sources have likely formed independently of the central young cluster.

To the west, several $\mathrm{H} \alpha$ emitters are located around the molecular cloud core GGD 32, with HH 103 marking the terminus of star formation activity. No embedded protostars are found here and the detected $\mathrm{H} \alpha$ sources are predominantly knots of $\mathrm{HH}$ objects. The paucity of activity-selected sources further west suggests that few have drifted far from their formation sites, arguing for recent and rapid star formation in L1181.

\subsection{The Origin of the Molecular Outflow Near LkHo 234}

$\mathrm{LkH} \alpha 234$ lies at the apex of the one parsec scale, central cavity, the boundaries of which are demarcated on optical and infrared continuum images by bright arcs of nebulous emission and an increased density in stars, both cluster members and background sources. Northeast of NGC 7129, bright $4.5 \mu \mathrm{m}$ emission is evident in the Spitzer IRAC four-color image of the region presented by Gutermuth et al. (2004). This shocked CO emission is possibly produced by the impact of supersonic jets originating near $\mathrm{LkH} \alpha$ 234. $\mathrm{LkH} \alpha 234$ is also a radio continuum source (Bertout \& Thum 1982; Snell \& Bally 1986) and was thought to power an optical jet (Ray et al. 1990). The calculated mass loss rate for the optical outflow is $\sim 2-3 \times 10^{-8}$ $M_{\odot} \mathrm{yr}^{-1}$ and implies a momentum flux which is two orders of magnitude less than that of the molecular outflow. The optical jet points along the symmetry axis of the molecular cavity, with a number of faint stars aligned along the same axis. Because of its high collimation, however, the jet is unlikely to have formed the parsec-scale cavity. Ray et al. (1990) report that the $\mathrm{HH}$ objects present throughout the region are not associated with LkH $\alpha 234$ itself based upon a proper motion analysis. Shocked $\mathrm{H}_{2}$ emission was reported by Wilking et al. (1990) and Schultz et al. (1997) south of $\mathrm{LkH} \alpha 234$. This emission which could be related to the optical jet and to the $\mathrm{CO}$ molecular outflow is located on the opposite side of the star.

To investigate the source of the optical jet and the molecular outflow near $\mathrm{LkH} \alpha 234$, NIRC2 on Keck II imaged the region using its wide camera in $J, H$, and $K^{\prime}$. Shown in Figure 19 is a median combined, $\sim 30^{\prime \prime} \times 30^{\prime \prime} K^{\prime}$ image of the region having an effective integration time of $100 \mathrm{~s}$. The arc of nebulosity evident in the NIRC2 image represents the apex of the $\sim 170^{\prime \prime}$ long rim of $\mathrm{H}_{2}$ emission discussed by Schultz et al. (1997). $\mathrm{LkH} \alpha 234$ appears to be involved with this nebulosity, blowing back the gas and dust to form an illuminated wedgeshaped rim. The AO imaging reveals at least a dozen sources including a well-resolved companion of $\mathrm{LkH} \alpha 234$ having a position angle (PA) of $97^{\circ}$ and a separation of 1'.88. This source was noted previously by Perrin (2006) in near-infrared polarimetric observations of the region. About 12!.5 south of $\mathrm{LkH} \alpha 234$ lies the embedded Class I protostar 2MASS J21430696+660641.7, which only becomes prominent in the NIRC2 imaging at $K^{\prime}$.

The NIRC2 imaging also reveals two point sources along the southern arm of the nebulous arc, including a resolved visual binary with a separation of $\sim 0.33$ and $\Delta m_{K p} \sim 2.1$ mag. A luminous knot of nebular emission is apparent 2 !'7 distant from $\mathrm{LkH} \alpha$ 234, PA $280^{\circ}$. Weintraub et al. (1994) reported an embedded companion lying $3^{\prime \prime}$ northwest of $\mathrm{LkH} \alpha 234$ (PA $\left.290^{\circ}-340^{\circ}\right)$, their polarization source PS 1 , and suggested that this source is responsible for driving the bipolar molecular outflow associated with the region. PS 1 and their source IRS 5 $\left(\mathrm{PA}=330^{\circ}, 2^{\prime \prime} .5\right.$ separation from $\left.\mathrm{LkH} \alpha 234\right)$ roughly correspond with this bright knot of nebulous emission. The axis of the primary molecular outflow in the region is approximately $60^{\circ} / 240^{\circ}$, which corresponds with a substantial number of $\mathrm{HH}$ objects identified in the area (McGroarty et al. 2004).

Perrin (2006) describes two mid-infrared sources $\sim 3-4$ " northwest of $\mathrm{LkH} \alpha 234$, one triangular in shape and the other more point source-like. These sources are not detected in the NIRC2 imaging, but would be roughly coincident with PS 1 and the knot of nebulous emission. Reproduced in Figure 20 is an archival Spitzer IRAC [5.8] post basic calibrated data (BCD) image of the region, centered near $\mathrm{LkH} \alpha 234$. The PSF core of $\mathrm{LkH} \alpha 234$ is saturated in the image as is the Class I source 2MASS J21430696+660641.7. A bright infrared source corresponding to the mid-infrared sources of Perrin (2006) is evident as is a third point source $\sim 10^{\prime \prime}$ northwest of $\mathrm{LkH} \alpha 234$. These infrared sources appear to be roughly aligned with the bow-shocked nebular emission enshrouding SVS 13 further to the west. This bow-shocked emission either results from winds emanating from SVS 13 impacting surrounding molecular material or possibly from outflowing molecular gas from these embedded infrared sources colliding with winds from the earlytype star.

Between LkH $\alpha 234$ and 2MASS J21430696+660641 lies a third bright infrared point source in the IRAC [5.8] image that lacks counterpart in the NIRC2 $K^{\prime}$ image. This source is readily apparent in Spitzer IRAC [8.0] imaging and is likely another embedded Class 0 or I protostar. Assuming all NIRC2 and IRAC sources in this region to be cluster members, the stellar surface density in the immediate vicinity of $\mathrm{LkH} \alpha 234$ is several hundred $(>400)$ stars $\mathrm{pc}^{-2}$. Given the number of embedded sources emerging here, this region at the apex of the parsec-scale cavity must be extremely young. There are no 


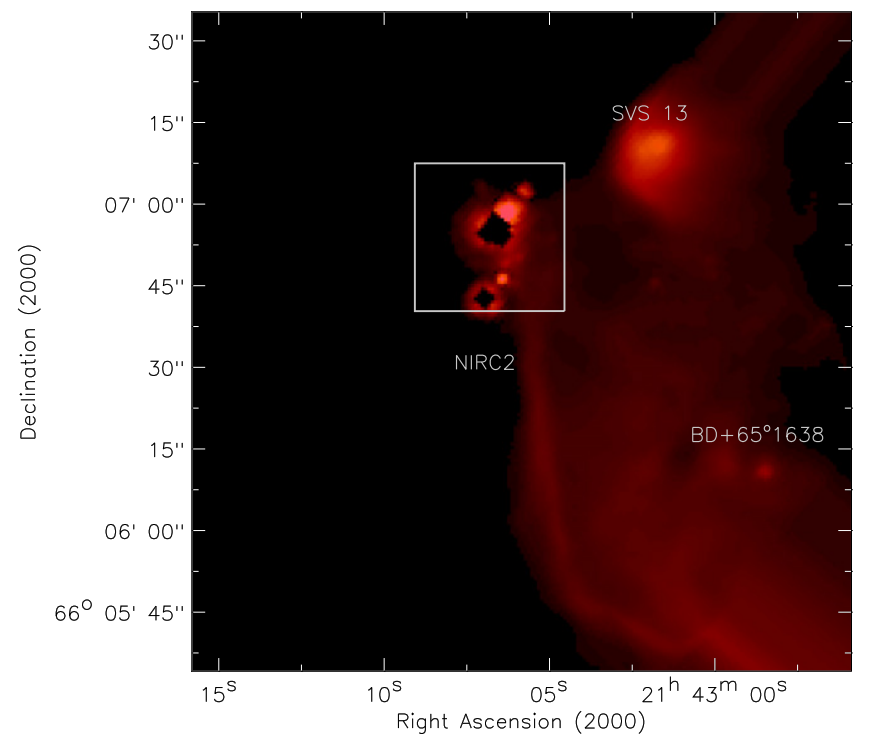

Figure 20. Spitzer IRAC [5.8] post BCD image centered near $\mathrm{LkH} \alpha 234$. For reference, the NIRC2 field of view is outlined in white. LkH $\alpha 234$ and 2MASS $\mathrm{J} 21430696+660641.7$ are saturated in the image, resulting in black cores for these sources. Three luminous infrared sources are evident that were not detected in the NIRC2 imaging of the region. It is possible that one of these sources is responsible for the molecular outflow and for the bow-shocked emission enveloping SVS 13 to the west.

other comparable examples of clustered, luminous protostars evident in the region.

\section{SUMMARY}

We have obtained deep, optical $\left(V, R_{C}, I_{C}\right)$ photometry for $\sim 2500$ sources in a field roughly centered on the reflection nebula NGC 7129 in Cepheus. Over $80 \mathrm{H} \alpha$ emission sources have been identified in the region, the majority of which are presumably members of a $\mathrm{T}$ Tauri star population emerging from the associated molecular cloud. Combined with 59 X-ray sources detected in a shallow, $22 \mathrm{ks}$ Chandra ACIS observation by Stelzer \& Scholz (2009), the $\mathrm{H} \alpha$ emission sources form a relatively narrow pre-main sequence in the $V-I_{C}, V$ colormagnitude diagram. Inspection of the color-magnitude diagram for all sources suggests that these two stellar activity indicators have identified the majority of the optically detectable pre-main sequence population, down to a limiting magnitude of $V \sim 20$.

Spectral types for over 130 sources in the region have been determined from low-dispersion, red optical spectroscopy. The mean extinction suffered by a sample of candidate cluster members is about $A_{V} \sim 1.8 \mathrm{mag}$. Using the pre-main sequence evolutionary models of Siess et al. (2000), we derive a median age of $\sim 1.8 \mathrm{Myr}$ for the $\mathrm{H} \alpha$ and X-ray selected sources having established spectral types, although a substantial age dispersion is present $(\sim 0.1-35 \mathrm{Myr})$.

Using near and mid-infrared photometry from 2MASS, Spitzer and WISE, we estimate the disk fraction of the activity- selected sample of cluster members to be $\sim 57 \% \pm 9 \%$. A small fraction of spectroscopically classified stars lacking either $\mathrm{H} \alpha$ or X-ray emission that exhibit infrared excess are present. We find a strong log-linear relationship between $W(\mathrm{H} \alpha)$ and $K_{S}-$ [4.5] color, as might be expected from these two inner disk indicators.

Merging the activity-selected sample of 94 sources with 31 infrared selected pre-main sequence candidates from Stelzer \& Scholz (2009) not detected with X-ray or $\mathrm{H} \alpha$ emission, and eight infrared excess sources from the spectroscopically classified sample of stars lacking X-ray or $\mathrm{H} \alpha$ emission, the cluster population of NGC 7129 has been increased from $\sim 90$ members to more than 130 .

High dispersion optical spectroscopy of BD+65 1638 and $\mathrm{BD}+65^{\circ} 1637$ obtained with HIRES on Keck I are presented which clearly demonstrate that $\mathrm{BD}+65^{\circ} 1637$ is the more active of the two, a probable classical Be star. The spectrum of BD $+65^{\circ} 1638$ is devoid of emission, suggesting the star to be remarkably quiescent if indeed a photodissociating star, just emerging from its primordial envelope of molecular gas.

HIRES spectroscopy of $\mathrm{LkH} \alpha 234$ is suggestive of active accretion with numerous metallic and forbidden transitions in emission as well as $\mathrm{H} \alpha$ and $\mathrm{H} \beta$. HIRES spectra obtained in 1999 and 2004 show significantly different $\mathrm{H} \alpha$ emission profiles including the presence of a deep absorption feature in the P Cygni-like structure in the earlier spectrum. The high dispersion spectrum of SVS 13 reveals weak emission reversal in the core of $\mathrm{H} \alpha$ as well as $\mathrm{Fe}_{\mathrm{I}} \lambda \lambda 4988,4994$ and [O I] $\lambda 6300$ emission. Three of four early-type stars within the cluster core are confirmed as Be stars.

Adaptive optics imaging of $\mathrm{LkH} \alpha 234$ using NIRC2 on Keck II reveals a number of embedded sources in the region including the Class I protostar 2MASS J21430696+660641.7. A bright, well-resolved companion of $\mathrm{LkH} \alpha 234$ is also detected having a PA of $97^{\circ}$ and a separation of 1 '!88. Of note several mid-infrared sources that are apparent in Spitzer IRAC and MIPS imaging of the region are not detected in the Keck AO $K^{\prime}$ imaging. Assuming all NIRC2 and IRAC sources to be associated with the cluster, the stellar surface density near $\mathrm{LkH} \alpha 234$ is several hundred stars $\mathrm{pc}^{-2}$.

Our interpretation of the structure of the remnant molecular clouds and the distribution of young stars in the region is that $\mathrm{BD}+65^{\circ} 1638$ is primarily responsible for evacuating the parsec-scale cavity in $\mathrm{L} 1181 . \mathrm{LkH} \alpha 234$ and a small number of deeply embedded protostars are forming within the ridge of compressed gas, possibly triggered by the collapse of $\mathrm{BD}+65^{\circ}$

Table 4

Optical Photometry from the T2KA CCD Imaging Survey of NGC 7129

\begin{tabular}{|c|c|c|c|c|c|c|c|}
\hline $\begin{array}{l}\text { R.A. } \\
\text { (h:m:s) }\end{array}$ & $\begin{array}{c}\text { Decl. } \\
\text { (d:m:s) }\end{array}$ & $\begin{array}{c}V \\
(\mathrm{mag})\end{array}$ & $\begin{array}{c}\operatorname{err}_{V} \\
(\mathrm{mag})\end{array}$ & $\begin{array}{c}\left(V-R_{C}\right) \\
(\mathrm{mag})\end{array}$ & $\begin{array}{c}\operatorname{err}_{\left(V-R_{C}\right)} \\
\quad(\mathrm{mag})\end{array}$ & $\begin{array}{c}\left(R_{C}-I_{C}\right) \\
\quad(\mathrm{mag})\end{array}$ & $\begin{array}{c}\operatorname{err}_{\left(R_{C}-I_{C}\right)} \\
\quad(\mathrm{mag})\end{array}$ \\
\hline $21: 41: 31.19$ & $65: 54: 3.8$ & 19.551 & 0.056 & 0.521 & 0.136 & 0.908 & 0.142 \\
\hline $21: 41: 31.71$ & $65: 54: 15.6$ & 21.532 & 0.149 & 1.381 & 0.165 & 1.702 & 0.082 \\
\hline $21: 41: 31.78$ & $65: 54: 26.8$ & 18.853 & 0.028 & 1.084 & 0.035 & 1.183 & 0.027 \\
\hline $21: 41: 32.54$ & $65: 54: 31.2$ & 20.015 & 0.048 & 0.780 & 0.061 & 0.811 & 0.061 \\
\hline $21: 41: 32.66$ & $65: 54: 51.0$ & 16.788 & 0.027 & 0.683 & 0.035 & 0.717 & 0.028 \\
\hline
\end{tabular}

(This table is available in its entirety in machine-readable and Virtual Observatory (VO) forms.) 
1638 and $\mathrm{BD}+65^{\circ} 1637$. Star formation within the compact cluster has been rapid and coeval given the relatively narrow pre-main sequence evident in the color-magnitude diagram. The formation of other intermediate-mass stars to the north and south of the cluster core including SVS 10 and FIRS 2, will likely further disrupt the molecular cloud, effectively ending the era of star formation in the region.

This paper is dedicated to the memory of George H. Herbig who passed away on 2013 October 12 at the age of 93 after a career spanning more than 70 years. He is considered by many to be the father of observational star formation, and as a testament to the impact of his contributions upon the field, classes of pre-main sequence objects now bear his name. An intellectual giant, his extraordinary physical insight, natural curiosity, and uncanny ability to ask the right questions led to tremendous advances in our understanding of the star formation process. There are few papers today on the subject of star formation that cannot trace their origins back to work initiated by George during his time at Lick Observatory, the University of California at Santa Cruz, and at the Institute for Astronomy, University of Hawaii.

We have made use of the Digitized Sky Surveys, which were produced at the Space Telescope Science Institute under U.S. Government grant NAG W-2166, the SIMBAD database operated at CDS, Strasbourg, France, and the 2MASS, a joint project of the University of Massachusetts and the Infrared Processing and Analysis Center (IPAC)/California Institute of Technology, funded by NASA and the National Science Foundation. This publication makes use of data products from the Wide-field Infrared Survey Explorer, which is a joint project of the University of California, Los Angeles, and the Jet Propulsion Laboratory/ California Institute of Technology, funded by the National Aeronautics and Space Administration. This research has made use of the Keck Observatory Archive, which is operated by the W. M. Keck Observatory and the NASA Exoplanet Science Institute (NExScI), under contract with the National Aeronautics and Space Administration. We thank Sirin Caliskan for early work on the LRIS data, performed as an undergraduate at Caltech and the referee whose suggestions improved the quality of the manuscript.

Facilities: Keck:I, Keck:II, Spitzer, Chandra, FCRAO, $\mathrm{UH}: 2.2 \mathrm{~m}$, WIYN, Mayall

\section{APPENDIX}

The complete list of all $\sim 2500$ sources from the T2KA CCD imaging survey of NGC 7129 including J2000 coordinates, optical $\left(V, V-R_{C}\right.$, and $\left.R_{C}-I_{C}\right)$ photometry, and associated photometric errors is provided in Table 4.

\section{REFERENCES}

Ábrahám, P., Leinert, C., Burkert, A., Henning, T., \& Lemke, D. 2000, A\&A, 354,965

Alecian, E., Wade, G. A., Catala, C., et al. 2013, MNRAS, 429, 1001

Allen, L. E., \& Strom, K. M. 1995, AJ, 109, 1379

Baraffe, I., Chabrier, G., Allard, F., \& Hauschildt, P. H. 1998, A\&A, 337, 403

Bechis, K. P., Harvey, P. M., Campbell, M. F., \& Hoffmann, W. F. 1978, ApJ, 226, 439

Bell, C. P. M., Naylor, T., Mayne, N. J., Jeffries, R. D., \& Littlefair, S. P. 2013, MNRAS, 434, 806

Bertout, C. 1987, Circumstellar Matter, 122, 23

Bertout, C. 1989, ARA\&A, 27, 351
Bertout, C., \& Thum, C. 1982, A\&A, 107, 368

Bessell, M. S., \& Brett, J. M. 1988, PASP, 100, 1134

Carpenter, J. M., Mamajek, E. E., Hillenbrand, L. A., \& Meyer, M. R. 2006, ApJ, 651, L49

Cohen, M., \& Fuller, G. A. 1985, ApJ, 296, 620

Cohen, M., \& Schwartz, R. D. 1983, ApJ, 265, 877

Courtois, H. M., Tully, R. B., \& Héraudeau, P. 2011, MNRAS, 415, 1935

Dahm, S. E. 2005, AJ, 130, 1805

Dahm, S. E., Herbig, G. H., \& Bowler, B. P. 2012, AJ, 143, 3

Dahm, S. E., \& Hillenbrand, L. A. 2007, AJ, 133, 2072

Dahm, S. E., \& Simon, T. 2005, AJ, 129, 829

Edwards, S., \& Snell, R. L. 1983, ApJ, 270, 605

Finkenzeller, U. 1985, A\&A, 151, 340

Finkenzeller, U., \& Jankovics, I. 1984, A\&AS, 57, 285

Fuente, A., Cernicharo, J., Caselli, P., et al. 2014, arXiv:1405.4639

Goodrich, R. W. 1986, AJ, 92, 885

Gutermuth, R. A., Megeath, S. T., Muzerolle, J., et al. 2004, ApJS, 154, 374 Gutermuth, R. A., Myers, P. C., Megeath, S. T., et al. 2008, ApJ, 674, 336

Hartigan, P., \& Lada, C. J. 1985, ApJS, 59, 383

Hawley, S. L., Gizis, J. E., \& Reid, I. N. 1996, AJ, 112, 2799

He, L., Whittet, D. C. B., Kilkenny, D., \& Spencer Jones, J. H. 1995, ApJS, 101, 335

Herbig, G. H. 1960, ApJS, 4, 337

Herbig, G. H. 1998, AJ, 497, 736

Herbig, G. H. 2008, AJ, 135, 637

Herbig, G. H., Andrews, S. M., \& Dahm, S. E. 2004, AJ, 128, 1233

Herbig, G. H., \& Dahm, S. E. 2002, AJ, 123, 304

Herbig, G. H., \& Dahm, S. E. 2006, AJ, 131, 1530

Hernández, J., Calvet, N., Briceño, C., Hartmann, L., \& Berlind, P. 2004, AJ, 127,1682

Hillenbrand, L. A. 1997, AJ, 113, 1733

Hillenbrand, L. A., Strom, S. E., Vrba, F. J., \& Keene, J. 1992, ApJ, 397, 613

Hodgkin, S. T., Jameson, R. F., \& Steele, I. A. 1995, MNRAS, 274, 869

Hubble, E. P. 1922, ApJ, 56, 400

Ibryamov, S., Semkov, E., \& Peneva, S. 2014, arXiv:1404.5902

Kenyon, S. J., \& Hartmann, L. 1995, ApJS, 101, 117

Kirkpatrick, J. D., Kelly, D. M., Rieke, G. H., et al. 1993, ApJ, 402, 643

Kun, M., Kiss, Z. T., \& Balog, Z. 2008, in Handbook of Star Forming Regions, Vol. 1, ed. B. Reipurth (San Francisco, CA: ASP), 136

Lada, C. J., Muench, A. A., Luhman, K. L., et al. 2006, AJ, 131, 1574

Landolt, A. U. 1992, AJ, 104, 340

Liseau, R., \& Sandell, G. 1983, RMxAA, 7, 199

Loren, R. B. 1975, Ph.D. thesis, University of Texas, Austin

Loren, R. B. 1977, ApJ, 218, 716

Lorenzetti, D., Giannini, T., Nisini, B., et al. 2003, in Exploiting the ISO Data Archive. Infrared Astronomy in the Internet Age, ed. C. Gry et al. (ESA SP511; Noordwijk: ESA), 137

Luhman, K. L., Allen, L. E., Allen, P. R., et al. 2008, ApJ, 675, 1375

Magakyan, T. Y. 1983, SvAL, 9, 83

Magakian, T. Y., Movsessian, T. A., \& Nikogossian, E. H. 2004, Ap, 47, 519

Magakyan, T. Y., \& Amirkhanyan, A. S. 1979, ATsir, 1038, 6

Martín, E. L. 1998, AJ, 115, 351

Martin, P. G., \& Whittet, D. C. B. 1990, ApJ, 357, 113

Matthews, H. E., Purton, C. R., Roger, R. S., Dewdney, P. E., \& Mitchell, G. F. 2003, ApJ, 592, 176

McGroarty, F., Ray, T. P., \& Bally, J. 2004, A\&A, 415, 189

Megeath, S. T., Allen, L. E., Gutermuth, R. A., et al. 2004, ApJS, 154, 367

Merrill, P. W., \& Burwell, C. G. 1950, ApJ, 112, 72

Miskolczi, B., Tothill, N. F. H., Mitchell, G. F., \& Matthews, H. E. 2001, ApJ, 560,841

Münch, G. 1957, ApJ, 125, 42

Muzerolle, J., Megeath, S. T., Gutermuth, R. A., et al. 2004, ApJS, 154, 379

Palla, F. 2005, in IAU Symp. 227, Massive Star Birth: A Crossroads of Astrophysics, ed. R. Cesaroni et al. (Cambridge: Cambridge Univ. Press), 196

Palla, F., \& Stahler, S. W. 1993, ApJ, 418, 414

Pecaut, M. J., Mamajek, E. E., \& Bubar, E. J. 2012, ApJ, 746, 154

Pecaut, M. J., \& Mamajek, E. E. 2013, ApJS, 208, 9

Perrin, M. D. 2006, Ph.D. thesis, University of California, Berkeley

Pogosyants, A. Y. 1991, IBVS, 3624, 1

Racine, R. 1968, AJ, 73, 233

Ray, T. P., Poetzel, R., Solf, J., \& Mundt, R. 1990, ApJ, 357, L45

Reid, N., Hawley, S. L., \& Mateo, M. 1995, MNRAS, 272, 828

Schultz, A. S. B., Rank, D. M., Temi, P., \& Holbrook, J. C. 1997, in IAU Symp. 182, Herbig-Haro Flows and the Birth of Stars ed. F. Malbet \& A. Castets, 39 
Shevchenko, V. S., \& Yakubov, S. D. 1989, AZh, 66, 718

Siess, L., Dufour, E., \& Forestini, M. 2000, A\&A, 358, 593

Slesnick, C. L., Carpenter, J. M., \& Hillenbrand, L. A. 2006, AJ, 131,3016

Snell, R. L., \& Bally, J. 1986, ApJ, 303, 683

Soderblom, D. R., Hillenbrand, L. A., Jeffries, R. D., Mamajek, E. E., \& Naylor, T. 2014, in Protostars and Planets VI, ed. H. Beuther et al. (Tucson, AZ: Univ. Arizona Press), 219

Stelzer, B., \& Scholz, A. 2009, A\&A, 507, 227

Straižys, V., Maskoliūnas, M., Boyle, R. P., et al. 2014, MNRAS, 438, 1848
Strom, S. E., Strom, K. M., Yost, J., Carrasco, L., \& Grasdalen, G. 1972, ApJ, 173,353

Strom, S. E., Vrba, F. J., \& Strom, K. M. 1976, AJ, 81, 638

Taylor, D. K., Dickman, R. L., \& Scoville, N. Z. 1987, ApJ, 315, 104

Vogt, S. S., et al. 1994, Proc. SPIE, 2198, 362

Weintraub, D. A., Kastner, J. H., \& Mahesh, A. 1994, ApJL, 420, L87

White, R. J., \& Basri, G. 2003, ApJ, 582, 1109

Wilking, B. A., Schwartz, R. D., Mundy, L. G., \& Schultz, A. S. B. 1990, AJ, 99, 344

Yonekura, Y., Dobashi, K., Mizuno, A., Ogawa, H., \& Fukui, Y. 1997, ApJS, 110,21 